Florida International University FIU Digital Commons

$7-1-2016$

\title{
Analysis of Improvised Explosives by Electrospray Ionization - Mass Spectrometry and Microfluidic Techniques
}

Inge Corbin

icorb001@fiu.edu

DOI: 10.25148 /etd.FIDC000771

Follow this and additional works at: https://digitalcommons.fiu.edu/etd

Part of the Analytical Chemistry Commons

\section{Recommended Citation}

Corbin, Inge, "Analysis of Improvised Explosives by Electrospray Ionization - Mass Spectrometry and Microfluidic Techniques" (2016). FIU Electronic Theses and Dissertations. 2551.

https://digitalcommons.fiu.edu/etd/2551 


\title{
FLORIDA INTERNATIONAL UNIVERSITY
}

Miami, Florida

\section{ANALYSIS OF IMPROVISED EXPLOSIVES BY ELECTROSPRAY IONIZATION - MASS SPECTROMETRY AND MICROFLUIDIC TECHNIQUES}

\author{
A dissertation submitted in partial fulfillment of \\ the requirements for the degree of \\ DOCTOR OF PHILOSOPHY \\ in \\ CHEMISTRY \\ by \\ Inge Corbin
}

2016 
To: Dean Michael R. Heithaus

College of Arts, Sciences and Education

This dissertation, written by Inge Corbin, and entitled Analysis of Improvised Explosives by Electrospray Ionization - Mass Spectrometry and Microfluidic Techniques, having been approved in respect to style and intellectual content, is referred to you for judgment.

We have read this dissertation and recommend that it be approved.

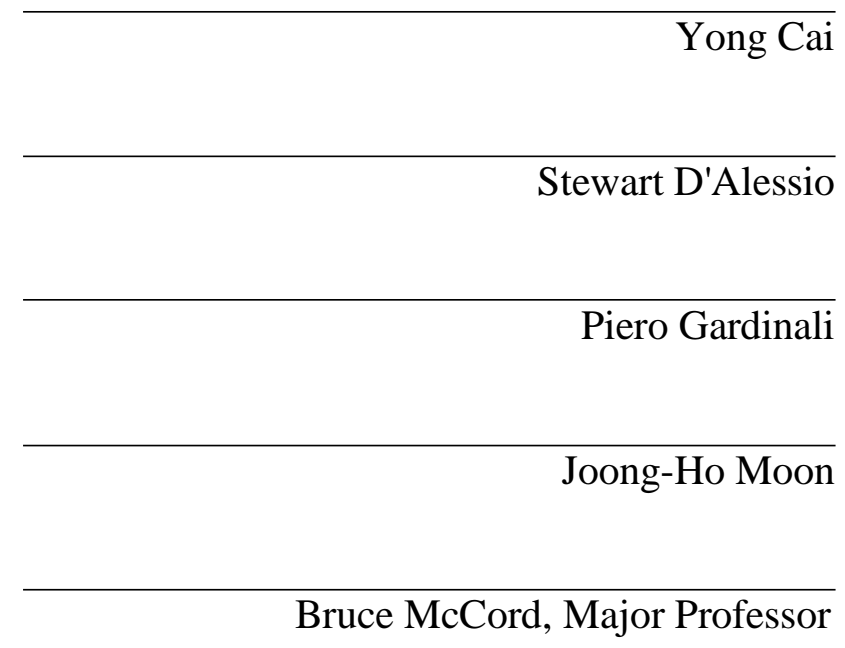

Date of Defense: July 1, 2016

The dissertation of Inge Corbin is approved.

Dean Michael R. Heithaus College of Arts, Sciences and Education

Andrés G. Gil Vice President for Research and Economic Development and Dean of the University Graduate School

Florida International University, 2016 
(C) Copyright 2016 by Inge Corbin

All rights reserved. 


\section{DEDICATION}

This work is dedicated to my husband, Larry McAllister. Never in a million years could I have done it without you.

IMO Juan, Helium, and Lifer. 


\section{ACKNOWLEDGMENTS}

I want to thank my major professor, Dr. Bruce McCord, and the members of my committee: Dr. Yong Cai, Dr. Stewart D’Alessio, Dr. Piero Gardinali, and Dr. Joong Ho Moon. Their patience and good advice throughout my graduate career is very much appreciated. I also want to thank Dr. Watson Lees, FIU Chemistry department's Graduate Program Director, for helping me with all the required paperwork while I was so far away.

I want to acknowledge the love and support of my parents, my sister Ursula, and my brother (in law) Brian. I also want to thank my sister from another mother, Christine Kruse. Whenever I lost my sense of humor all of you helped me find it again and carry on.

Finally, I would like to express my gratitude to the US Department of Homeland Security for their funding of grant number HSHQDC-09-C-00099, Analysis of Ammonium Nitrate and Urea Nitrate on Surfaces, and the US Department of Justice for their funding of contract number NIJ2012-DN-BX-K048, Development of Paper Microfluidic Devices for the On-Site Detection of Improvised Explosives. 


\title{
ABSTRACT OF THE DISSERTATION \\ ANALYSIS OF IMPROVISED EXPLOSIVES BY ELECTROSPRAY IONIZATION - MASS SPECTROMETRY AND MICROFLUIDIC TECHNIQUES
}

\author{
by \\ Inge Corbin
}

Florida International University, 2016

Miami, Florida

\section{Professor Bruce McCord, Major Professor}

Improvised explosives may be based on smokeless gunpowder, fertilizers, or inorganic oxidizers such as nitrate $\left(\mathrm{NO}_{3}{ }^{-}\right)$, chlorate $\left(\mathrm{ClO}_{3}{ }^{-}\right)$, and perchlorate $\left(\mathrm{ClO}_{4}{ }^{-}\right)$salts. Identification is a priority for the military and law enforcement but due to their varying physical properties and complexity, identification can be challenging. Consequently, three methods have been developed to aid in presumptive and confirmatory detection.

Smokeless powder contains plasticizers, stabilizers, dyes, opacifiers, flash suppressants, and other compounds. Identification of these additives can narrow down or identify the brands of smokeless powder used in a device. Fourteen organic smokeless powder components were identified by capillary electrochromatography (CEC) using a hexyl acrylate monolithic stationary phase coupled to UV detection and time-of-flight mass spectrometry (TOF-MS). The CEC-UV method efficiently detects all 14 organic components, while TOF-MS provides sensitivity and selectivity. A mixed smokeless powder component standard was analyzed and the composition of the additive package in commercial smokeless powders determined. Detection limits ranged from $1.0-3.2 \mu \mathrm{g} / \mathrm{ml}$ and analysis time was 18 minutes. 
Second, a procedure for the detection of urea nitrate (UN) and ammonium nitrate (AN) by infusion electrospray ionization - mass spectrometry (ESI-MS/MS) was developed. Solubility tests were performed to find a solvent for both UN and AN that did not cause UN to dissociate. Two adduct ions were detected for each explosive: for AN, m/z 178 $\left[2 \mathrm{AN}+\mathrm{NH}_{4}\right]^{+}$and $\mathrm{m} / \mathrm{z} 258\left[3 \mathrm{AN}+\mathrm{NH}_{4}\right]^{+}$ions, and for $\mathrm{UN} \mathrm{m} / \mathrm{z} 185\left[\mathrm{UN}+\mathrm{NO}_{3}\right]^{-}$and $\mathrm{m} / \mathrm{z}$ $248\left[\mathrm{UN}+\mathrm{HNO}_{3}+\mathrm{NO}_{3}\right]^{-}$. Specificity of the analysis was examined by mixing the explosives with various salts and interferents. Gas-phase adduct ions were useful in distinguishing between ion pairs and mixed salts.

Finally, a paper microfluidic device (PMD) was developed as a presumptive test using colorimetric reagents for the detection of ions associated with improvised explosives. The device was configured to test for nitrate $\left(\mathrm{NO}_{3}{ }^{-}\right)$, nitrite $\left(\mathrm{NO}_{2}{ }^{-}\right)$, chlorate $\left(\mathrm{ClO}_{3}{ }^{-}\right)$, perchlorate $\left(\mathrm{ClO}_{4}{ }^{-}\right)$, and urea nitrate (UN). Proof of concept was performed using extracts of soil containing inorganic oxidizers.

The development of these analytical methods allows the detection of smokeless powder components, fertilizers, and oxidizers and expands the suite of analytical methods available for the analysis of improvised explosives. 


\section{TABLE OF CONTENTS}

I. CHAPTER 1: A Short History of Improvised Explosives and Devices ................................... 1

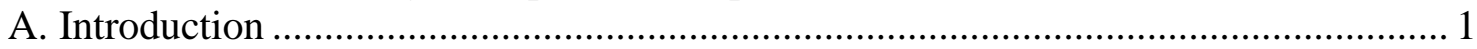

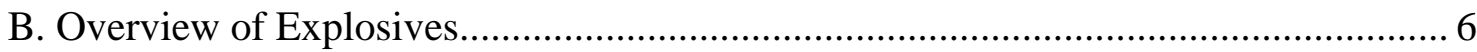

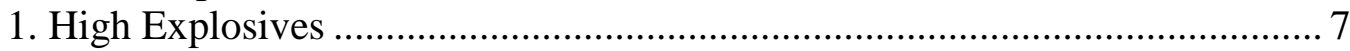

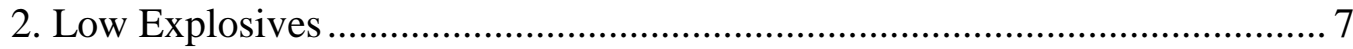

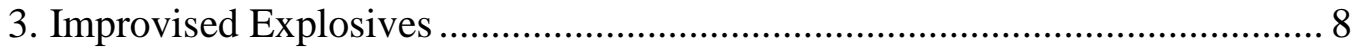

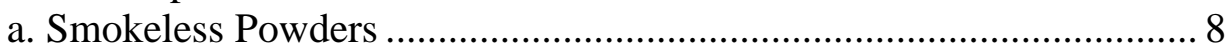

b. Ammonium Nitrate and Urea Nitrate............................................ 10

c. Improvised Explosives and Pyrotechnic Compositions ...................... 12

C. Forensic Applications of Explosives Analysis .......................................................... 13

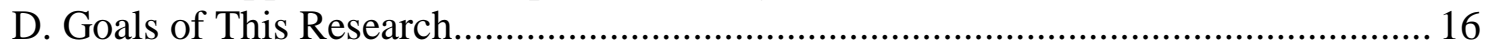

II. CHAPTER 2: Separation and Identification of Organic Components of Smokeless Gunpowder by Capillary Electrochromatography ………...................................................... 23

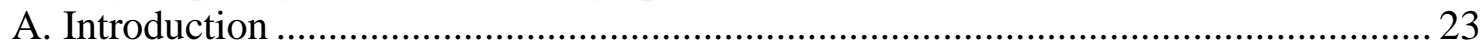

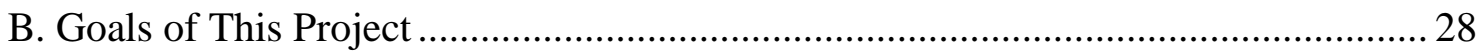

C. Fundamentals of Capillary Electrochromatography ................................................. 29

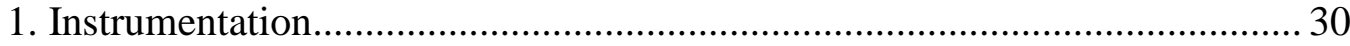

a. Electroosmotic Flow (EOF) ………………………………..... 30

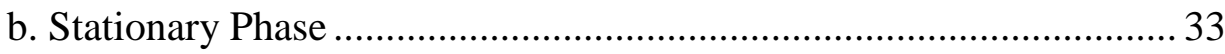

c. Voltage Effects and Joule Heating ................................................ 33

d. Ionic Strength ...................................................................................... 34

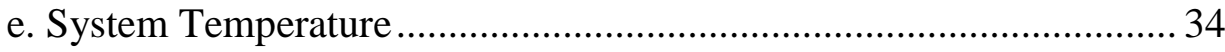

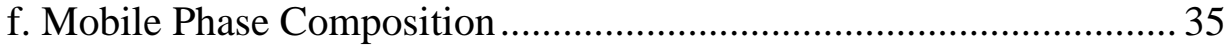

g. Capillary Considerations ……………………………...................... 35

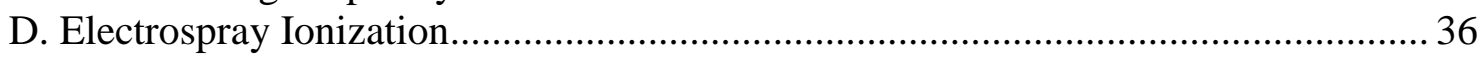

1. Production of Charged Droplets..................................................................... 36

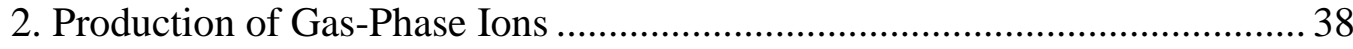

a. The Ion Evaporation Model............................................................... 38

b. The Charged Residue Model ............................................................ 38

3. Time-of-Flight Mass Spectrometry ……………………………………..... 39

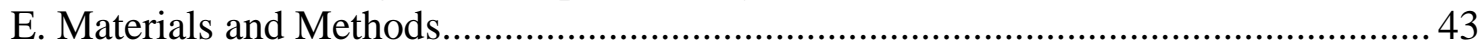

1. Reagents and Materials ............................................................................ 43

a. Monolith Preparation ................................................................... 43

b. Monolith Examination......................................................................... 44

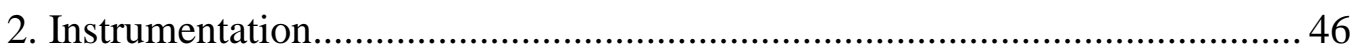

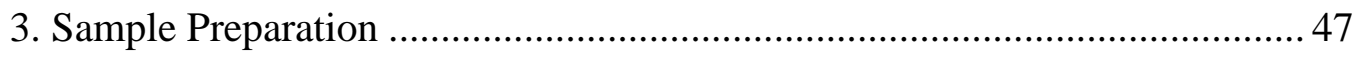

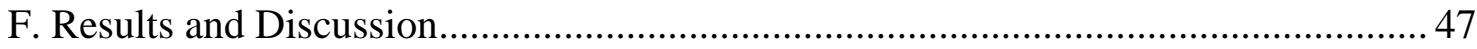

1. Method Development and CEC-UV Results ................................................ 48

2. Method Development and CEC-MS Results ................................................ 53

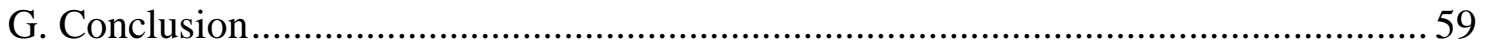


III. CHAPTER 3: Detection of the Improvised Explosives Ammonium Nitrate and Urea Nitrate using Nonaqueous Solvents with Electrospray Ionization

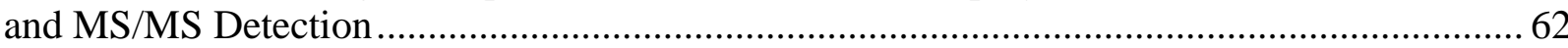

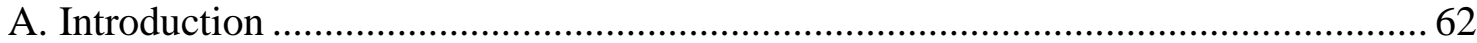

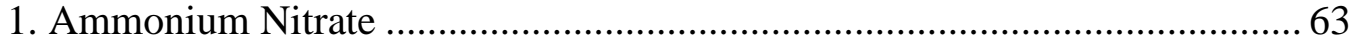

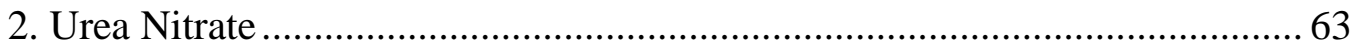

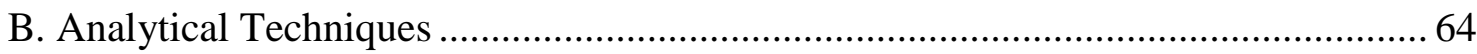

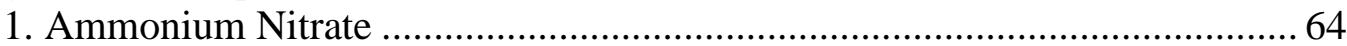

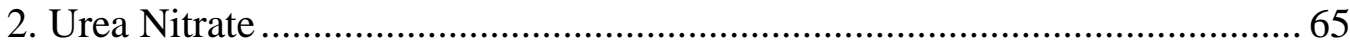

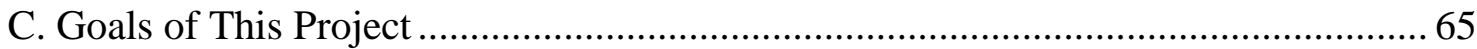

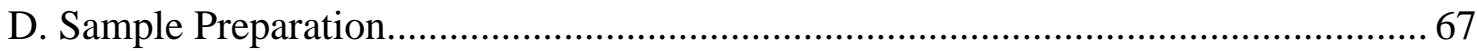

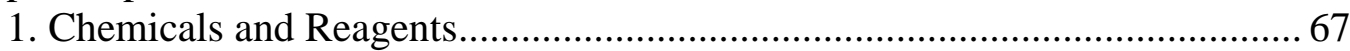

a. Choice of Dissolution Solvent for Urea Nitrate ............................. 67

b. Mechanism of $p$-DMAC Color Formation ..................................... 68

c. Choice of Dissolution Solvent for Ammonium Nitrate.................... 69

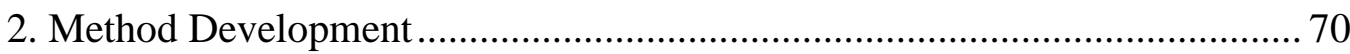

a. Instrumentation ....................................................................... 70

b. Ionization Parameters ..................................................................... 70

3. MS/MS Analysis and Detection Limits ....................................................... 71

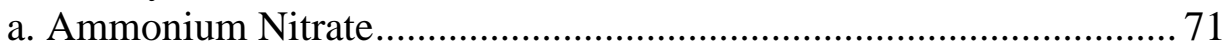

b. Urea Nitrate .................................................................................. 72

4. Differentiation of Urea Nitrate from Mixtures of Urea

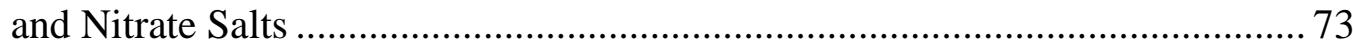

5. Detection of Ammonium Nitrate and Urea Nitrate in the Presence of Various Interfering Compounds ........................................................ 81

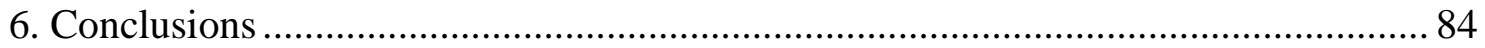

IV. Chapter 4: Development of a Paper Microfluidic Device for the Detection

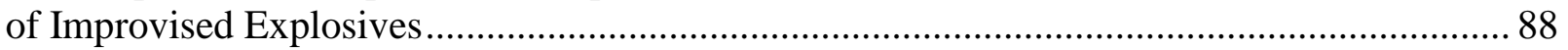

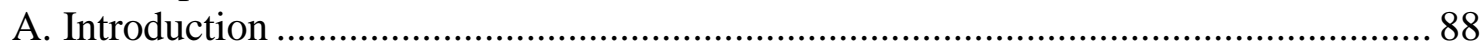

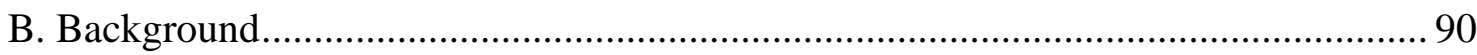

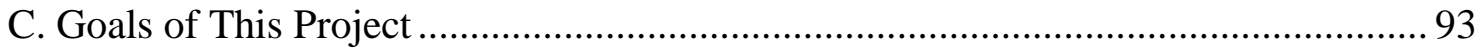

D. Development of the Paper Microfluidic Chip ....................................................... 94

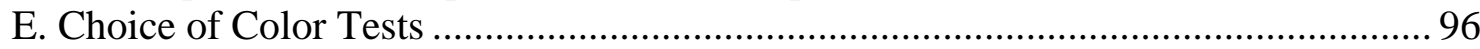

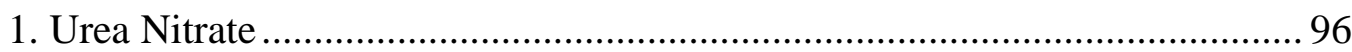

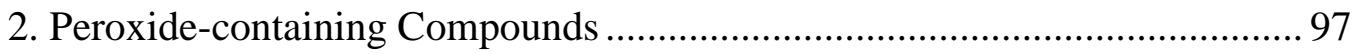

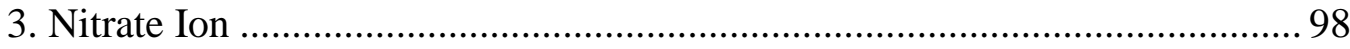

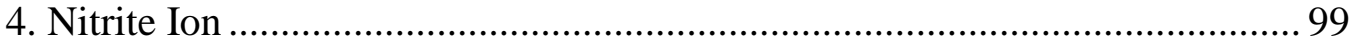

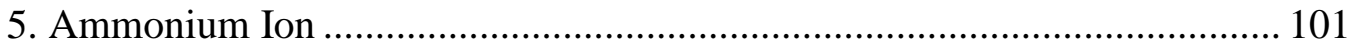

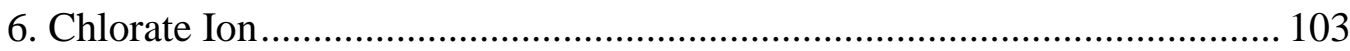

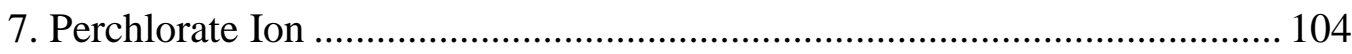

F. Performance Test of Inorganic Paper Microfluidic Device:

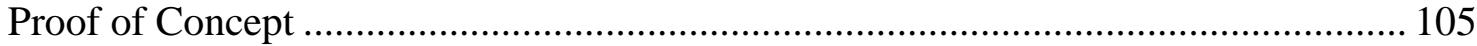

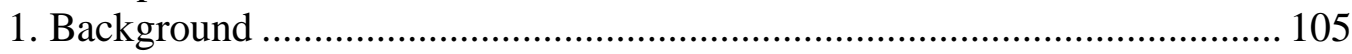

2. Sample Preparation ........................................................................... 106 


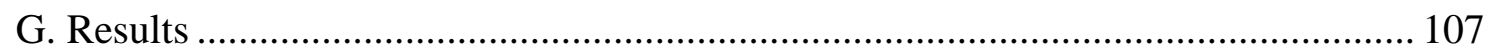

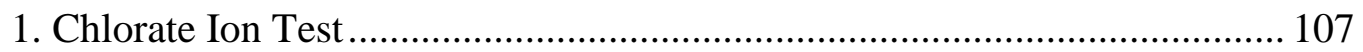

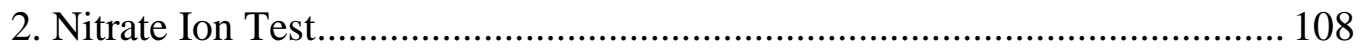

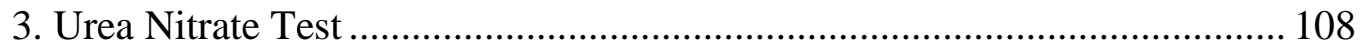

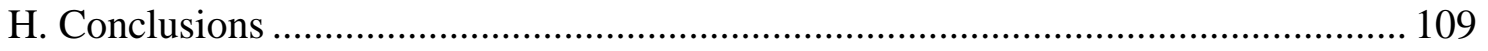

V. CHAPTER 5: Conclusions and Future Work .................................................................. 113

A. Separation and Identification of Organic Components of Smokeless

Gunpowder by Capillary Electrochromatography ………....................................... 113

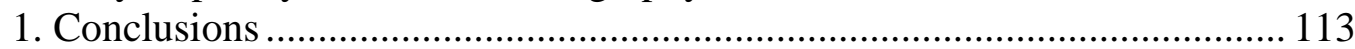

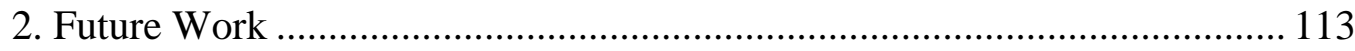

B. Detection of the Improvised Explosives Ammonium Nitrate and Urea Nitrate using Nonaqueous Solvents with Electrospray Ionization

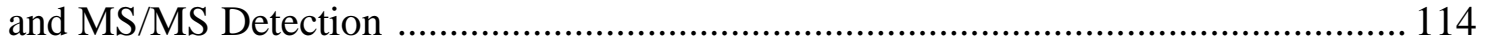

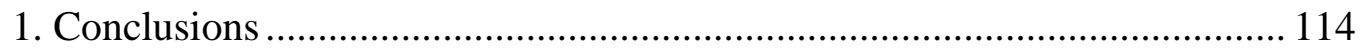

2. Future Work .................................................................................... 115

C. Development of a Paper Microfluidic Device for the Detection of Improvised Explosives ................................................................................. 115

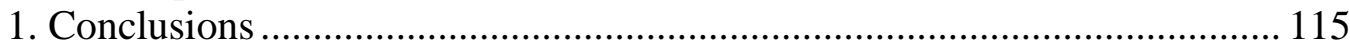

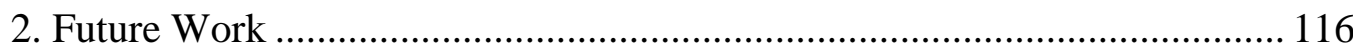

a. Pending Color Tests ................................................................... 116

b. Construction of the Final PMD Chip ………………….................... 117

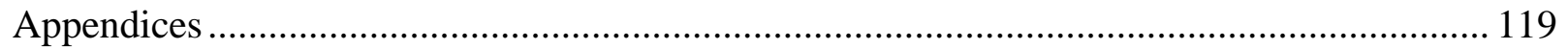

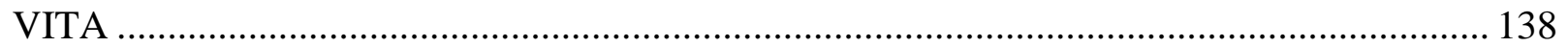




\section{LIST OF TABLES}

TABLE

PAGE

2-1 Figures of merit for CEC-UV. For linearity experiments $(n=9)$ with concentrations from 0 to $60 \mu \mathrm{g} / \mathrm{mL}$, the slope $(\mathrm{k})$ was calculated as area $=$ $\mathrm{k} \times$ concentration in $\mu \mathrm{g} / \mathrm{mL}$. DL is the detection limit, calculated from the signal obtained for the smallest concentration giving a peak and reported to a signal : noise ratio of 3 . For repeatability experiments $(n=5), 25 \mu \mathrm{g} / \mathrm{ml}$ of each compound was used. $\mathrm{N}$ is the number of theoretical plates. Conditions were as $73 / 27$ acetonitrile/phosphate buffer eluent, $30 \mathrm{kV}, 10 \mathrm{kV}$ for $8 \mathrm{~s}$ injection. 1. DMP, 2. MC, 3. DEP, 4. NG, 5. EC, 6. 3,4 DNT, 7. 2,4 DNT, 8. 2,6 DNT, 9. 4-NsDPA, 10. N-NsDPA, 11. DBP, 12. 4-NDPA, 13. DPA, 14. 2-NDPA.

2-2 Main adducts for smokeless powder additives standards detected in the negative and positive ion mode. The error on $\mathrm{m} / \mathrm{z}$ was calculated as the observed $\mathrm{m} / \mathrm{z}$ subtracted from the theoretical mass of the adduct and then divided by the theoretical mass.

2-3 Figures of merit for CEC-MS. For linearity experiments $(n=9)$, concentrations from 0 to $60 \mu \mathrm{g} / \mathrm{mL}$, the slope $(\mathrm{k})$ was calculated as area $=\mathrm{k} \times$ concentration in $\mu \mathrm{g} / \mathrm{mL}$. DL is the detection limit, calculated from the signal obtained for the smallest concentration giving a peak and reported to a signal:noise ratio of 3 . For repeatability experiments $(\mathrm{n}=4), 24 \mu \mathrm{g} / \mathrm{ml}$ of each compound was used. $\mathrm{N}$ is the number of theoretical plates. Conditions were as 73/27 acetonitrile/ phosphate buffer eluent, $30 \mathrm{kV}, 10 \mathrm{kV}$ for 8 s injection. 1. DMP, 2. MC, 3. DEP, 4. NG, 5. EC, 6. 3,4 DNT, 7. 2,4 DNT, 8. 2,6 DNT, 9/10. 4/N-NsDPA, 11. DBP, 12. 4-NDPA, 13. DPA, 14. 2-NDPA.

3-1 Response of UN on addition to different solvents. A positive result indicates the development of a red color with $\mathrm{p}-\mathrm{DMAC}$ reagent.........................69

3-2 Response of AN to dissolution in different solvents........................69

3-3 CID-MS peak data and product ion attribution........................... 79

3-4 Analysis of AN with interfering compounds: $1 \mathrm{~mL} 5 \mathrm{mM}$ AN standard, $5 \mu \mathrm{L}$ interferent. Dashes indicate complete suppression of the ion

3-5 Analysis of UN with interfering compounds: $1 \mathrm{~mL} 5 \mathrm{mM} \mathrm{UN}$ standard, interferent concentration as noted. Dashes indicate complete suppression of the

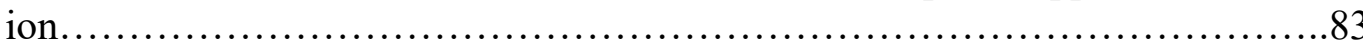

4-1 Results of proof of concept tests from extracted soil samples..................106 


\section{LIST OF FIGURES}

FIGURE

PAGE

2-1 Smokeless powder additives analyzed in this study: 1) dimethylphthalate (DMP), 2) methyl centralite (MC), 3) diethylphthalate (DEP), 4) nitroglycerin (NG), 5) ethyl centralite (EC), 6) 3,4-dinitrotoluene (3,4 DNT), 7) 2,4-dinitrotoluene (2,4 DNT), 8) 2,6-dinitrotoluene (2,6 DNT), 9) 4nitrosodiphenylamine (4-NsDPA), 10) N-nitrosodiphenylamine (N-NsDPA), 11) dibutylphthalate (DBP), 12) 4-nitrodiphenylamine (4-NDPA), 13) diphenylamine (DPA), 14) 2-nitrodiphenylamine (2-NDPA).

2-2 Simple schematic of a CEC instrument................................... 30

2-3 Graphic of the electrical double layer and distribution of charge [Barz, 26]........31

2-4 Flow profile comparison of laminar and electroosmotic flow...................32

2-5 Closeup of CEC-MS interface........................................... 37

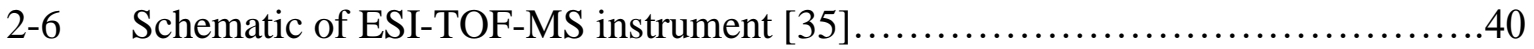

2-7 The reflectron corrects the kinetic energy dispersion of ions as they leave the

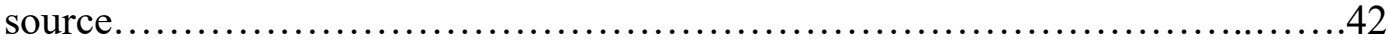

2-8 Scanning electron microscopy images of a.) successful monolith showing uniform polymerization; b.) closeup image of successful monolith; c.) unsuccessful monolith showing nonuniform polymerization; d.) closeup image of unsuccessful monolith

2-9 Electrochromatogram of a 14-compound mixture at $70 \mu \mathrm{g} / \mathrm{ml}$ each using a $73 / 27 \%$ acetonitrile/phosphate buffer eluent, $30 \mathrm{kV}, 10 \mathrm{kV}$ for 8 s injection. 1. DMP, 2. MC, 3. DEP, 4. NG, 5. EC, 6. 3,4 DNT, 7. 2,4 DNT, 8. 2,6 DNT, 9. 4-NsDPA, 10. N-NsDPA, 11. DBP, 12. 4-NDPA, 13. DPA, 14. 2-NDPA

2-10 Samples of (a) Winchester 296, (b) Hodgdon H380, (c) HI-SKOR 700-X, (d) IMR 4895, and (e) Red Dot Alliant Powder. Eluent was 79/21\% acetonitrile-phosphate buffer, $30 \mathrm{kV}$ running voltage, $10 \mathrm{kV}-8 \mathrm{~s}$ injection. For better clarity, electrochromatograms have been cropped and high intensity peaks were cut off. * indicates peaks too low to permit identity to be confirmed via UV spectrum. (1) DMP, (2) MC, (3) DEP, (4) NG, (5) EC, (6) 3,4-DNT, (7) 2,4-DNT, (8) 2,6-DNT, (9) 4-NsDPA, (10) N-NsDPA, (11)

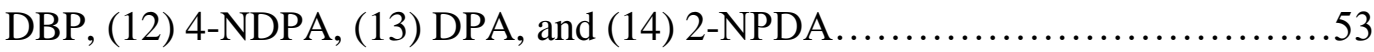


2-11 Chromatograms extracted at specific $\mathrm{m} / \mathrm{z}$ of a mixture of 14 standards at about $30 \mu \mathrm{g} / \mathrm{ml}$ each using a 73/27\% acetonitrile/phosphate buffer eluent, $30 \mathrm{kV}, 10 \mathrm{kV}$ for $8 \mathrm{~s}$ injection, source temperature $=150{ }^{\circ} \mathrm{C}$, drying gas $=5$ $\mathrm{ml} / \mathrm{min}$, nebulizer pressure $=10 \mathrm{psig}$. In the positive ion mode (ESI+), capillary $=4000 \mathrm{~V}$, fragmentor $=140 \mathrm{~V}$, skimmer $=50 \mathrm{~V}$. In the negative ion mode $(\mathrm{ESI}-)$, capillary $=5000 \mathrm{~V}$, fragmentor $=125 \mathrm{~V}$, skimmer $=80 \mathrm{~V}$. (1) DMP, (2) MC, (3) DEP, (4) NG, (5) EC, (6) 3,4-DNT, (7) 2,4-DNT, (8) 2,6-DNT, (9) 4-NsDPA, (10) N-NsDPA, (11) DBP, (12) 4-NDPA, (13) DPA, and (14) 2-NDPA.

3-1 Resonance structures of the colored complex formed with urea nitrate and $p$ DMAC. .68

3-2 Positive ESI mass spectrum of $10 \mathrm{mM}$ ammonium nitrate (AN) standard........72

3-3 Negative ESI mass spectrum of $10 \mathrm{mM}$ urea nitrate (UN) standard ...................73

3-4 Comparison of negative ESI mass spectrum of $10 \mathrm{mM} \mathrm{UN}$ (top) with negative ESI mass spectra of $10 \mathrm{mM}$ urea with varying amounts of AN. Note the presence of the $\mathrm{m} / \mathrm{z} 182$ peak in the mixtures; this peak is not present in a true UN sample. Also note that in true UN the ratio of the 248:185 peaks is greater than

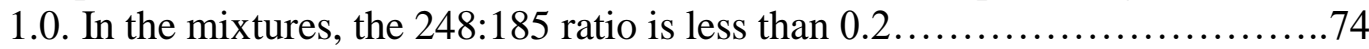

3-5 Closeup of negative ESI spectrum of urea and AN mixtures with urea held constant at $10 \mathrm{mM}$...

3-6 Comparison of negative ESI mass spectrum of $10 \mathrm{mM} \mathrm{UN}$ (top) with negative ESI mass spectra of $10 \mathrm{mM}$ AN using varying amounts of urea.................76

3-7 Collision-induced dissociation mass spectrometry (CID-MS/MS) was performed to determine the identity of the product ions of the $\mathrm{m} / \mathrm{z} 182$ peak. Identity and masses are labeled..................................................76

3-8 Collision-induced dissociation mass spectrometry (CID-MS/MS) was performed to determine the identity of the product ions of the $\mathrm{m} / \mathrm{z} 185$ peak. Identity and masses are labeled..................................................... 77

3-9 Collision-induced dissociation mass spectrometry (CID-MS/MS) was performed to determine the identity of the product ions of the $\mathrm{m} / \mathrm{z} 242$ peak. Identity and masses are labeled

3-10 Collision-induced dissociation mass spectrometry (CID-MS/MS) was performed to determine the identity of the product ions of the $\mathrm{m} / \mathrm{z} 248$ peak. Identity and masses are labeled 
3-11 Top: $10 \mathrm{mM}$ UN standard. Bottom: $10 \mathrm{mM}$ urea $+5 \mathrm{mM}$ AN. Without a proton source, no UN signal is generated.................................80

3-12 Top: $10 \mathrm{mM}$ urea $+10 \mathrm{mM} \mathrm{KNO}_{3}$. Bottom: $10 \mathrm{mM}$ urea $+10 \mathrm{mM} \mathrm{NH} \mathrm{NO}_{3}$. Without a proton source, no UN signal is generated.......................... 80

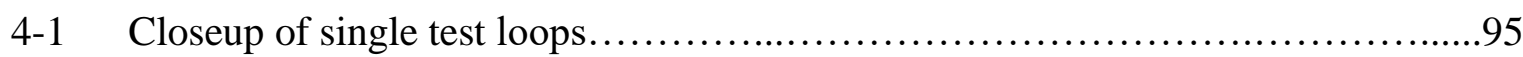

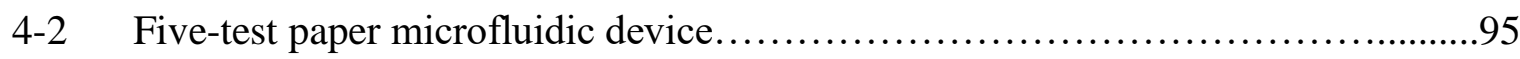

4-3 Positive result for $\mathrm{p}$-DMAC reagent with urea nitrate dissolved in methanol and run immediately after being put into solution...........................96

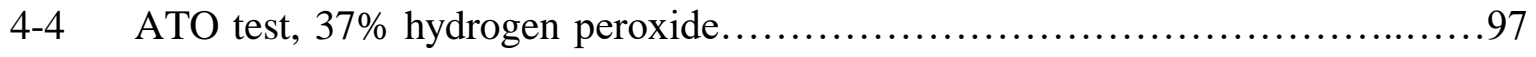

4-5 ATO test, TATP (blank left, TATP right) .................................97

4-6 Color formation mechanism of ATO with hydrogen peroxide....................97

4-7 Nitrate test result. Modified from Niki et al. [26]............................98

4-8 Color formation mechanism for the nitrate ion test...........................99

4-9 Nitrite test result using 1-naphthylamine............................... 100

4-10 Color formation mechanism for the nitrite ion test.......................... 100

4-11 Addition of BCDMA, method [25] ..................................... 102

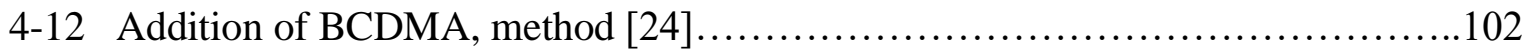

4-13 Reaction mechanism for the formation of the reddish-brown color associated with Nessler's test for the ammonium ion.......................103

4-14 Positive test result for chlorate ion using aniline sulfate.......................104

4-15 Color formation mechanism for the chlorate ion test.........................104

4-16 Positive test result for perchlorate ion using methylene blue....................104

4-17 Color formation mechanism for the perchlorate ion test...................... 105

4-18 PMD developed for initial performance test............................ 105

4-19 Representative positive chlorate test.................................108

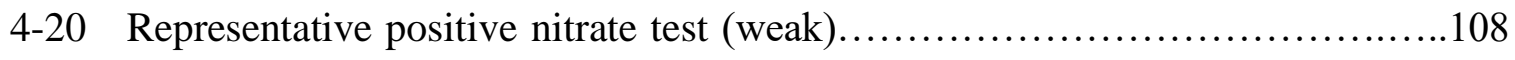

4-21 Positive urea nitrate test............................................ 109 


\section{LIST OF ABBREVIATIONS AND SYMBOLS}

\begin{tabular}{|c|c|}
\hline $\mathrm{ACN}$ & acetonitrile \\
\hline AMPS & 2-acrylamido-2-methylpropane sulfonic acid \\
\hline AN & ammonium nitrate \\
\hline ATO & ammonium titanyl oxalate \\
\hline $\mathrm{E}$ & applied electrical field \\
\hline APCI & atmospheric pressure chemical ionization \\
\hline AIBN & azobisisobutyronitrile \\
\hline BCDMA & benzylcetyldimethylammonium chloride \\
\hline BDDA & 1,4-butanediol diacrylate \\
\hline CEC & capillary electrochromatography \\
\hline $\mathrm{CE}$ & capillary electrophoresis \\
\hline CI & chemical ionization \\
\hline CID-MS & collision-induced dissociation - mass spectrometry \\
\hline DBP & dibutyl phthalate \\
\hline DCI & dichloroisocyanuric acid \\
\hline$\varepsilon_{\mathrm{r}}$ & dielectric constant \\
\hline DEP & diethyl phthalate \\
\hline DMF & dimethylformamide \\
\hline DMP & dimethyl phthalate \\
\hline 2,4-DNT & 2,4-dinitrotoluene \\
\hline 3,4-DNT & 3,4-dinitrotoluene \\
\hline
\end{tabular}




$\begin{array}{ll}\text { 2,6-DNT } & \text { 2,6-dinitrotoluene } \\ \text { DAD } & \text { diode array detector } \\ \text { DPA } & \text { diphenylamine } \\ \text { EDL } & \text { electrical double layer } \\ \text { EI } & \text { electron ionization } \\ \text { EOF } & \text { electroosmotic flow } \\ \mu_{\text {eo }} & \text { electroosmotic mobility } \\ u_{\text {eo }} & \text { electroosmotic flow velocity } \\ \text { ESI } & \text { electrospray ionization } \\ \text { EC } & \text { ethyl centralite } \\ \text { FBI } & \text { Federal Bureau of Investigation } \\ \text { FID } & \text { flame ionization detector } \\ \text { FTIR } & \text { Fourier transform infrared } \\ \text { GC/MS } & \text { gas chromatography/mass spectrometry } \\ \text { HMTD } & \text { hexamethylene triperoxide diamine } \\ \text { HA } & \text { hexyl acrylate } \\ \text { HPLC } & \text { high-performance liquid chromatography } \\ \text { HME } & \text { homemade explosive } \\ \text { IED } & \text { improvised explosive device } \\ \text { ICP-MS } & \text { inductively coupled plasma mass spectrometry } \\ \text { kV } & \text { kilovolt } \\ \text { kinetic energy }\end{array}$




\begin{tabular}{|c|c|}
\hline LC/UV & liquid chromatography/ultraviolet detection \\
\hline MS & mass spectrometry \\
\hline $\mathrm{MC}$ & methyl centralite \\
\hline MEKC & micellar electrokinetic chromatography \\
\hline$\mu \mathrm{L}$ & microliter \\
\hline $\mathrm{mM}$ & millimolar \\
\hline $\mathrm{pH}$ & negative logarithm of the hydrogen ion concentration \\
\hline ESI- & negative mode electrospray ionization \\
\hline 2-NDPA & 2-nitrodiphenylamine \\
\hline 4-NDPA & 4-nitrodiphenylamine \\
\hline NG & nitroglycerin \\
\hline 4-NsDPA & 4-nitrosodiphenylamine \\
\hline N-NsDPA & N-nitrosodiphenylamine \\
\hline$u_{\mathrm{obs}}$ & observed net migration velocity \\
\hline PMD & paper microfluidic device \\
\hline$p$-DMAC & para-(dimethylamino)cinnamaldehyde \\
\hline PETN & pentaerythritol tetranitrate \\
\hline$\varepsilon_{0}$ & permittivity of vacuum \\
\hline PDMS & polydimethylsiloxane \\
\hline ESI+ & positive mode electrospray ionization \\
\hline$u_{\text {press }}$ & pressurized flow velocity \\
\hline $\mathrm{h}$ & reduced plate height \\
\hline RDX & Research Department explosive \\
\hline
\end{tabular}




\begin{tabular}{|c|c|}
\hline $\mathrm{R}_{\mathrm{s}}$ & resolution \\
\hline $\mathrm{k}$ & retention, capacity factor \\
\hline SEM/EDS & $\begin{array}{l}\text { scanning electron microscopy/energy dispersive X-ray } \\
\text { spectroscopy }\end{array}$ \\
\hline $\mathrm{NaOH}$ & sodium hydroxide \\
\hline $\mathrm{H}_{2} \mathrm{SO}_{4}$ & sulfuric acid \\
\hline TOF-MS & time of flight mass spectrometry \\
\hline TATP & triacetone triperoxide \\
\hline TFA & trifluoroacetic acid \\
\hline TNT & trinitrotoluene \\
\hline UV & ultraviolet detection \\
\hline UN & urea nitrate \\
\hline VBIED & vehicle-borne improvised explosive device \\
\hline$\eta$ & viscosity \\
\hline $\mathrm{XRF}$ & $\mathrm{X}$-ray fluorescence \\
\hline XRD & X-ray powder diffraction \\
\hline$\zeta$ & zeta potential \\
\hline
\end{tabular}




\section{CHAPTER 1: A Short History of Improvised Explosives and Devices}

\section{A. Introduction}

An improvised explosive device (IED) is a "homemade" explosive device built with materials at hand and designed to cause death or injury [1]. It may also be defined as "a criminally fabricated device incorporating destructive, lethal, noxious, pyrotechnic or incendiary chemicals, and designed to destroy, disfigure, distract, or harass. It may incorporate military stores but normally is designed from commercial or homemade components [2].”

Improvised explosive devices have appeared in warfare for thousands of years, although

the term has only recently been established. From the Greek wars in the $7^{\text {th }}$ century to the battlefields of Afghanistan and other parts of south-central Asia, many forms of IEDs have been used in war and as political tools. They may be of simple construction or mechanically complex; the explosive charge may be anything from stable military explosives to improvised materials that include dangerously unstable primary explosives.

Explosives have a similarly long history of use. Among the first use of these materials was the compound called "Greek fire," developed by the Eastern Roman (Byzantine) Empire for use in naval wars. The exact composition of this incendiary weapon has been lost to antiquity. However, it is known to have been a burning liquid that coated and burned whatever surface on which it was sprayed, and it could not be extinguished with water. Its discovery is credited to Kallinikos, a Syrian engineer. He prepared "marine fire" to burn the boats of the Saracens, who had come to lay siege to Constantinople [3]. There are various proposed recipes for Greek fire. Some include pitch or other petroleum 
products collected from natural hydrocarbon seeps and mixed with sulfur and niter (potassium nitrate) [4]. Others propose the addition of calcium oxide [5], which generates substantial heat on contact with water and with the addition of a tinder such as sulfur may sustain combustion. The important issue in the development of Greek fire was the addition of an oxidizer which permitted the material to burn or deflagrate in the absence of air. In fact this was one of the first descriptions of a mixture that contained a combination of oxidizer and fuel was a critical development in the history of explosives. The origin of black powder is another undiscovered secret. There is dispute as to who was the first to describe the components of black powder in Europe, but Roger Bacon is generally given credit. He was an English monk who in the late 1260s translated an Arabic document that was derived from an earlier Chinese text that laid out a basic formula for black powder. After years of experimentation, the optimum formula was determined to be fifteen parts potassium nitrate, three parts charcoal, and two parts sulfur. Initially black powder was used to break rock in mines but soon it changed the face of warfare. Black powder was used to charge petards and primitive cannons that hurled stones and arrows at enemies and fortifications. Black powder was also used in early improvised explosive devices (IEDs) in efforts to break sieges and to frighten enemies.

As time progressed, new technological developments were incorporated into the construction of IEDs. While black powder continued to make up the charge, initiation mechanisms became more sophisticated. Flintlock and wheelock based triggers were incorporated into firearms to provide the initial spark to the powder charge. The other $16^{\text {th }}$ century invention that enhanced the development of IEDs was the clock. A clock- 
based initiator was used during the Siege of Antwerp in 1584, during the Eighty Years' War.

The Spanish Army had the city of Antwerp blockaded. The use of fire ships - vessels that sailed close to the enemy and ignited - was common, but this new technology when combined with the use of gunpowder would take marine warfare to a new level. A ship called the Hoop ("Hope") was loaded with 7,000 pounds of black powder and filled with shrapnel including cannonballs, iron hooks, and harpoons. The initiator was a modified clock with a time delay. The prepared ship was sent against a blockaded bridge. When the clock reached the appointed time, a firearm lock was activated. The resulting explosion killed between 800 and 1000 Spanish troops [6]. With no way to detect or guard against another such incident, the effectiveness of the Spanish Armada was seriously disrupted for many years by the memory of the Hoop and the fear that another ship like it would be used against them.

Black powder was used as the main charge in weaponry and improvised devices for hundreds of years. One of the first recorded instances of the use of a vehicle-borne improvised explosive device (VBIED) was in the assassination attempt on Napoleon Bonaparte on Christmas Eve, 1800. The infernal machine was constructed by loading a cart with barrels of gunpowder and leaving the loaded cart along the route Napoleon and Josephine were to take to the opera. The bomb was to be ignited with a long fuse when the signal was given and explode as the royal coach and bodyguards passed by. However, the couple was running late. The speeding coach passed the VBIED and turned 
the corner before it exploded. Many bystanders and two of the guards were killed, but the royal couple was unhurt [7].

Black powder was also used during the US Civil War by the Confederacy in a device called the coal torpedo. It was an IED produced by taking a hollow shell of random shape made of iron and filled with gunpowder. It was next sealed with wax and covered with coal dust, so that when hidden in a coal pile it would be shoveled into the furnace of a steam-powered Union ship [8]. While the coal torpedo was not powerful enough to destroy a ship outright, the resulting explosion could compromise the ship's boiler, causing a pressure failure that would destroy the vessel. With no way to detect the dangerous lookalikes, Union soldiers were given orders to shoot on sight anyone lurking around the coal stockpiles.

In 1847 Ascanio Sobrero, an Italian chemist at the University of Turin, treated glycerin with concentrated nitric acid and found it reacted violently. After modifying the procedure to include the use of a mixture of sulfuric and nitric acids, and the use of an ice bath to cool the reaction, he produced nitroglycerin [9]. He found it too unstable and hard to work with, and kept his discovery secret for a year before publicizing it. His work, however, became the basis of Alfred Nobel's development of dynamite. The syntheses of nitroglycerin and nitrocellulose (guncotton) also ultimately yielded modern smokeless gunpowder. These advancements added a new material for use in IEDs.

Bombings continued in the $19^{\text {th }}$ and early $20^{\text {th }}$ century in both Europe and the US as tools of political movements. Anarchists, separatists, and groups that wanted their causes publicized resorted to IEDs to make their point. To combat the waves of violence, new 
technology was developed. In 1895 Wilhelm Röentgen began his first detailed study of X-rays. By the late 1890s, French police made radiographs of booby-trapped packages to assist them in disarming the devices [10]. Between the mid 1870's and the post-World War II era, dynamite was the most common charge used in explosive devices, and many incidents took place in the United States and Europe $[11,12,13]$. The work performed in combatting these violent acts resulted in the development of dedicated bomb squads and safety measures such as body armor.

Improvised explosive devices (IEDs) first became a topic of concern for the American public with the beginning of the war in Afghanistan after September 11, 2001, and after the 2003 invasion of Iraq. Between the two campaigns, approximately 3,300 coalition soldiers were killed by IEDs [14]. Initially described as "roadside bombs," the term "improvised explosive device" did not officially become part of American lexicon until 2007 [15]. In the early days of the war, devices were constructed from military explosives stolen from coalition forces or left behind by Soviet troops fighting in Afghanistan in the 1980's. As time progressed, security was tightened, and the supply of Russian ordnance was depleted. The main charge in improvised devices increasingly became ammonium nitrate (AN) with a hydrocarbon or metal fuel. After the use of AN fertilizer was banned in Afghanistan in 2010 [16], potassium chlorate, and to a lesser extent urea nitrate (UN), became more common as the oxidizers used in IEDs. Additionally, insurgents made their own improvised blasting caps and synthesized the primary explosives to fill them.

As groups such as al-Qaeda and Daesh (also called ISIL or ISIS) move out of the Arabian Peninsula and into other parts of the world, such as Africa and Southeast Asia, concerns 
about IEDs deployed against other military targets and civilian populations have become a greater concern globally. In the United States, the threat of domestic terrorism and the easy online access to explosive synthesis information and device construction have become concerns for law enforcement, leading to expanded information sharing and cooperation between local and national agencies. In addition to new investigative techniques, the forensic analysis of explosives has become part of the national strategy for combating both foreign and domestic terror. The discipline of battlefield forensics has emerged and now the techniques and goals of forensic analysis are found on battlefields all over the world.

For battlefield examinations, explosives may be submitted as either bulk samples or postblast residue. In the case of post-blast residue the device may have functioned as intended and the sample was collected as part of the investigation, or the device may have been detonated for safety reasons and pieces of the device with residue or swabbings from stationary objects may be submitted.

\section{B. Overview of Explosives}

An explosive is "a material, either a pure single substance or a mixture of substances, which is capable of producing an explosion by its own energy [17]. They may be grouped by many different properties. One of the most basic is the differentiation between high and low explosives. 


\section{High Explosives}

A high explosive detonates, producing a shock front that moves through the unreacted explosive at a velocity greater than the speed of sound, producing very high pressures and temperatures. Behind the shock front is a slower-moving chemical reaction zone where the original material is rapidly converted into reaction products [18] and a great amount of energy. High explosives may be further divided into primary and secondary explosives. Primary explosives are compounds that are sensitive to physical shock, friction, heat, flame, and electrical discharge [19]. Examples of primary explosives include lead azide and mercury fulminate. Secondary explosives are less sensitive and require an initiator that contains a primary explosive in order to be detonated. Secondary explosives include TNT, RDX, and PETN.

\section{Low Explosives}

A low explosive undergoes a particle-to-particle burning transition called a deflagration. The reaction proceeds too slowly to produce the shock wave that is the mark of a detonation [20]. A deflagration event moves through the material at a velocity less than the speed of sound in the unreacted material. The burning produces high temperatures and large volumes of gaseous reaction products. Low explosives include small arms propellants such as smokeless powder, black powder and black powder substitutes, and flash powders. All of these must be confined in a container, such as a metal pipe, that will allow pressure to build up until the container ruptures. 
Tertiary explosives, also called blasting agents, are primarily AN-based. The AN functions as the oxidizer and various fuels, such as diesel fuel, sugar, or finely divided metals are added to react with the oxidizer.

\section{Improvised Explosives}

The final category, improvised explosives, covers a wide variety of compounds both organic and inorganic with many different physical and chemical properties. The most common characteristic that improvised explosives share is that they are made from common household or industrial chemicals that cannot be monitored or controlled without imposing undue hardships on consumers or industry. While some starting materials are now hard to get for legal or environmental reasons, the pool of available options is still large. The FBI has produced an IE threat card that outlines many common fuel-oxidizer combinations and their precursors [21].

\section{a. Smokeless Powders}

Smokeless powders are propellants used in pistol and rifle ammunition. They are called "smokeless" because, in comparison to black powder, they generate very little smoke and minimal residue, so weapon barrels are not fouled the way they would be with black powder. The designations of the three types of smokeless powder are based on the energetic materials found in each one. Single-base powder contains only nitrocellulose as the energetic material, while double-base powder contains nitrocellulose and nitroglycerin. Both are used in cartridges for pistols and shotguns. The third type, triplebase powder, contains nitrocellulose, nitroglycerin, and nitroguanidine. Triple-base 
powder is used for military weaponry, such as naval guns and is not available commercially. Smokeless powder forms are made by mixing the different components with an organic solvent into a doughy mass and extruding the powder through a die to produce its final shape. In addition to the energetic components, modern smokeless powder formulations include stabilizers, plasticizers, flash suppressants, deterrents, opacifiers, and dyes. These components in the powder are known as the "additive package."

Stabilizers are added to the powder to scavenge the nitric and nitrous acids produced by the decomposition of nitrocellulose and nitroglycerin. If left to accumulate, nitric and nitrous acids can catalyze a decomposition reaction in the powder. Common stabilizers used to combat this problem are diphenylaminie, methyl and ethyl centralites. When present in a smokeless powder, diphenylamine reacts to produce a variety of nitro- and nitrosodiphenylamine compounds. The concentration and variety of these nitrated stabilizers in a powder may be used as a measure of its age or lot number [22, 23], so determination of the identity and concentration of these products is an important component of a forensic analysis [24].

Plasticizers reduce the need for volatile solvents during mixing and extrusion of the powder. Phthalates are commonly-used plasticizers. Nitroglycerin, although it functions as a primary energetic material in smokeless powder, is also a plasticizer that decreases the need for volatile solvents in the manufacturing process. Flash suppressants are typically alkaline earth metals. They reduce muzzle flash when the weapon is fired. 
Deterrents and opacifiers are surface coatings that ensure a consistent burn rate and improve the efficiency of the powder. Deterrents include methyl or ethyl centralite and phthalates. Although phthalates such as dioctyl or dibutyl phthalate function as plasticizers, they also have a deterrent effect. The most common opacifier is carbon black, which also reduces static.

Dyes may be added to assist in the identification of some brands of powder. All of the additional components that are added to the nitrocellulose polymer are termed the additive package, and can vary between different brands and formulations.

Given the implications for law enforcement and national security organizations, much work has been devoted to the identification of smokeless powders by characterizing the additive package [25 - 29]. When used as the filler for a pipe bomb, a portion of unburned material may be thrown clear of the device and if found by investigators, the chemical composition of the unexploded powder can be used to assist in the determination of its origin.

\section{b. Ammonium Nitrate and Urea Nitrate}

Ammonium nitrate $(\mathrm{AN})$ and urea nitrate $(\mathrm{UN})$ are salts of nitric acid that are used in IEDs. AN, represented by the formula $\mathrm{NH}_{4} \mathrm{NO}_{3}$, is a common fertilizer that was first used as a blasting agent in 1867 . Over the years, AN has become the most widely used mining explosive and is generally mixed with $6 \%$ fuel oil prior to use [30]. Ammonium nitrate is also routinely used in improvised explosive devices. It has received much attention for its use in the Middle East and Southern Asia, including Afghanistan and Pakistan. In the 
United States, it was most notably used to destroy the Alfred P. Murrah Federal Building in 1995 [31].

Urea nitrate was mostly unknown to the general public until it was used in the first World Trade Center bombing in 1993. It is sometimes used as an alternative to AN in improvised devices. Both AN and UN are easily obtained or synthesized from unregulated materials and have been employed in bombing incidents around the world. AN (with PETN) was used in the Delhi High Court bombing in 2011 [32] and UN has been used by Palestinian separatists against the Israeli military [33]. The importation of certain types of fertilizer was banned by the Peruvian government in 1992 because UN was a favorite explosive of the Shining Path guerilla organization [34], and the government of Afghanistan has banned the use of AN because of its diversion from farming to production of improvised explosives [35]. UN is also a popular topic of discussion on "citizen scientist" [36, 37] websites because of its easily sourced ingredients and ease of manufacture.

$\mathrm{UN}$ can be represented by the formula $\mathrm{NH}_{2} \mathrm{CONH}_{2} \bullet \mathrm{HNO}_{3}$. UN is a stable tertiary explosive [38]; however, the finished compound is very acidic. The nitric acid moiety is only loosely bonded to the urea molecule and attacks metal, including containers that might be used for storage or delivery of the explosive. Because of its acidic nature UN is synthesized and used immediately, not stockpiled for later use. In aqueous solution, UN breaks down into urea and nitric acid. Because of this breakdown, any analytical technique that is designed to detect UN must be capable of discriminating between urea and urea nitrate. 
This metathetical reaction became a point of contention during the investigation of the first World Trade Center attack in 1993 when it was pointed out by an FBI chemist that the metathetic reaction of urea nitrate might produce a false positive during mass spectral analysis. Since sewage pipes had been ruptured in the explosion, it was possible that liquid waste containing urea and nitrates had contaminated the samples being analyzed. Other results from infrared spectra of crystalline material obtained at the bomb-making site (a storage locker) revealed the presence of urea nitate in addition to the presence of starting materials. However, the mass spectral data from the seat of the explosion was not sufficient to confirm the presence of this explosive, and the infrared technique was not sufficiently selective or sensitive to detect urea nitrate. Ultimately, laboratory reports were written to state that urea nitrate could not be conclusively identified in the samples taken from the seat of the explosion, and only the infrared data from the bomb making site was used to identify urea nitrate as the explosive [66]. A better method was needed for this analysis.

\section{c. Improvised Explosive and Pyrotechnic Compositions}

Commonly used materials for improvised explosive preparations may include various oxidizers, inorganic fertilizers, industrial chemicals, and consumer products. Fuels used in these compositions may include metallic particles or carbon-containing organic compounds, such as hydrocarbons. Because the fuels and oxidizers span a wide range of properties including solubility, polarity, and volatility, a number of different analytical techniques are required for full characterization of these compounds. Oxidizers such as peroxides, nitrates, and perchlorates require FTIR, GC/MS, or LC/MS analysis for 
identification. Metal fuels such as aluminum, magnesium, or zinc require $\mathrm{X}$-ray diffraction (XRD), X-ray fluorescence (XRF), or ICP-MS analysis for identification. Carbon-based fuels may be identified by GC/MS, polarized light microscopy, or infrared techniques. Fast preliminary identification of these components in the field can save time and allow first responders to embark on a course of action before full characterization of a sample is completed.

\section{Forensic Application of Explosives Analysis}

Because typically there is a need for rapid analysis of explosives in both bulk and trace forms at crime scenes, sensitive and specific methods of detection are needed to determine the presence and identity of explosives in forensic samples. The detection and identification of commercial and improvised explosives requires the development of fast and accurate presumptive tests that can be used at the scene. These new analytical methods must also be capable of differentiating explosive mixtures from closely-related non-explosive mixtures.

In the field or at the scene of a bombing, first-line tools such as colorimetric tests and portable instrumentation are used to make preliminary determinations that help investigators determine what explosives were used. Color tests work by generating an immediate and obvious color change when the sample is combined with a test reagent. They are easy to use, inexpensive, portable and do not take up much space. They give clear and unambiguous results that are easy to read and interpret. 
Portable instrumentation such as Raman or FTIR spectrometers share many of the advantages of color tests. While they are more expensive, they are made for ease of use and do not require long training periods to generate good results. Results are available within minutes, which allows first responders to take action without waiting for the sample to be sent to a remotely located laboratory and analyzed.

While on-scene tools generate fast results, they do have limitations. Color tests, being presumptive, are subject to false positive results. Portable instrumentation may not be sensitive enough to detect minute quantities of a substance, or the instrument may not use the appropriate physical principles to detect the compound of interest. In these cases analysis in the laboratory is needed.

Laboratory instrumentation is more expensive than field-portable equipment, sometimes by several orders of magnitude, and cannot be brought to the scene because of its size or because it requires such support as conditioned power, compressed gases, or vacuum pumps to operate. In the laboratory, though, a more thorough characterization of samples can be done. The lower detection limits associated with laboratory-based equipment give additional information to be acted upon and confirmatory tests performed in the laboratory conclusively identify the components of a sample.

Considerable work has been devoted to the analysis of the additive package of smokeless powders $[24-29,39,40]$. A comprehensive review of these procedures was conducted by Meng and Caddy [41]. A wide variety of techniques and technologies have been applied ranging from gas chromatography/mass spectrometry to spectroscopy. Determination of age or lot number may also be possible in certain situations. The 
presence of smokeless powder on skin or clothing has also been used indicate that a person has been near a weapon when it was fired.

Gas chromatographic methods are traditionally used to separate the different components of smokeless powder samples; however, control of injector conditions is important to avoid thermal decomposition [42] of nitrated organic compounds such as nitroglycerin. To overcome this limitation, both high performance liquid chromatography (HPLC) and capillary electrophoresis (CE) methods have been developed [29, 43 - 45]. Micellar electrokinetic chromatography techniques (MEKC) $[46,47]$ overcame the limitations of traditional CE by allowing neutral molecules to be separated and offered improved resolution of nitrotoluene isomers. However, the use of nonvolatile surfactants in the buffer makes it impractical to couple MEKC to a mass spectrometer.

AN may be identified by electrospray ionization - mass spectrometry (ESI-MS) in positive ionization mode [48]. Ammonium and nitrate ions may be identified by ion chromatography [49] or color test reagents such as the diphenylamine test for nitrate ion or the Nessler reagent for ammonium ion, but no color test for the AN pair exists. UN may be presumptively identified by using para-dimethylaminocinnamaldehyde ( $p$ DMAC) [50]. UN may also be identified by XRD [51], infrared spectroscopy [52], voltammetry [53], in positive mode ESI-MS when complexed with crown ethers [54, 58], and by LC/UV-fluorescence using xanthydrol as a fluorophore [55]. Almog expanded on the use of xanthydrol for the detection of UN by a further reaction with alcohol to form a compound amenable to GC/MS analysis [56]. Residual amounts of AN [48] and UN [57] may be quickly and selectively determined by ESI-MS. Photodissociation followed by 
laser-induced fluorescence has also been used for both compounds [59]. Both UN and $\mathrm{AN}$ are loosely bound ionic pairs that undergo a metathetical reaction and combine with other salts when dissolved in polar protic solvents such as water.

In the field, the use of analytical instrumentation has its drawbacks: some instrumentation is not portable due to power, compressed gas, or vibration isolation requirements. Battery power may be drained before the work is completed. Colorimetric and immunoassay based tests have been developed, but are currently not multiplexed, and may require multiple tests and reagents, extending analysis time and increasing the amount of sample needed for testing $[60,61]$. Paper microfluidic devices have attracted attention as fast, inexpensive field testing devices in healthcare applications [62]. Reagents are applied directly to the paper within patterned channels and the sample, in an appropriate solvent, is introduced onto the paper. The wicking action removes the need for pumps. Because no reagents are used, there are fewer disposal issues for the used tests; if disposal is needed, they can be burned.

Paper microfluidic devices (PMDs) have been used to detect organic explosives [63], peroxide and nitrobenzenes [64], and trinitroaromatics [65], but no attempt has been made to detect fertilizer-based explosives or pyrotechnic compounds prior to this work.

\section{Goals of This Research}

There were several goals of this research: the first was to develop and validate electrospray ionization mass spectrometry (ESI-MS) methods for the detection and identification of organic smokeless powder additives. Separation was accomplished by 
capillary electrochromatography (CEC) using a novel hexyl acrylate based porous monolith as a stationary phase, and detection was performed by UV and electrospray ionization-time of flight mass spectrometry (ESI-TOF-MS). The CEC-UV method is an efficient one for detecting all components in the additive package of smokeless powders, and ESI-TOF-MS provides better sensitivity and selectivity. After detecting the compounds by positive- and/or negative-ion electrospray ionization mass spectrometry, CEC allowed confirmation of the compounds present.

The second goal was to develop and validate electrospray ionization mass spectrometry (ESI-MS) methods for the detection and identification of fertilizer-based improvised explosives, a non-aqueous solvent mixture was used to preserve the UN and AN ion pairs and discriminate between these and other, similar salts. Direct infusion of standards was first performed and two specific adduct ions were detected for each explosive. Negativeion electrospray ionization was used for UN detection and positive-ion electrospray ionization was used for AN. The specificity of the analysis was tested by mixing the target compounds with various salts and interferents. Adduct ions formed in the gas phase were examined to determine their utility in distinguishing between ion pairs and mixed salts.

In both projects, method validation was performed. These techniques were designed so that they could be of use in practicing forensic laboratories. Further, they were developed with an overall goal of adding useful procedures to those available for forensic scientists.

The final goal of this research is to develop analytical methods for explosives which could be used in the field. A paper microfluidic device for the analysis of fertilizer-based 
explosive mixtures and pyrotechnic compositions was designed and developed. The procedure involved adapting currently available colorimetric tests to permit sensing a variety of explosives simultaneously on chromatographic paper. The system permitted multiplex analysis by incorporating multiple sensing wells defined on the paper through the use of hydrophobic wax barriers. The devices were prepared and tested on mixtures of explosives in soils and other matrices.

\section{References}

1. GlobalSecurity.org. Improvised Explosive Devices (IEDs)/Booby Traps. http://www.globalsecurity.org/military/intro/ied.htm (accessed 12 December 2014).

2. Department of Defense Joint Publication 1-02. 2011. Dictionary of military and associated terms, improvised explosive device. Washington, DC: US Department of Defense. http://www.dtic.mil/doctrine/dod_dictionary/ (accessed 12 December 2014).

3. Davis, T. L. The Chemistry of Powder and Explosives; Angriff Press: Las Vegas, NV, 1943; p. 32.

4. Partington, J.R. A History of Greek Fire and Gunpowder; W. Heffer and Sons Ltd.: Cambridge, U.K., 1960; p.6.

5. Gussman, N. J. Chem. Prog. 2005, 101(4), 64.

6. Davies, R. Hellburner Hoop. Standing Well Back: IED and EOD Evolutions, 2011. http://www.standingwellback.com/home/2011/9/16/hellburner-hoop.html (accessed 8 May 2014).

7. Davies, R. History Repeating Itself. Standing Well Back: IED and EOD Evolutions, 2011. http://www.standingwellback.com/home/2011/9/9/history-repeatingitself.html (accessed 8 May 2014).

8. Mallet, N.H. The Coal Torpedo - The Confederacy's Own Improvised Explosive Device. Military History Now, 2014. http://militaryhistorynow.com/2014/01/13/dirty-trick-the-confederacys-top-secretcoal-torpedo/ (accessed 21 July 2014).

9. Davis, p. 197. 
10. Davies, R. X-raying IEDs - in the 1890s. Standing Well Back: IED and EOD Evolutions, 2011. http://www.standingwellback.com/home/2011/10/21/x-rayingieds-in-the-1890s.html (accessed 11 May 2014).

11. Davis, M. Buda's Wagon: A Brief History of the Car Bomb; Verso/New Left Books: New York, 2007: pp. $1-3$.

12. Webb, S. Dynamite, Treason, and Plot: Terrorism in Victorian and Edwardian London; The History Press: Gloucestershire, U.K., 2012: pp. 49 - 53.

13. Davies, R. 1948 Truck Bombs by British Army Deserters. Standing Well Back: IED and EOD Evolutions, 2014.

http://www.standingwellback.com/home/2014/8/10/1948-truck-bombs-by-britisharmy-deserters.html (accessed 24 November 2014).

14. Roberts, A., Knight, L. By the numbers: Memorial Day and Veterans. CNN.com, 2013. http://www.cnn.com/2012/05/25/politics/numbers-veterans-memorial-day/ (accessed 12 August 2014).

15. Zoroya, G. How the IED Changed the US Military. USAToday.com, 2013. http://www.usatoday.com/story/news/nation/2013/12/18/ied-10-years-blast-woundsamputations/3803017/ (accessed 2 January 2015).

16. StrategyWorld.com. Afghanistan Bans Explosive Fertilizer. http://www.strategypage.com/htmw/htweap/20100125.aspx (accessed 11 May 2011).

17. Davis, p. 1.

18. Mohanty, B. In Forensic Investigation of Explosives, $2^{\text {nd }}$ ed.; Beveridge, A., Ed.; CRC Press: Boca Raton, FL, 2012; pp 24.- 25

19. Yinon, J., Zitrin, S. The Analysis of Explosives; Belcher, R., Betteridge, D., Meites, L., Eds.; Pergamon Series in Analytical Chemistry, Volume 3; Pergamon Press: Oxford, UK, 1981.

20. Explosive Products Division, E. I. DuPont de Nemours and Company, Blasters' Handbook, $16^{\text {th }}$ Edition; Wilmington, DE, 1980, 33.

21. Yeager, K. Improvised Explosives Threat Card. FBI Bomb Data Center Investigator's Bulletin 2006 - 4, and accompanying Threat Card IB 2006 - 4.1.

22. Stine, G.Y. Anal. Chem. 1991, 63(8), 475A.

23. Wissinger, C.E., McCord, B.R. J. Forensic Sci. 2002, 47(1), 168.

24. Heramb, R.B., McCord, B.R. Forensic Sci. Commun. 2002, 4(2), http://www.fbi.gov/hq/lab/fsc/backissu/april2002/mccord.htm (accessed 16 October 2009). 
25. Cascio, O., Trettene, M., Bortolotti, F., Milana, G., Tagliaro, F. Electrophoresis 2004, 25(10 - 11), 1543.

26. Joshi, M., Rigsby, K., Almirall, J.R. Forensic Sci. Int. 2011, 208(1 - 3), 29.

27. Scherperel, G., Reid, G., Waddell Smith, R. Anal. Bioanal. Chem. 2009, 394(8), 2019.

28. Tong, Y., Wu, Z., Yang, C., Yu, J., Zhang, X., Yang, S., Deng, X., Xu, Y., Wen, Y. Analyst 2001, 126, 480.

29. Mathis, J.A., McCord, B.R. J. Chromatogr. A 2003, 988, 107.

30. DuPont, p. 59.

31. Sapir, G.I., Giangrande, M.G. In Aspects of Explosives Detection; Marshall M., Oxley, J.C., Eds.; Elsevier: Oxford, UK, 2009.

32. NDTV, Delhi court blast: 11 dead, 74 injured; sketches of subject released. http://ndtv.com/article/india/delhi-court-blast-11-dead-74-injured-sketches-ofsubject-released132067 (accessed 18 August 2012).

33. Almog, J., Burda, G., Shloosh, Y., Abramovich-Bar, S., Wolf, E., Tamiri, T. J. Forensic Sci., 2007, 52, 128.

34. Woodfin, R.L. Trace Chemical Sensing of Explosives; John Wiley \& Sons Inc., Hoboken, NJ, 2007.

35. StrategyWorld.com, accessed 11 May 2011.

36. aware eZine Gamma, Improvised Urea Nitrate. http://www.awarenetwork.org/etc/gamma/?x=5 (accessed 15 June 2012).

37. ShadowRx Forums, Urea Nitrate. http://www.shadowrx.com/forums/showthread.php?t=976 (accessed 15 June 2012).

38. Yeager, K. In Forensic Investigation of Explosives, $2^{\text {nd }}$ ed.; Beveridge, A., Ed.; CRC Press: Boca Raton, FL, 2012; pp 504 - 507.

39. Perez, J. J., Flanigan, P.M., Brady, J. J., Levis, R. J. Anal. Chem., 2013, 85, 296.

40. Lopez-Lopez, M., Carlos Bravo, J., Garcia-Ruiz, C. Torre, M. Talanta, 2013, 103, 214.

41. Meng, H-H., Caddy, B. J. Forensic Sci. 2001, 46, 549.

42. Stankovic, M., Vujovic, B., Filipovi, M. Chromatographia, 1996, 42, 593.

43. Zhao, X., Yinon, J. J. Chromatogr. A, 2002, 977, 59. 
44. Hopper, K., McCord, B. J. Forensic Sci., 2005, 50, 307.

45. Hopper, K. G., LeClair, H., McCord B. Talanta, 2005, 67, 304.

46. Northrop, D. M., Martire, D. E., MacCrehan, W. A. Anal. Chem., 1991, 63, 1038.

47. Smith, K. D., McCord, B.R., MacCrehan, W. A., Mount, K., Rowe, W. F. J. Forensic Sci., 1999, 44, 780.

48. Zhao, X., Yinon, J. Rapid Commun. Mass Spectrom., 2001, 15, 1514.

49. Hall, K., McCord, B.R. J. Forensic Sci., 1993, 38, 928.

50. Almog, J., Klein, A., Tamiri, T., Shloosh, Y., Abramovich-Bar, S. J. Forensic Sci., 2005, 50, 582 .

51. Harkema, S., Feil, D. Acta Cryst. B, 1969, 25, 589.

52. Zitrin, S., Tamiri, T. In Forensic Investigation of Explosives, $2^{\text {nd }}$ ed.; Beveridge, A., Ed.; CRC Press: Boca Raton, FL, 2012; pp 680 - 684.

53. Cagan, A., Lu, D., Cizek, K., La Belle, J., Wang, J. Analyst, 2008, 133, 585.

54. Tamiri, T., Rozin, N., Lemberger, N., Almog, J. Anal. Bioanal. Chem., 2009, 391, 421.

55. de Perre, C., McCord, B. Forensic Sci. Int., 2011, 211, 76.

56. Almog, J., Espino, D., Tamiri, T., Sonenfeld, D. Forensic Sci. Int., 2013, 224, 80.

57. Tamiri, T. Rapid Commun. Mass Spectrom. 2005, 19, 2094.

58. de Perre, C., Prado, A., McCord, B. R. Rapid Commun. Mass Spectrom., 2012, 26, 154.

59. Wynn, C. M., Palmacci, S., Kunz, R. R., Rothschild, M. Opt. Express, 2010, 18, 5399.

60. Almog, J. J. Forensic Sci., 2006, 51, 1228.

61. Yinon, J. TrAC, Trends Anal. Chem., 2002, 21, 292.

62. Li, X., Ballerini, D., Shen, W. Biomicrofluidics, 2012, 6, 011301.

63. Taudte, R., Beavis, A., Wilson-Wilde, L., Roux, C., Doble, P., Blanes, L. Lab Chip, 2013, 13, 4164.

64. Salles, M., Meloni, G., de Araujo, W., Paixao, T. Anal. Methods, 2014, 6, 2047.

65. Pesenti, A., Taudte, R., McCord, B., Doble, P., Roux, C., Blanes, L. Anal. Chem., 2014, 86, 4707. 
66. Transcript of the testimony of Dr Frederic Whitehurst, (S5) 93 Cr. 181, 14 August 1995. http://www.web-ak.com/waco/death/tscr/whitehur/fw test.html, pages 16324 16353. 


\section{CHAPTER 2: Separation and Identification of Organic Components of Smokeless Gunpowder by Capillary Electrochromatography}

\section{A. Introduction}

This section details a method for the separation and identification of fourteen organic compounds commonly found as constituents of commercial smokeless powders using a hexyl acrylate-based porous monolith. Capillary electrochromatography (CEC) coupled to UV and time of flight-mass spectrometry (TOF-MS) methods were both explored. The CEC-UV method provides an effective and efficient method for the detection of all components in the additive package of the powder. The TOF-MS procedure provides better sensitivity and selectivity and allows an additional confirmation of the presence of the subset of those compounds, which are detectable via positive and/or negative ion electrospray ionization mass spectrometry (ESI-MS). Both methods were used for the analysis of smokeless powder components in a mixed standard as well as in the determination of the composition of the additive package of individual powders.

Smokeless gunpowder is commonly used as a propellant for rifle and handgun ammunition. Based on nitrocellulose, it is manufactured worldwide and easily bought in bulk for sportsmen interested in reloading ammunition for such things as hunting and competitive target shooting. Because smokeless powders are so easily purchased, they can readily be diverted for use in improvised explosive devices. Of the pipe bomb incidents reported to the US Bureau of Alcohol, Tobacco, Firearms and Explosives between January 2005 and November 2009, at least 37\% were reported as having smokeless or black powder as the explosive charge. It can be critical to identify the type 
and source of the powder following such an incident in order to determine the perpetrator of the bombing. Luckily, it is common for many unburned flakes of smokeless powder to be thrown clear of the device during the blast and these particles may be characterized if found. Morphology and chemical composition are key factors in the characterization of the various types of powder used in these devices.

Smokeless powders can be classified based on the type of energetic compounds present in their composition. Single base powders contain only nitrocellulose. Double-base powders contain nitrocellulose and nitroglycerin; and triple-base powder contains nitrocellulose, nitroglycerin, and nitroguanidine. Rifle powders typically are single-based powders as their longer muzzle permits the use of less energetic formulations. These powders may also contain dinitrotoluene. Powders used in pistols and other small arms contain nitroglycerine in addition to nitrocellulose to enhance their reaction rate and improve the velocity of projectiles exiting from shorter muzzles. Triple based powders are used in large bore military guns and rockets. In addition to the energetic components, modern smokeless powder formulations include an additive package consisting of stabilizers, plasticizers, flash suppressants, deterrents, opacifiers, and dyes [3].

Stabilizers present in the powder scavenge the nitric and nitrous acids produced by the decomposition of nitrocellulose and nitroglycerin. If left to accumulate, nitric and nitrous acids can catalyze a decomposition reaction in the powder. Interestingly, the concentration and variety of these nitrated stabilizers present in a powder may be used as a measure of its age or lot number $[1,2]$. For example diphenylamine, a common stabilizer, will react to produce a variety of nitro- and nitrosodiphenylamines. 
<smiles>COC(=O)c1ccccc1C(=O)OC</smiles><smiles>CN(C(=O)N(C)c1ccccc1)c1ccccc1</smiles><smiles>CCOC(=O)c1ccccc1C(=O)OCC</smiles><smiles>O=[N+]([O-])OCC(CO[N+](=O)[O-])O[N+](=O)[O-]</smiles><smiles>CCN(C(=O)N(CC)c1ccccc1)c1ccccc1</smiles>

6)<smiles>Cc1ccc([N+](=O)[O-])c([N+](=O)[O-])c1</smiles>

10)<smiles>O=Nc1ccc(Nc2ccccc2)cc1</smiles>

13)

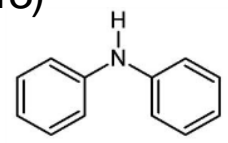

14)<smiles>O=[N+]([O-])c1ccccc1Nc1ccccc1</smiles>

7)<smiles>Cc1ccc([N+](=O)[O-])cc1[N+](=O)[O-]</smiles>

11)<smiles>CCCCCOC(=O)c1ccccc1C(=O)OCCCC</smiles>

8)<smiles>Cc1c([N+](=O)[O-])cccc1[N+](=O)[O-]</smiles>

12)<smiles>O=[N+]([O-])c1ccc(Nc2ccccc2)cc1</smiles>

Figure 2-1. Smokeless powder additives analyzed in this study: 1) dimethylphthalate (DMP), 2) methyl centralite (MC), 3) diethylphthalate (DEP), 4) nitroglycerin (NG), 5) ethyl centralite (EC), 6) 3,4dinitrotoluene (3,4 DNT), 7) 2,4-dinitrotoluene (2,4 DNT), 8) 2,6-dinitrotoluene (2,6 DNT), 9) 4nitrosodiphenylamine (4-NsDPA), 10) N-nitrosodiphenylamine (N-NsDPA), 11) dibutylphthalate (DBP), 12) 4-nitrodiphenylamine (4-NDPA), 13) diphenylamine (DPA), 14) 2-nitrodiphenylamine (2-NDPA).

As a result, determining the identity and concentration of these products is an important component of any analytical or forensic assay (Table 2-1). Other common stabilizers include methyl and ethyl centralites (alkyl substituted dinitrodiphenylureas) [3].

Plasticizers reduce the need for volatile solvents during the formation and extrusion of the powder. A variety of alkyl substituted phthalates are common in these powders, 
including methyl, ethyl and butyl phthalates. Nitroglycerin, while mainly useful as the primary energetic material in smokeless powder, can also function as a plasticizer. Alkali or alkaline earth metal salts function as flash suppressants, reducing muzzle flash when a weapon is fired. Deterrents and opacifiers are surface coatings that modulate the burn rate of the individual grains and enhance the reproducibility and efficiency of the deflagration rate of the powder. Lastly dyes are sometimes added to assist in the visual identification of some brands of powder [3]. The variety of materials present in the additive package can vary widely, depending on the manufacturer's desired combustion rate and formulation.

In addition to the additive package, smokeless powders can be characterized based in their morphology. A variety of manufacturing processes exist, including methods of extrusion and precipitation [3]. These different processes create morphologies that include balls, discs, rods, tubes, lamels and various aggregates. Combining morphology with chemistry helps to individualize classes and manufacturers of the different powders.

Given the implications for law enforcement and national security organizations, considerable work has been devoted to the identification of smokeless powders by characterizing these components present in the additive package [4-8]. The ultimate goal is to identify the source of the smokeless powder found at a bombing scene by detecting the number and type of additives used in the manufacture of the powder.

Characterization of age or lot number may also be possible in certain situations. In addition, the presence of smokeless powders on skin or clothing can be used as an indication that that individual has been in the presence of a fired weapon. Many different 
procedures have been developed for the determination of organic components of smokeless powder, and thorough reviews have been done by Meng and Caddy [9] and Goudsmits et. al [10]. Because of the importance of proper identity of the components in the powder, chromatographic methods are commonly used for these samples.

For example, gas chromatography coupled to flame ionization detection (FID) or mass spectrometry (MS) has been used for characterization of smokeless powders but requires tight control of injector temperature. Otherwise, nitrated organic compounds such as nitroglycerin and nitrosodiphenyl amine may undergo thermal decomposition in the heated zones of the gas chromatograph [11]. To overcome the limitations imposed by gas chromatography, high performance liquid chromatography (HPLC) and HPLC-MS techniques have also been developed [8,12]. A significant amount of research has also been performed using capillary electrophoresis procedures $[13,14]$. However, since free zone capillary electrophoresis techniques cannot separate neutral molecules present in a sample, micellar electrokinetic chromatography (MEKC) techniques were developed $[15,16]$. MEKC based procedures can provide enhanced resolution of isomeric nitrotoluenes and nitrated diphenyl amines when compared to liquid chromatography. In a series of papers, the research group of MacCrehan and Northrop demonstrated that capillary electrophoresis in the MEKC mode is an effective method for the analysis of these components in smokeless powders [15-17].

Unfortunately, coupling MEKC to mass spectrometers is awkward because of the use of non volatile surfactants in the buffer. There have been a few reports in the literature discussing the interfacing of MEKC based methods to mass spectrometry using partial 
filling techniques, complex control of solute charges, $\mathrm{pH}$, and wall effects; however, such techniques may be difficult to implement on a regular basis $[18,19]$.

Capillary electrochromatography (CEC) is a hybrid of liquid chromatography and capillary electrophoresis that uses a fused silica capillary with a diameter of 50-100 $\mu \mathrm{m}$ that has been filled with a stationary phase. Because of embedded charges in the capillary wall and on the stationary phase, the mobile phase can be electroosmotically driven with lower diffusion than is found in pressure driven systems [20].

The partitioning of the analytes between the mobile phase and stationary phases as well as differences in electrophoretic mobilities result in highly efficient separations. CEC has been applied to the study of explosives using both packed columns [21] and sol-gel stationary phases [22], but the explosives analyzed by the technique have so far been limited to high explosives such as nitroaromatics and nitramines.

\section{B. Goals of This Project}

The goal of this project was to develop a method for the analysis of smokeless powders using monolithic CEC in a manner that would be compatible with MS detection. In addition, we were interested in determining if a monolithic CEC system could provide the high resolution necessary to separate geometric isomers of dinitrotoluene and nitrodiphenyl amine, which can aid in the determination of lot to lot variations in the composition of powders. MacCrehan and Northrop showed that MEKC is an effective method for analysis of geometric isomers of dinitrotoluene and nitrodiphenyl amine in smokeless powders [15 - 17]. As previously mentioned, though, MEKC is not compatible 
with mass spectrometry. Interfacing MEKC methods to mass spectrometry has been discussed in the literature using such techniques as countercharged cyclodextrins or partial filling techniques, which may be difficult to implement [23, 24]. The inherent difficulties in these techniques lead to the consideration of CEC-MS as an analytical tool for these samples. A number of advantages present themselves for smokeless powder samples: the high resolution of this technique allows the separation of geometric isomers such as dinitrotoluenes and nitrodiphenyl amines, which aid in the determination of lotto-lot variations in the composition of smokeless powder, and CEC methods may permit preconcentration of the sample, improving the sensitivity of the assay [25].

\section{Fundamentals of Capillary Electrochromatography}

Capillary electrochromatography (CEC) is a hybrid technique that combines aspects of HPLC and capillary electrophoresis (CE). CEC uses a fused silica column with a diameter of $50-100 \mu \mathrm{m}$ that contains a stationary phase, and an electric field is applied across the column (Figure 2-2). The electroosmotic flow $u_{\mathrm{eo}}(\mathrm{EOF})$ that is generated, rather than an applied pressure, moves the mobile phase and analytes through the column. CEC differs from micellar electrokinetic chromatography (MEKC) in that it uses a solid stationary phase to generate the separation instead of micelles moving through a liquid buffer under the influence of an electric field. In CEC, sample components separate because of both the partitioning effect of the stationary phase and the electrophoretic mobility, $u_{\mathrm{ep}}$, of the compounds. CEC also differs from MEKC in that the analytes interact with the stationary phase so neutral analytes can also be separated. Due to the embedded charges in the capillary wall and the stationary phase, the mobile phase is 
electroosmotically driven, limiting the dispersion seen with pressure driven laminar flow. Electroosmotic flow minimizes band broadening as the sample moves through the column. CEC has been applied to the study of explosives using packed columns [21] and sol-gel columns [22] for nitroaromatic and nitramine explosives.

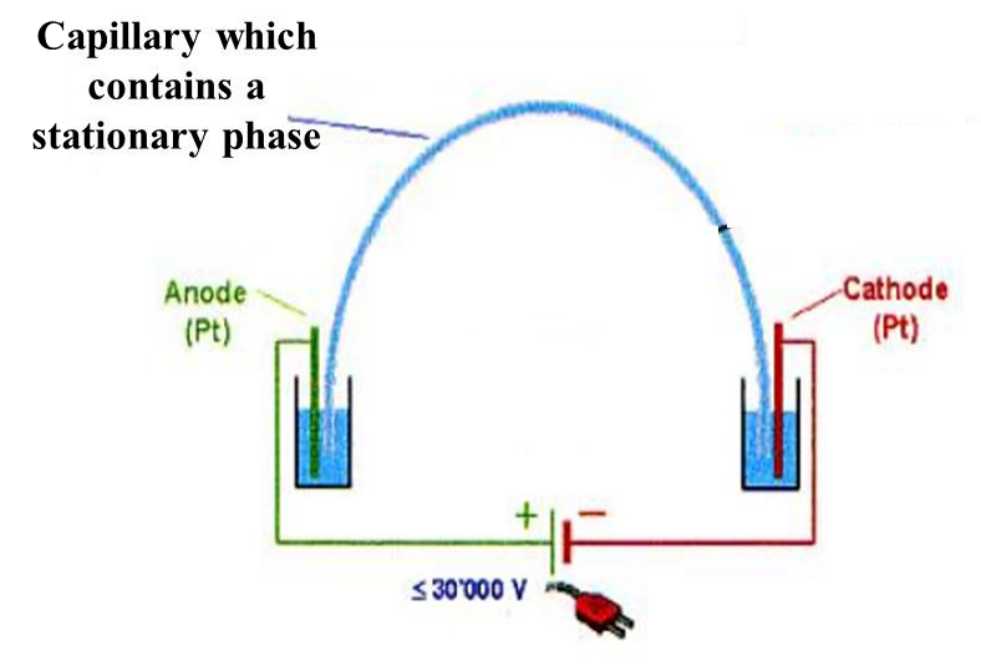

Figure 2-2. Simple schematic of a CEC instrument.

\section{Instrumentation}

\section{a. Electroosmotic Flow (EOF)}

Electroosmotic flow (EOF) occurs because of the presence of an electrical double layer (EDL) at the interface of the liquid mobile phase and the ionized surface of the stationary phase. The EDL is composed of two parts. When an electric field is applied, the negative charges on the stationary phase remain fixed and are balanced by positive charges in the mobile phase. The positive charge layer closest to the capillary wall, called the Stern layer, is adsorbed onto the stationary phase and effectively immobile. Next to the Stern layer is the shear layer, where charges are almost immobile. The diffuse layer, next to the 
shear layer, is not as highly ordered and under the influence of the electric field the ions migrate parallel to the EDL to the corresponding electrode, pulling the mobile phase with it and generating bulk liquid flow (Figure 2-3). The mobile phase and solutes are pulled along at a constant rate so analytes move through the column with a uniform flow profile that is not subject to the band broadening that occurs with the pressure-induced flow of gas or liquid chromatography (Figure 2-4) [26]. Column efficiency is increased and with no pressure gradient, longer columns with more dense packing can be used.

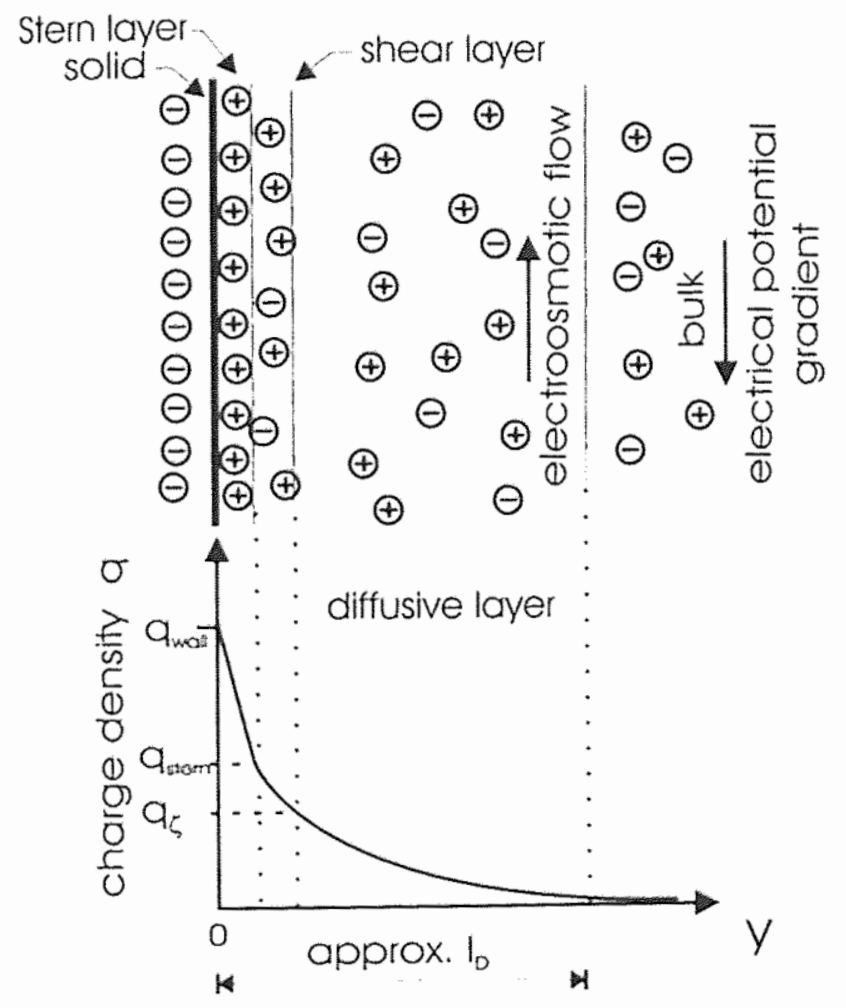

Figure 2-3: Graphic of the electrical double layer and distribution of charge [Barz, 26] 


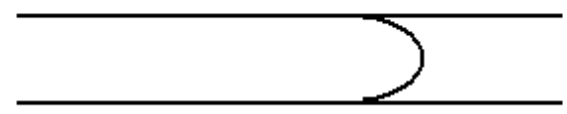

Laminar flow

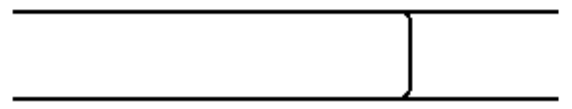

Electroosmotic flow

Figure 2-4: Flow profile comparison of laminar and electroosmotic flow.

Neutral compounds move with the EOF and are separated by their interaction with the mobile and stationary phases. Electrophoretic migration occurs for charged compounds. Depending on a compound's charge, it can move with or against EOF, so electrophoretic mobility contributes to selectivity in a CEC separation. The linear velocity of the EOF, $u_{\mathrm{eo}}$, is described by Equation 1:

$$
u_{\mathrm{eo}}=\mu_{\mathrm{eo}} E=\frac{\varepsilon_{o}-\varepsilon_{r} \zeta}{\eta} E
$$

where

$\varepsilon_{\mathrm{o}}$ is the permittivity of vacuum

$\varepsilon_{\mathrm{r}}$ is the dielectric constant (relative permittivity)

$\zeta$ is the zeta potential at the shear surface

$\eta$ is the viscosity of the mobile phase

$\mathrm{E}$ is the strength of the applied electric field

The sample vials may be pressurized slightly (10 bar) to avoid bubble formation in the column. The additional pressure also accelerates the separation but has the potential to change the flow profile. Taking all these factors into account, the overall migration velocity is expressed in Equation 2: 


$$
u_{o b s}=\frac{\left(u_{e o}+u_{e p}+u_{p r e s s}\right)}{(1+k)}
$$

There are a number of other factors that must be taken into account when "tuning" the EOF to control surface charges when designing a CEC method.

\section{b. Stationary Phase}

The stationary phase generates EOF and provides chromatographic selectivity. EOF velocity is proportional to the zeta potential of the stationary phase, which depends on the charge density at the surface of the stationary phase. The zeta potential shows the stability of the monolith; higher zeta potentials are a characteristic of more stable systems. When the zeta potential is low, the system becomes unstable and EOF is weak or nonexistent.

\section{c. Voltage Effects and Joule Heating}

Applied voltage drives the separation in CEC. It is responsible for the speed of the separation and is usually between 10 and $30 \mathrm{kV}$ in commercial instruments. This limit is imposed because of the effects of Joule heating on the capillary. Joule heating, also called resistive heating, occurs when the electrical resistance of the capillary dissipates the applied power, causing the system to heat up. If the heat that is generated cannot be removed from the system, EOF and retention time can change causing detrimental effects on the efficiency and reproducibility of the system. If Joule heating can be controlled, higher run voltages are better for separation but current technology limits stable power supplies to a maximum of $30 \mathrm{kV}$ [27]. 
Increasing the voltage increases the flowrate, which decreases the runtime. Doubling the voltage cuts the runtime in half; tripling the voltage decreases runtime by $2 / 3$. At the optimum voltage, the reduced plate height $\mathrm{h}$ is minimized and resolution, $\mathrm{R}_{\mathrm{s}}$, is increased but applied voltage has minimal effect on capacity factor, $k^{\prime}$ [20].

\section{d. Ionic Strength}

Since constant voltages are applied, the conductivity of the mobile phase in CEC affects the current. As buffer concentration increases, EOF decreases, causing longer run times and potential changes in elution order. To avoid reducing EOF, buffers are made as dilute as possible without negatively affecting results.

EOF is reduced at higher buffer concentrations because ionic strength also affects the double layer inside the column. The double layer is the transition from the ordered Stern layer that balances the charges on the wall of the silica capillary to the random charge arrangement in the bulk solution. As the ionic strength of the buffer increases, the thickness of the double layer decreases and EOF is compromised [28]. There are also pH considerations in CEC. If the $\mathrm{pH}$ of the mobile phase is too low (less than 3 ) the silanol groups in the monolith are protonated and EOF comes to a halt. At a $\mathrm{pH}$ of greater than 8 , silica columns dissolve and monolithic separation media may become detached from the capillary wall [20].

\section{e. System Temperature}

The effect of temperature on an LC system is minimal, but temperature variations can have an effect on the reproducibility of results in CEC systems. As with other types of 
chromatography, increasing the temperature at which a sample is run decreases the run time. A CEC column is generally held about $5^{\circ} \mathrm{C}$ above room temperature. Higher temperatures can cause bubble formation in the mobile phase, although the tendency for bubbles to form can be controlled somewhat by applying about 10 bar of pressure to both vials. Changes in temperature cause changes in the viscosity of the mobile phase, which affects electrophoretic mobility. Since the viscosity term, $\eta$, is in the denominator of Equation 1, a small change in the value of $\eta$ can have a large effect on electroosmotic mobility.

\section{f. Mobile Phase Composition}

As in HPLC, the composition of the mobile phase influences the partitioning of sample between the mobile and stationary phases. The composition of the mobile phase affects the runtime and elution order, so it depends on the chromatographic system and the type of sample being separated. If a high proportion of acetonitrile (ACN) is used in the mobile phase, runtime is shortened but EOF is decreased. If the proportion of ACN is too high, the current may take 30 minutes or longer to stabilize at a constant voltage [20].

Unlike HPLC, the mobile phase in CEC is not static. During the elution process, hydrogen ions and hydroxide ions produced by the electrolytic process change the $\mathrm{pH}$ of the sample buffer, which in turn affects sample loading behavior.

\section{g. Capillary Considerations}

A capillary that is too short gives bad resolution because there are an insufficient number of theoretical plates for the sample components to separate. If the capillary is too long, 
the time needed to run a sample is too long also, but separation efficiency is increased because the uniform, pluglike flow associated with EOF is not subject to band broadening as pressure-driven flow is. The ends of the capillary must be cut perfectly square, or else peak shape is affected and electrokinetic sample injections become irreproducible [20].

\section{Electrospray Ionization}

Electrospray ionization (ESI) is a soft ionization technique that creates ions in solution at atmospheric pressure. Both negatively and positively charged ions can be produced. Analytes undergo a minimum amount of fragmentation and weak ionic associations are preserved with this ionization technique. ESI is used for small and large molecules. The quasimolecular ion takes the form of $[\mathrm{M}+\mathrm{H}]^{+}$in positive ion mode and $[\mathrm{M}-\mathrm{H}]^{-}$in negative ion mode, or as a charged adduct that forms in conjunction with the ionization promoter in the mobile phase. It was demonstrated as a sample ionization technique by Malcolm Dole in 1968 [29]. In the electrospray process, compounds in solution are transferred to the gas phase as ions for analysis by mass spectrometry. ESI is used on a wide variety of molecules including proteins, polymers, pollutants, and drugs. The ESI process generates positively or negatively charged ions. Neutral compounds may acquire a charge by clustering with adduct ions. Charging of the analyte is part of the electrospray process, which occurs in three steps.

\section{Production of Charged Droplets}

The production of charged droplets occurs at the capillary tip. A voltage of $2-4 \mathrm{kV}$ is applied to the steel capillary through which the sample passes, creating a potential 
between the capillary and the counter-electrode. The surface of the liquid at the end of the capillary is charged and breaks up to form a spray of charged droplets.

The spray is formed when the applied voltage overcomes the surface tension of the liquid. The drop at the tip of the steel capillary elongates under the pressure of the charges that accumulate. At the appropriate voltage the surface tension is broken, the shape of the liquid at the end of the capillary becomes conical, and a jet is emitted from the tip of the cone. The jet breaks up into droplets which further disintegrate to form the plume of ionized sample. Nitrogen gas is used to nebulize the liquid and help evaporate the solvent.

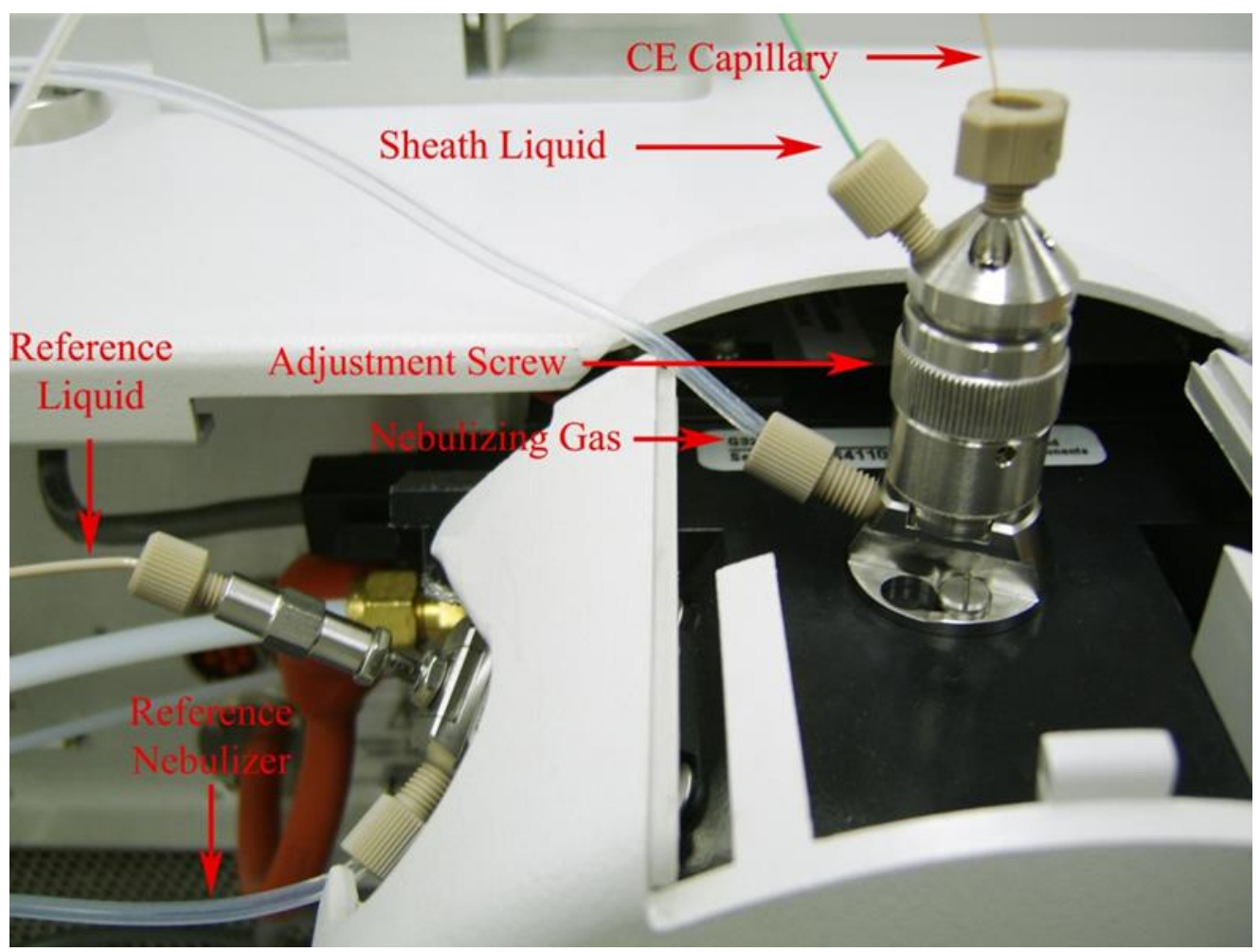

Figure 2-5. Closeup of CEC-MS interface. 
The droplets are charged because of an excess of electrolyte ions at the surface of the cone. After the droplets are formed, they move through the electrospray chamber toward the first vacuum stage of the mass spectrometer. Solvent evaporation is assisted by nitrogen gas used to nebulize the liquid, shrinking the droplets and increasing the surface charge (Figure 2-5).

\section{Production of Gas Phase Ions}

When the drop becomes small enough, it undergoes a process called Coulomb fission, where the repulsive force between the charges overcomes the surface tension of the droplet and it "explodes," creating many smaller, more stable droplets which undergo the same process until all the solvent is evaporated and the gas-phase ion is left. There are two competing theories about the mechanism of formation for gas-phase ions in electrospray: the ion evaporation model and the charged residue model.

\section{a. The Ion Evaporation Model}

In the ion-evaporation model proposed by Iribarne and Thompson [30], the increasing electric field on the surface of the droplet causes solvent-ion clusters to separate and desorb from the surface. Repulsion between like charges overcomes the surface tension of the droplet and direct ion emission becomes possible. This process dominates over Coulomb fission when the radius of the droplets is $\leq 10 \mathrm{~nm}$ [31].

\section{b. The Charged Residue Model}

In the charged residue model proposed by Malcolm Dole, gas-phase ions form as a result of repeated droplet fission that happens as the droplets shrink through solvent 
evaporation, leaving the charged ion to move into the low-vacuum area of the mass spectrometer. This mechanism is considered to be the more likely path for large molecules such as proteins [31].

\section{Time-of-Flight Mass Spectrometry (TOF-MS)}

The purpose of a mass spectrometer is to determine the mass of a charged particle. After being ionized the charged particle is pulled into the mass spectrometer, where its mass is measured. Inside the mass spectrometer, the ions are sorted by their mass-to-charge $(\mathrm{m} / \mathrm{z})$ ratios and detected according to their abundance, producing the mass spectrum of the molecule as either a graph showing the ions and their abundance, or as a table.

There are two broad types of mass analyzer, sequential or simultaneous. The magnetic sector mass spectrometer is the original example of a sequential mass analyzer, but the most common is the quadrupole analyzer. Composed of four rods set in a parallel arrangement, it is the most common type of mass analyzer used for the identification of explosives and related compounds. It can be coupled to an electron ionization (EI), chemical ionization (CI), or atmospheric pressure source such as ESI or atmospheric pressure chemical ionization (APCI). It separates the ions using a combination of electric and magnetic fields, allowing only ions with the correct $\mathrm{m} / \mathrm{z}$ value to reach the detector. The quadrupole mass spectrometer may be coupled to a GC or LC which separates the compounds before moving them into the mass spectrometer.

Simultaneous mass analyzers include the various configurations of the ion trap, ion cyclotron resonance instruments, and the time-of-flight mass spectrometer (TOF-MS). 
Rather than scanning a stream of ions, this type of instrument allows the simultaneous transmission of all ions. After the ions are formed as described above in the first vacuum stage of the instrument, the fragmented ions pass through the skimmer aperture as nebulizing gas is deflected by the skimmer and drawn out by a rough pump.

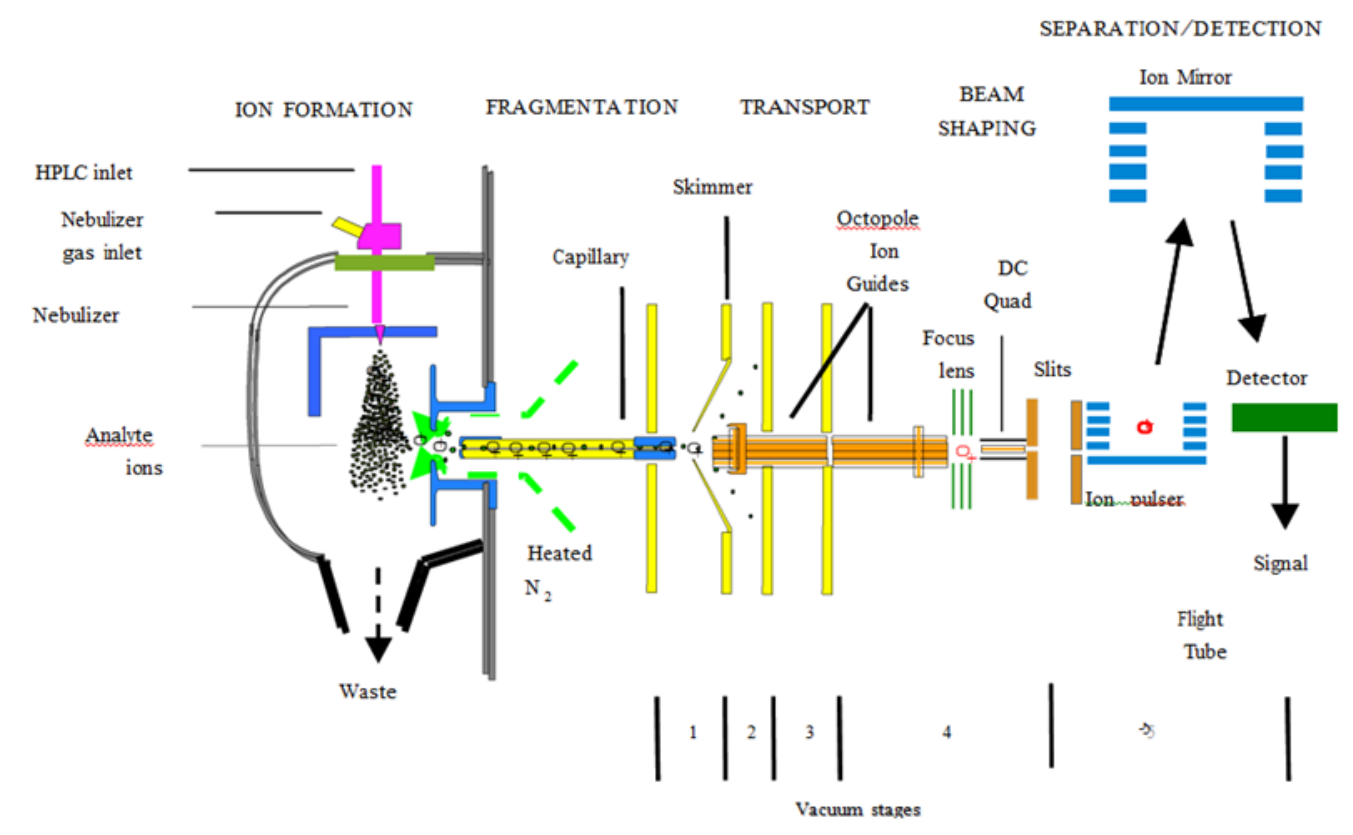

Figure 2-6. Schematic of ESI-TOF-MS instrument [35].

In the second vacuum stage the ions are focused by an octopole. Ions above a set mass range are filtered out and the remaining ions pass into the third vacuum stage, where a second octopole accelerates the ions into the fourth vacuum stage.

In the fourth vacuum stage, lenses focus the ions into a parallel beam before they enter the time-of-flight analyzer. The resolution of the mass spectrum produced by the TOF 
depends on the ions in the beam moving in parallel when they enter the fifth vacuum stage, where separation takes place.

In the final stage the ion beam passes into the TOF's ion pulser from the side. The ion pulser is a stack of plates, all of which have a center hole except the back plate. A high voltage pulse is applied to the back plate, accelerating the ions out of the pulser and into the flight tube. At the opposite end of the flight tube, the ion mirror reverses the direction of the ions and directs them to the detector. The ion mirror is composed of a stack of metal plates to which a voltage is applied.

All ions acquire the same amount of energy when they leave the pulser. The separation occurs based on the size of the ions. As the ions move away from the pulser into the evacuated flight tube, smaller ions, or those with a lower $\mathrm{m} / \mathrm{z}$ ratio, will move more quickly than larger ions. At the far end of the flight tube is the reflectron, an electrostatic reflector made up of a series of ring electrodes that corrects the kinetic energy dispersion of the ions leaving the source. Ions leave the source and move down the flight tube. As they penetrate the reflectron field at the other end of the flight tube their momentum is reversed and the ions move back down the flight tube to the detector (Figure 2-7). 


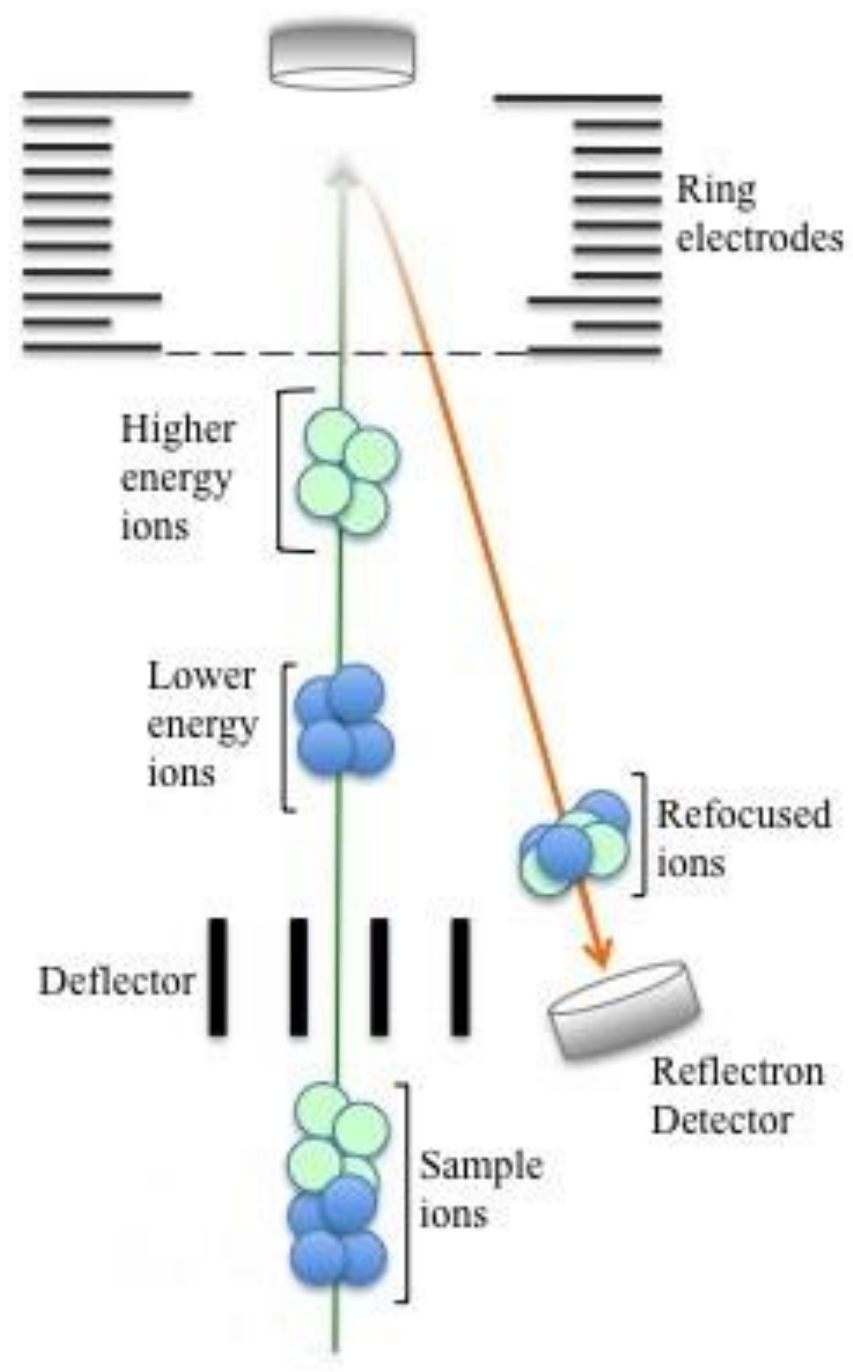

Figure 2-7. The reflectron corrects the kinetic energy dispersion of ions as they leave the source.

If two ions have the same mass but different kinetic energies, the ion with a higher KE will also have a higher velocity and penetrate farther into the reflectron than those with less kinetic energy. Ions with less kinetic energy reach the reflectron later but do not penetrate as deeply, so ions with the same mass leave at the same time and strike the detector at the same time. [32] The reflectron serves as a corrector for the variations in flight times of ions of the same mass. 


\section{E. Materials and Methods}

\section{Reagents and Materials}

Fused silica capillaries with a $75 \mu \mathrm{m}$ inner diameter and a UV-transparent coating were purchased from Polymicro Technologies (Phoenix, AZ). Hexyl acrylate (HA), 1,3butanediol diacrylate (BDDA), 2-acrylamido-2-methyl-1-propanesulfonic acid (AMPS), 2,2'-azobisisobutyronitrile (AIBN), trimethoxysilylpropyl acrylate, and smokeless powder additive standards (Figure 1) were purchased from Aldrich Chemical (St. Louis, MO). Diphenylamine standard was purchased from Acros Organics (Morris Plains, NJ).

\section{a. Monolith Preparation}

Monolithic columns were prepared following a method adapted from Ngola [33]. A polymerization solution was prepared using $1379 \mu \mathrm{l}$ hexyl acrylate (HA) and $591 \mu \mathrm{l}$ 1,3-BDDA. To this was added $0.0145 \mathrm{~g}$ AMPS and $4 \mu 1$ trimethoxysilylpropyl acrylate. A sodium phosphate buffer was made from a solution containing $3 \mathrm{mM}$ sodium dihydrogen phosphate and $4 \mathrm{mM}$ disodium hydrogen phosphate, adjusted to $\mathrm{pH} 6.8$ with a $1.0 \mathrm{M}$ sodium hydroxide solution. The porogenic mixture was composed of 3 parts by volume acetonitrile, 1 part by volume ethanol, and 1 part by volume sodium phosphate buffer. The monomer mixture was mixed with the porogen in a $1: 2$ ratio and $9.4 \mathrm{mg}$ of the radical photoinitiator AIBN were added to the solution. The mixture was sonicated to remove dissolved air, leaving a clear solution.

The capillary was pretreated by flushing with acetone for $10 \mathrm{~min}$ and then with $1.0 \mathrm{M}$ sodium hydroxide for $1 \mathrm{~h}$ before treating the inner wall with $20 \mu \mathrm{l}$ trimethoxysilylpropyl 
acrylate in $1 \mathrm{ml} 6 \mathrm{mM}$ acetic acid for one hour. The capillary was rinsed with deionized water for 30 minutes and dried with a flow of nitrogen gas [34]. One end of the capillary was immersed in the polymerization solution, which was pushed through the capillary using pressurized nitrogen.

After filling, the ends of the capillary were sealed and a $1 \mathrm{~cm}$-wide piece of aluminum foil was wrapped around the capillary at the location of the UV window. The prepared capillary was placed under a $365 \mathrm{~nm}$ UV lamp (UVP, Upland, CA) for $1 \mathrm{~h}$ at room temperature to initiate polymerization. After curing, the capillary was cut to the proper length and installed into the CE instrument (Agilent, Santa Clara, CA). To remove residual monomer and porogen before CEC-UV analysis, a gradually increasing voltage from 0 to $30 \mathrm{kV}$ was applied to the capillary for $1 \mathrm{~h}$, then the voltage was kept at $30 \mathrm{kV}$ for $5 \mathrm{~h}$ (or until the baseline was stable) using an $80 \%$ ACN-20\% phosphate buffer eluent. Both ends of the capillary were pressurized with 10 bar of nitrogen gas to avoid bubble formation in the column during this step. When switching to CEC-MS, an additional short conditioning was performed with a gradual voltage from 0 to $30 \mathrm{kV}$ for $30 \mathrm{~min}$ and then held at $30 \mathrm{kV}$ until the current was stable. A pressure of 2 bar was applied to the inlet side of the capillary during this step. The column was conditioned on a daily basis prior to injection of samples by passing a freshly prepared eluent made of $75 \%$ ACN $25 \%$ phosphate buffer through the column until the current and the baseline were stable.

\section{b. Monolith Examination}

Even after the proper polymerization solution formula was determined, not all monolith fabrication attempts were successful. After adding the porogenic solvent to the 
polymerization solution, the mixture was agitated to mix the two layers. When properly made, the result was a clear, colorless liquid which, if left to sit in the freezer, would separate into two clear, colorless layers. The mixture would sometimes take on a cloudy appearance instead of remaining clear during both mixture and separation. Monoliths made with the cloudy mixture did not polymerize well and repeatability values for samples run using these monoliths were unstable. Examples of successful and unsuccessful monoliths were examined by scanning electron microscopy in an attempt to see if there were any differences between successful and unsuccessful monoliths.

a.

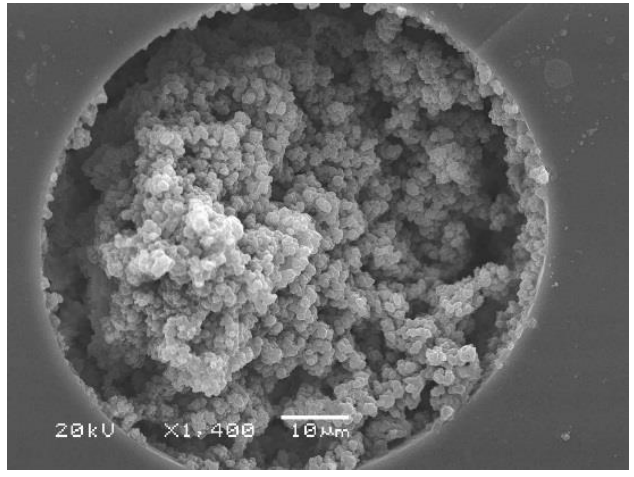

c.

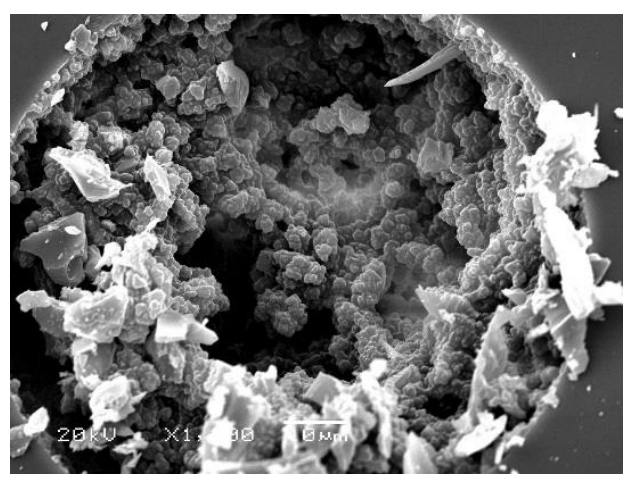

b.

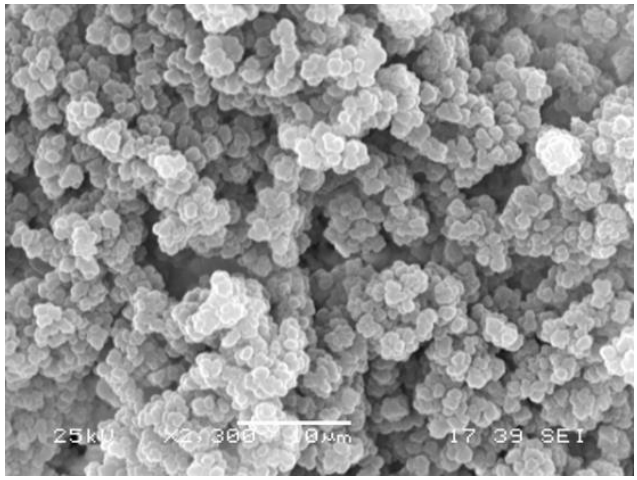

d.

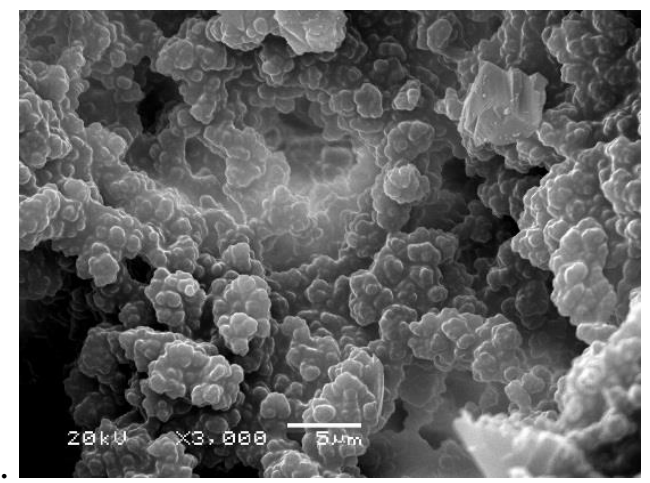

Figure 2-8. Scanning electron microscopy images of a.) successful monolith showing uniform polymerization; b.) closeup image of successful monolith; c.) unsuccessful monolith showing nonuniform polymerization; d.) closeup image of unsuccessful monolith.

Polymerization in the successful monoliths is uniform and shows good adhesion to the capillary wall (Figure 2-8). In contrast, the polymerization in unsuccessful monoliths was 
uneven, leaving large "holes" in the monolith where growth of the polymer did not occur. The monolith also did not adhere to the capillary wall properly. As an aside, it was noted that if the polymerization solution was mixed on days of extremely high South Florida humidity the solution was more likely to be cloudy and the monolith made from the cloudy solution was more likely to fail. The freezer test became a quick way to determine whether a monomer mixture would produce a usable capillary.

\section{Instrumentation}

Separation of the smokeless powder standards and samples was performed using an Agilent G1600AX CE instrument with the column temperature maintained at $25^{\circ} \mathrm{C}$. The capillary length was $50 \mathrm{~cm}$ and the run voltage was $30 \mathrm{kV}$. Samples were injected using electrokinetic injection for $8 \mathrm{~s}$ at $10 \mathrm{kV}$. UV detection was performed using a Diode Array Detector (DAD) at a wavelength of $200 \mathrm{~nm}$ to enable optimal sensitivity for nitroglycerin. Simultaneously, all peaks of the electropherogram were also scanned from 190 to $400 \mathrm{~nm}$ to confirm the identity of the compounds by comparison with the UV spectrum obtained for standards.

For mass spectral analysis, the CE system was connected to an Agilent 3250AA TOFMSD with an orthogonal spray interface. Electrospray ionization and mass spectrometer operating parameters were first optimized in direct infusion mode by injecting standards individually without any separation. Optimal parameters were found to be a source temperature of $150^{\circ} \mathrm{C}$ (to avoid capillary tip damage), $5 \mathrm{ml} / \mathrm{min}$ drying gas (nitrogen) and a 10 psig nebulizer pressure. Both positive and negative electrospray ionization modes were used to detect all compounds. Therefore, two methods were set up and run for each 
sample. In positive ion mode, optimal voltages were $4000 \mathrm{~V}$ for the capillary, $140 \mathrm{~V}$ for the fragmentor, $50 \mathrm{~V}$ for the skimmer and $300 \mathrm{~V}$ for the octopoles. In negative ion mode, optimal voltages were $5000 \mathrm{~V}$ for the capillary, $125 \mathrm{~V}$ for the fragmentor, $60 \mathrm{~V}$ for the skimmer and 300V for the octopoles. The optimized sheath liquid composition for the electrospray interface was a 75:25 v/v mixture of methanol/water containing $20 \mathrm{mM}$ ammonium nitrate. The pump was set to a flow rate of $0.5 \mathrm{ml} / \mathrm{min}$ with a $1: 100$ split ratio resulting in a net flow of $0.005 \mathrm{ml} / \mathrm{min}$. The Agilent API-TOF Reference Mass Solution Kit containing purine at m/z 121.0509 and HP0921 at m/z 922.0098 was added to the sheath flow liquid and monitored to maintain the instrument's calibration. Data analysis was performed using Applied Biosystems/MDS-SCIEX Analyst QS software (Frankfurt, Germany).

\section{Sample Preparation}

Smokeless powder standards were diluted with acetonitrile to give standard concentrations of $1.0 \mathrm{mg} / \mathrm{ml}$. Each solution was then injected in 75:25 acetonitrile: phosphate buffer solution at the desired concentration. When not in use, the stock solutions were stored in the laboratory freezer at $-18^{\circ} \mathrm{C}$.

Individual smokeless powders were prepared using a method adapted from a procedure published by Wissinger et. al. [2]. About $5 \mathrm{mg}$ of powder samples were dissolved in $250 \mu \mathrm{l}$ of methylene chloride, $200 \mu \mathrm{l}$ of supernatant were removed, dried and reconstituted in $400 \mu \mathrm{l}$ of 75:25 acetonitrile:phosphate buffer solution.

\section{F. Results and Discussion}

Coupling the CEC column to the TOF-MS allows accurate mass determination 
and identification of most of the sample components present in smokeless powders. However, the TOF-MS is not sensitive to nitroglycerin and dinitrotoluenes in the positive ion mode and requires the production of negative ion adducts so methods for both ion modes were developed in this study. The chromatographic separation was optimized using CEC-UV and the MS detection improved by direct infusion of individual standards before the CEC was directly coupled to the MS instrument.

\section{Method Development and CEC-UV Results}

The initial procedure for analysis was adapted from a previous separation developed in this laboratory for the determination of benzodiazepines [25]. Prior to interfacing the capillary to the mass spectrometer, separation parameters were examined using UVvisible detection. To maximize sensitivity of detection at $200 \mathrm{~nm}$, a phosphate buffer was used instead of the ammonium acetate buffer described in [25]. The proportion of phosphate buffer to acetonitrile contained in the eluent (or running buffer) was investigated and it was found that small variations could have large effect on the resolution of certain peaks, especially dinitrotoluene and nitrosodiphenylamine isomers. Most experiments were performed with an acetonitrile/phosphate buffer ratio of 73:27 since it usually provided the best separation for all 14 compounds. However, slight modifications of the proportions were sometimes made to adjust retention and elution time. Figure 2-9 shows the separation of the mixed standard containing 14 smokeless powder additives using this procedure. 


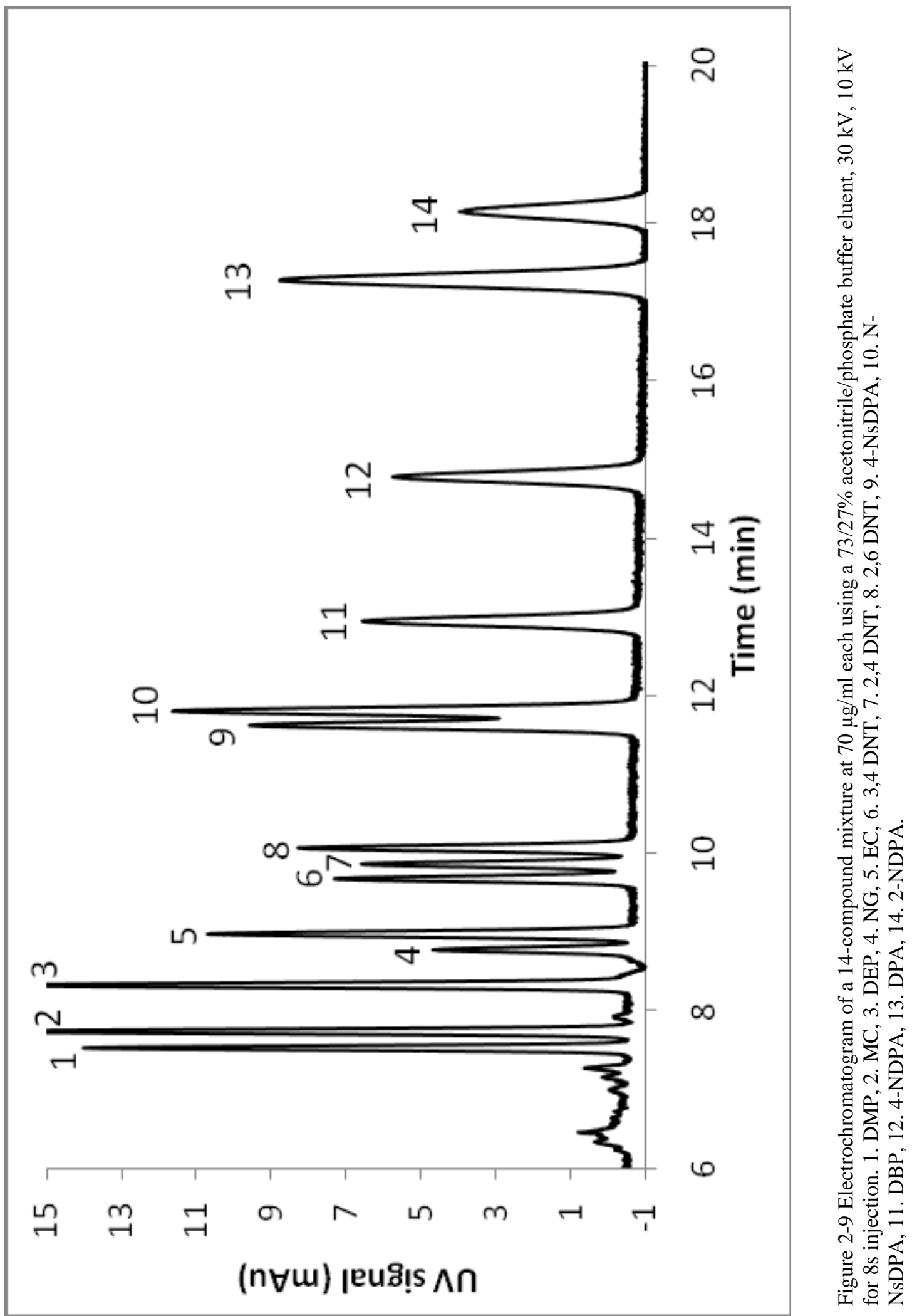


The optimized running voltage was $30 \mathrm{kV}$ on a $50 \mathrm{~cm}$ capillary. At a lower run voltage elution times (and thus analysis times) were longer and peaks were broader. No improvement was shown in any analytical parameter when run at lower voltages.

As for the solvent composition of the sample, similar results were obtained for run buffer compositions of $40 \% \mathrm{ACN} / 57.5 \%$ phosphate buffer/2.5\% MeOH and 75\% $\mathrm{ACN} / 25 \%$ phosphate buffer. When the sample was run in $100 \%$ acetonitrile both resolution and peak heights were poorer, likely because of incompatibility between the pure solvent and the buffer. Reduced solubility of the target compounds in acetonitrile may also have been a contributing factor.

Voltage and duration of the injection were also investigated. A sample containing $25 \mu \mathrm{g} / \mathrm{ml}$ of each compound was injected for 5 seconds at $5,10,15$ and $20 \mathrm{kV}$; the same concentration of sample was then injected at $10 \mathrm{kV}$ for intervals of 5, 8, 10, 15, and 20 seconds. Results showed that at higher injection voltages or longer times, peak height and width were higher but also the lower the resolution and efficiency. Also, at high voltage injection or long injection time, current instability was observed after several injections. The most stable results were obtained with a voltage lower than $15 \mathrm{kV}$ and the efficiency was shown to drop by $10 \%$ of the number of plates for an increase of $3 \mathrm{~s}$ in injection time. Therefore, the best compromise was determined to be an injection at $10 \mathrm{kV}$ for $8 \mathrm{~s}$, which provided threefold higher peak heights than at $5 \mathrm{kV}$ for $5 \mathrm{~s}$, with a loss of only $7 \%$ in resolution.

Linearity was then investigated using these optimized conditions for standards ranging from 0 to $60 \mu \mathrm{g} / \mathrm{ml}(\mathrm{n}=9)$. Repeatability was obtained by performing five sample injections at $25 \mu \mathrm{g} / \mathrm{ml}$. Results are presented in Table 2-1. 


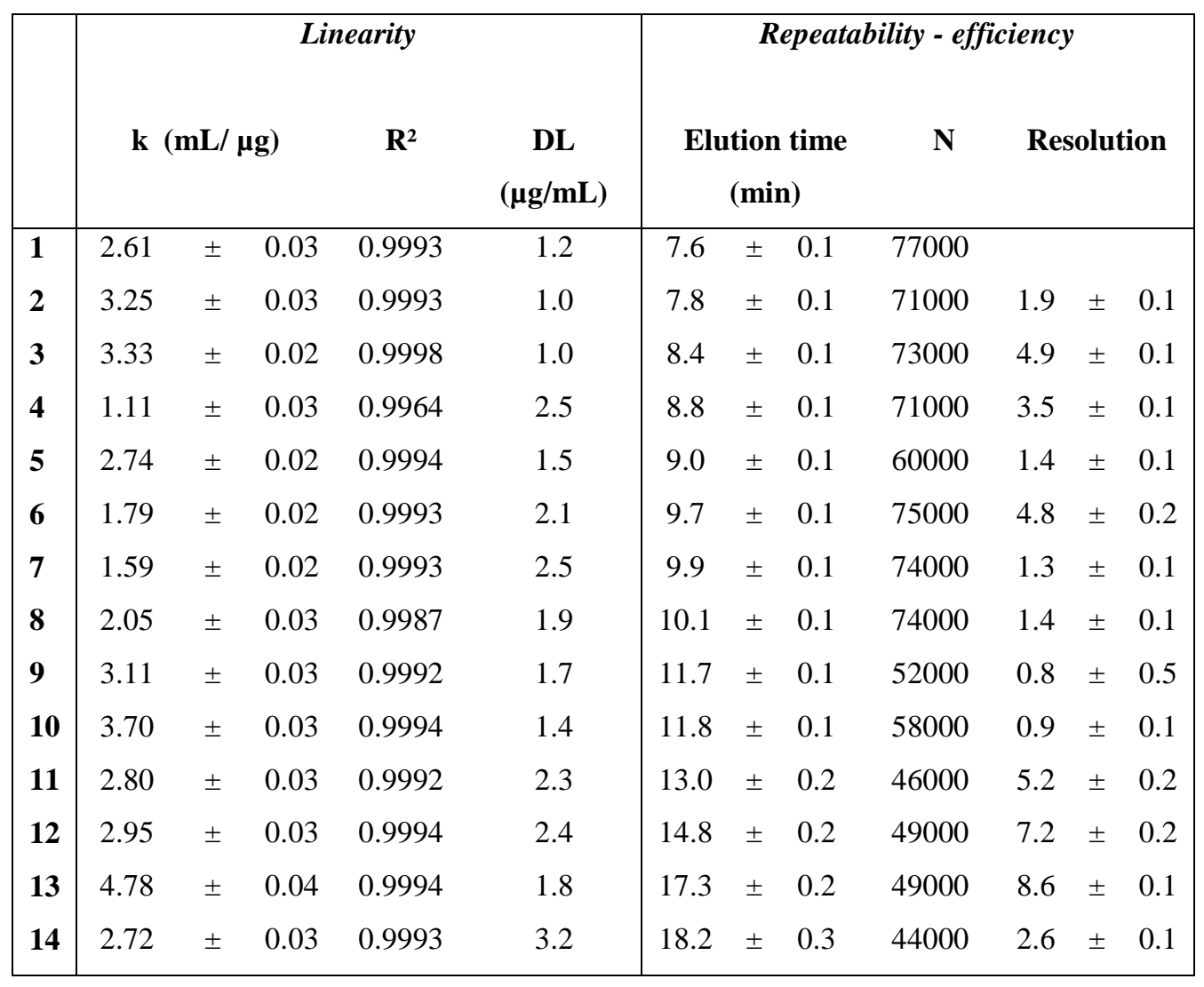

Table 2-1. Figures of merit for CEC-UV. For linearity experiments $(n=9)$ with concentrations from 0 to 60 $\mu \mathrm{g} / \mathrm{mL}$, the slope ( $\mathrm{k}$ ) was calculated as area $=\mathrm{k} \times$ concentration in $\mu \mathrm{g} / \mathrm{mL}$. DL is the detection limit, calculated from the signal obtained for the smallest concentration giving a peak and reported to a signal:noise ratio of 3 . For repeatability experiments $(n=5), 25 \mu \mathrm{g} / \mathrm{ml}$ of each compound was used. $\mathrm{N}$ is the number of theoretical plates. Conditions were as 73/27 acetonitrile/phosphate buffer eluent, $30 \mathrm{kV}, 10 \mathrm{kV}$ for 8s injection. 1. DMP, 2. MC, 3. DEP, 4. NG, 5. EC, 6. 3,4 DNT, 7. 2,4 DNT, 8. 2,6 DNT, 9. 4-NsDPA, 10. N-NsDPA, 11. DBP, 12. 4-NDPA, 13. DPA, 14. 2-NDPA.

The complete separation of the 14 compounds was performed in less than 20 minutes. 
Variability in migration times was less than $2 \%$ for every compound while variability in peak areas was $6 \pm 1 \%$. Resolution was greater than 1 for all compounds but N-nsDPA and 4-NsDPA which coelute, especially at high concentrations.

Individual smokeless powder samples were then analyzed using this technique. Figure 210 a-e presents five examples of the CEC-UV analysis of smokeless powders. The results demonstrate the high efficiency possible using the CEC method and permit easy discrimination between the various types of powders. Most samples contain nitroglycerin and diphenylamine, but every powder was shown to be different: Hodgdon 380 powder contained a high amount of dibutylphthalate, HI-SKOR 700-X had no detectable compounds detectable except for nitroglycerin and ethyl centralite, and IMR 4895 did not contain nitroglycerin (which was expected since it is a single base powder) but did contain a high amount of 2,4-dinitrotoluene. Some peaks were high enough to be significant and were thought to be 4-nitrodiphenylamine and/or 2- nitrodiphenylamine since they appeared at the appropriate elution time but their UV spectra were not intense enough to confirm their identity. Consequently it was decided that coupling CEC-UV with another, potentially more selective and/or sensitive detection method could be useful for these molecules. CEC was then coupled to MS detection in order to confirm the UV results and determine the analytical gain of this tool. 

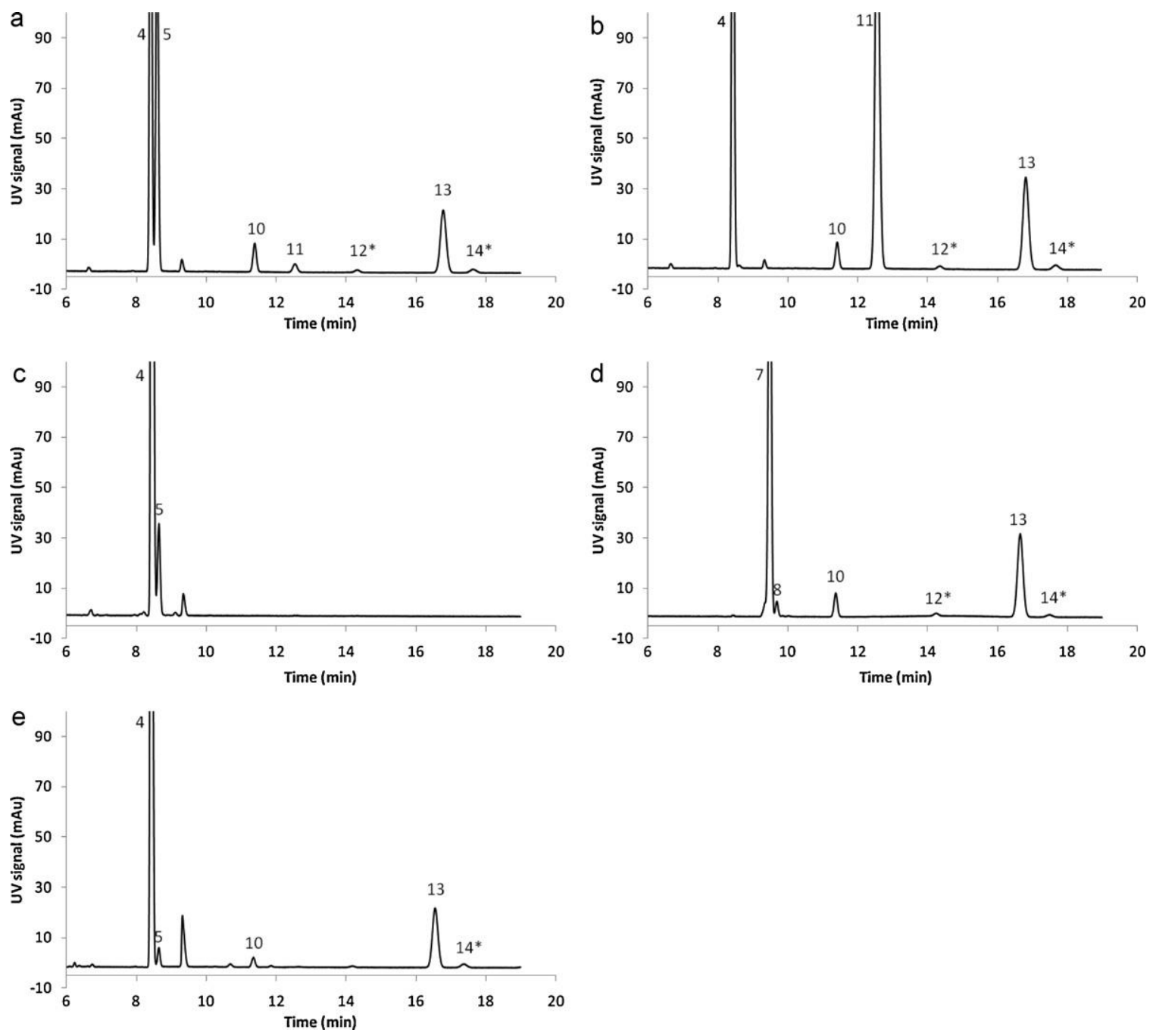

Fig. 2-10. Samples of (a) Winchester 296, (b) Hodgdon H380, (c) HI-SKOR 700-X, (d) IMR 4895, and (e) Red Dot Alliant Powder. Eluent was 79/21\% acetonitrile/phosphate buffer, $30 \mathrm{kV}$ running voltage, $10 \mathrm{kV}-$ $8 \mathrm{~s}$ injection. For better clarity, electrochromatograms have been cropped and high intensity peaks were cut off. * indicates peaks too low to permit identity to be confirmed via UV spectrum. (1) DMP, (2) MC, (3) DEP, (4) NG, (5) EC, (6) 3,4-DNT, (7) 2,4-DNT, (8) 2,6-DNT, (9) 4-NsDPA, (10) N-NsDPA, (11) DBP, (12) 4-NDPA, (13) DPA, and (14) 2-NPDA.

\section{Method Development and CEC-MS Results}

Before performing any CEC-MS experiments, MS detector parameters were optimized for smokeless powder additives. Analytes were first directly infused into the mass spectrometer using a syringe pump and scanned at different voltages in the positive electrospray ionization mode. Because of their functional groups, some of the analytes 
were not sensitive to the positive ion mode so infusion experiments were also run in the negative ion mode.

Phthalates, centralites, nitro- and nitrosodiphenylamines were detected in the positive ion mode; nitroglycerin, dinitrotoluenes, nitrosodiphenylamines and 4-nitrodiphenylamine were detected in the negative ion mode. To optimize ESI ionization, different voltages were applied to the ion optics. In the positive ion mode, capillary voltages from 3000 to $4500 \mathrm{~V}$ and fragmentor voltages from 110 to $225 \mathrm{~V}$ were investigated. Skimmer voltages varied from 40 to $110 \mathrm{~V}$. The combination that gave the highest intensities for most compounds detected in the positive ion mode was $4000 \mathrm{~V}, 140 \mathrm{~V}$ and $50 \mathrm{~V}$ for the capillary, the fragmentor and the skimmer respectively. In the negative ion mode, the capillary voltage ranged from 4000 to $5000 \mathrm{~V}$, the fragmentor voltage from 125 to $300 \mathrm{~V}$ and the skimmer voltage from 60 to $125 \mathrm{~V}$. The best voltage combination for this mode was $5000 \mathrm{~V}, 125 \mathrm{~V}$ and $80 \mathrm{~V}$ for the capillary, the fragmentor and the skimmer respectively. In general during these experiments, the intensity of individual compounds varied over the range of experiments, with some compounds more sensitive than others at a given set of parameters. In the negative ion mode, nitroglycerin was shown to be the most sensitive, followed by 3,4-DNT, 2,4-DNT and 2,6-DNT, the signal for which was about 10 times less intense than nitroglycerin. In the positive ion mode, phthalates and centralites were the most sensitive, and 4-NsDPA was the most difficult to detect since it was about 3 times less intense than the more sensitive compounds.

After the best set of conditions in both ionization modes were determined, the CEC and MS were connected and the same standards used for CEC-UV were injected in both positive and negative ion modes. With the voltages optimized previously in the infusion 
mode, all compounds gave well-resolved peaks and were easily detected, with 2,6dinitrotoluene and 4-nitrosodiphenylamine being the exceptions that were barely detected. These two compounds were more efficiently ionized at different parameters but since they are easily detected by UV, the method was considered an acceptable compromise.

The interface between the $\mathrm{CE}$ and the MS instruments is the location where the ionization of the molecules takes place, prior to being transferred into the time of flight system. The voltages applied to the electrospray are therefore what determines the sensitivity of the analysis. Another major influence on the sensitivity of the analysis is the composition of the sheath solution for the nebulizer. The first sheath liquid used was composed of 1:1 methanol and $0.1 \%$ trifluoroacetic acid (TFA) in deionized water. While an acidic solution helps protonate the sample molecules and thus improve their ionization in the positive ion mode, an acidic composition for the sheath liquid may not provide enough ionization in the negative ion mode. To improve sensitivity in the latter, $20 \mathrm{mM}$ ammonium nitrate $(\mathrm{pH}=6.8)$ was tested in place of TFA. A dramatic improvement was obtained for nitroglycerin and DNTs with the formation of nitrate adducts, and sensitivity in the positive ion mode was also increased for all compounds. Ammonium acetate was also investigated but did not provide the same level of sensitivity as ammonium nitrate. Ultimately, the sheath liquid providing the best sensitivity was 1:1 methanol and $20 \mathrm{mM}$ ammonium nitrate in deionized water. Under these conditions, major adducts detected in positive ESI mode were the protonated form of the analytes and in negative ESI mode, nitrate adducts predominated (Table 2-2). 


\begin{tabular}{|c|c|c|c|c|}
\hline & Ion mode & $\mathbf{m} / \mathbf{z}$ & Theoretical $\mathbf{m} / \mathbf{z}$ & $\begin{array}{c}\text { m/z error } \\
(\mathbf{p p m})\end{array}$ \\
\hline DMP & ESI+ & 195.0659 & {$[\mathrm{DMP}+\mathrm{H}]^{+}=195.0657$} & 1.0 \\
\hline MC & ESI+ & 241.1340 & {$[\mathrm{MC}+\mathrm{H}]^{+}=241.1341$} & -0.4 \\
\hline DEP & ESI+ & 223.0970 & {$[\mathrm{DEP}+\mathrm{H}]^{+}=223.0970$} & 0.0 \\
\hline $\mathrm{EC}$ & ESI+ & 269.1652 & {$[\mathrm{EC}+\mathrm{H}]^{+}=269.1654$} & -0.7 \\
\hline 4/N-NsDPA & ESI+ & 199.0876 & {$[\mathrm{NsDPA}+\mathrm{H}]^{+}=199.0871$} & 2.5 \\
\hline DBP & ESI+ & 279.1590 & {$[\mathrm{DBP}+\mathrm{H}]^{+}=279.1596$} & -2.1 \\
\hline 4-NDPA & ESI+ & 215.0828 & {$[\mathrm{NDPA}+\mathrm{H}]^{+}=215.0821$} & 3.3 \\
\hline DPA & ESI+ & 170.0958 & {$[\mathrm{DPA}+\mathrm{H}]^{+}=170.0970$} & -7.1 \\
\hline 2-NDPA & ESI+ & 215.0829 & {$[\mathrm{NDPA}+\mathrm{H}]^{+}=215.0821$} & 3.7 \\
\hline NG & ESI- & 288.9863 & {$\left[\mathrm{NG}+\mathrm{NO}_{3}\right]^{-}=288.9904$} & -14.2 \\
\hline $2,4 \mathrm{DNT}$ & ESI- & 244.0197 & {$\left[\mathrm{DNT}+\mathrm{NO}_{3}\right]^{-}=244.0205$} & -3.3 \\
\hline $3,4 \mathrm{DNT}$ & ESI- & 244.0208 & {$\left[\mathrm{DNT}+\mathrm{NO}_{3}\right]^{-}=244.0205$} & 1.2 \\
\hline 4/N-NsDPA & ESI- & 260.0652 & {$\left[\mathrm{NsDPA}+\mathrm{NO}_{3}\right]^{-}=260.0671$} & -7.3 \\
\hline 4-NDPA & ESI- & 276.0580 & {$\left[\mathrm{NDPA}+\mathrm{NO}_{3}\right]^{-}=276.0620$} & -14.5 \\
\hline
\end{tabular}

Table 2-2. Main adducts for smokeless powder additives standards detected in the negative and positive ion mode. The error on $\mathrm{m} / \mathrm{z}$ was calculated as the observed $\mathrm{m} / \mathrm{z}$ subtracted from the theoretical mass of the adduct and then divided by the theoretical mass.

As with CEC-UV, standards were injected from 0 to $50 \mu \mathrm{g} / \mathrm{ml}$ to determine linearity of response and 4 samples at $12 \mu \mathrm{g} / \mathrm{ml}$ were used to calculate repeatability. The results are given in Table 2-3.

Figures of merit were not determined for analytes $8-10$ (2,6-DNT, 4-NsDPA, and NNsDPA) because of the weak intensity of peaks 8 and 9 and possible coelution of peaks 9 and 10. An example chromatogram is presented in Figure 2-11 (extracted chromatograms).

The combination of good mass accuracies (Table 2-2) coupled with good repeatability of retention time provides a useful identification of the individual components in the smokeless powder samples. 
While the sensitivity was better for most compounds using CEC-MS, linearity was better using CEC-UV. Differences in detection (on-column UV vs. distal MS) also produce expected differences in migration time. The poorer linearity of response using CEC-MS may be because of the MS interface, which does not permit both capillary ends to be pressurized, as is the case with CEC-UV. For this reason, CEC-MS may be more useful as a confirmation tool in line with CEC-UV analysis, which gives more accurate quantification. The excellent repeatability of the elution times and quality of the mass spectra made the identification of compounds easy and reliable.

\begin{tabular}{|c|c|c|c|c|c|c|c|c|c|c|}
\hline & \multirow[b]{2}{*}{$\begin{array}{c}\text { ESI } \\
\text { mode }\end{array}$} & \multicolumn{5}{|c|}{ Linearity } & \multicolumn{4}{|c|}{ Repeatability - efficiency } \\
\hline & & \multicolumn{3}{|c|}{$\mathrm{k}(\mathrm{mL} / \mu \mathrm{g})$} & $\mathbf{R}^{2}$ & \multirow{2}{*}{$\begin{array}{c}\begin{array}{c}\mathrm{DL} \\
(\mu \mathrm{g} / \mathrm{mL})\end{array} \\
0.1\end{array}$} & \multicolumn{3}{|c|}{$\begin{array}{l}\text { Elution time } \\
\qquad(\min )\end{array}$} & $\mathbf{N}$ \\
\hline 1 & + & 111383 & \pm & 6755 & 0.9749 & & 7.0 & \pm & 0.1 & 9000 \\
\hline 2 & + & 399573 & \pm & 22015 & 0.9792 & 0.1 & 7.2 & \pm & 0.1 & 8700 \\
\hline 3 & + & 188835 & \pm & 21100 & 0.9196 & 0.1 & 8.0 & \pm & 0.1 & 12000 \\
\hline 4 & - & 95248 & \pm & 7716 & 0.9442 & 0.2 & 8.2 & \pm & 0.1 & 8200 \\
\hline 5 & + & 423130 & \pm & 36826 & 0.9496 & 0.1 & 8.7 & \pm & 0.1 & 4900 \\
\hline 6 & - & 3587 & \pm & 402 & 0.8983 & 2.9 & 9.3 & \pm & 0.1 & 24000 \\
\hline 7 & - & 1560 & \pm & 111 & 0.9567 & 5.5 & 9.5 & \pm & 0.1 & 19000 \\
\hline 8 & - & \multicolumn{5}{|c|}{ Not detected } & \multicolumn{4}{|c|}{ Not detected } \\
\hline 9 & + & \multicolumn{5}{|c|}{ Not detected } & \multicolumn{4}{|c|}{ Not detected } \\
\hline 10 & + & \multicolumn{5}{|c|}{ Not detected } & \multicolumn{4}{|c|}{ Not detected } \\
\hline 11 & + & 188633 & \pm & 7919 & 0.9878 & 0.1 & 13.6 & \pm & 0.1 & 15000 \\
\hline 12 & + & 142085 & \pm & 5214 & 0.9907 & 0.2 & 16.7 & \pm & 0.2 & 15000 \\
\hline 13 & + & 50069 & \pm & 6762 & 0.8868 & 0.6 & 20.0 & \pm & 0.2 & 57000 \\
\hline 14 & + & 60710 & \pm & 6296 & 0.9300 & 0.9 & 21.0 & \pm & 0.2 & 23000 \\
\hline
\end{tabular}

Table 2-3. Figures of merit for CEC-MS. For linearity experiments ( $n=9)$, concentrations from 0 to 60 $\mu \mathrm{g} / \mathrm{mL}$, the slope ( $\mathrm{k}$ ) was calculated as area $=\mathrm{k} \times$ concentration in $\mu \mathrm{g} / \mathrm{mL}$. DL is the detection limit, calculated from the signal obtained for the smallest concentration giving a peak and reported to a signal: noise ratio of 3 . For repeatability experiments $(\mathrm{n}=4), 24 \mu \mathrm{g} / \mathrm{ml}$ of each compound was used. $\mathrm{N}$ is the number of theoretical plates. Conditions were as 73/27 acetonitrile/phosphate buffer eluent, $30 \mathrm{kV}, 10 \mathrm{kV}$ for 8s injection. 1. DMP, 2. MC, 3. DEP, 4. NG, 5. EC, 6. 3,4 DNT, 7. 2,4 DNT, 8. 2,6 DNT, 9/10. 4NsDPA/N-NsDPA, 11. DBP, 12. 4-NDPA, 13. DPA, 14. 2-NDPA. 


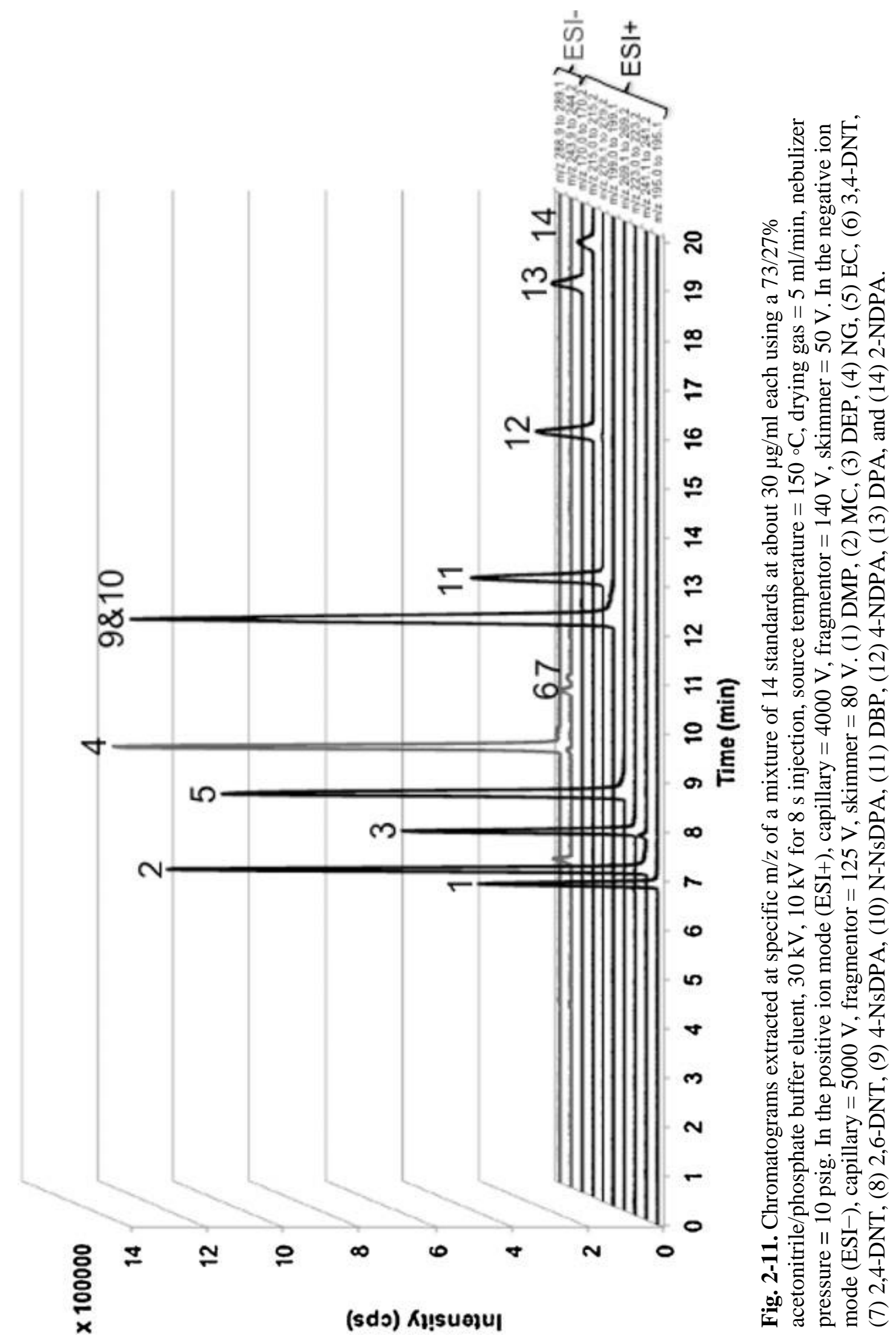


When comparing MS and UV results of smokeless powder samples, both methods can be complementary to each other. UV, contrary to MS, allows the detection of 2,6DNT and the separation of N-NsDPA and 4-NsDPA. However, MS allows confirmation of the identity of 2-NDPA and 4-NDPA when they are present in low amounts. MS, since it is more sensitive than UV, allows the detection of compounds that could not be detected by UV because of their low concentrations: e.g. MC in Winchester 296 and HI-SKOR 700-X, DEP in HI-SKOR 700-X, and 4-NDPA in HI-SKOR 700-X and Alliant Red Dot Powder. Because each mass gives a different chromatogram, a small amount of EC was detected by MS which certainly coeluted in the high nitroglycerin peak in the UV chromatogram of Hodgdon H380.

\section{G. Conclusion}

This report presents an assessment of the first use of CEC-UV and CEC-MS methods for the analysis of compounds present in smokeless powders. The procedure uses in-situ polymerization of acrylate monoliths for separation. The method produces high resolution separations by CEC-UV and exact mass determination of many of the individual components present in these powders by time-of-flight mass spectrometry. The procedure should prove useful for the determination of the composition of unburned powders used in improvised explosive devices as well as for comparison of preparation and brands of these powders. The relatively small injection sizes permitted by CEC will permit ready analysis of submissions where minimal sample is available, making this a useful procedure in forensic analysis. 


\section{References}

[1] Stine, G.Y., Anal. Chem. 63 (1991) 475A.

[2] Wissinger, C.E.; McCord, B.R., J. Forensic Sci. 47 (2002) 168.

[3] Heramb, R.B., McCord, B.R., Forensic Sci. Commun. 4 (2002).

[4] Cascio, O., Trettene, M., Bortolotti, F., Milana, G., Tagliaro F., Electrophoresis 25 (2004) 1543.

[5] Joshi, M., Rigsby, K., Almirall, J.R., Forensic Sci. Int. 208 (2011) 29.

[6] Scherperel, G., Reid, G., Smith, R.W., Anal. Bioanal. Chem. 394 (2009) 2019.

[7] Tong, Y., Wu, Z., Yang, C., Yu, J., Zhang, X., Yang, S., Deng, X., Xu, Y., Wen, Y., Analyst 126 (2001) 480.

[8] Mathis, J.A., McCord, B.R., J. Chromatogr. A 988 (2003) 107.

[9] Meng, H.-H., Caddy, B., J. Forensic Sci. 42 (1997) 553.

[10] Goudsmits, E., Sharples, G., Birkett, J.W., TrAC, Trends Anal. Chem. 74 (2015) 46.

[11] Stankovic, M., Vujovic, B., Filipovi, M., Chromatographia 42 (1996) 593.

[12] Zhao, X., Yinon, J., J. Chromatogr. A 977 (2002) 59.

[13] Hopper, K., McCord, B., J. Forensic Sci. 50 (2005) 307-315.

[14] Hopper, K.G., LeClair, H., McCord, B.R., Talanta 67 (2005) 304.

[15] Northrop, D.M., Martire, D.E., MacCrehan, W.A., Anal. Chem. 63 (1991) 1038.

[16] Smith, K.D., McCord, B.R., MacCrehan, W.A., Mount, K., Rowe, W.F., J. Forensic Sci. 44 (1999) 789.

[17] Northrop, D.M., J. Forensic Sci. 46 (2001) 549.

[18] Silva, M., Electrophoresis 28 (2007) 174.

[19] Yang, L., Lee, C.S., J. Chromatogr. A 780 (1997) 207. 
[20] Krull, I.S., Stevenson, R.L., Mistry, K., Swartz, M.E., Capillary Electrochromatography and Pressurized Flow Capillary Electrochromatography, HNB Publishing, New York, NY, 2000.

[21] Bailey, C.G., Yan, C., Anal. Chem. 70 (1998) 3275.

[22] Giordano, B.C., Terray, A., Collins, G.E., Electrophoresis 27 (2006) 4295.

[23] Quirino, J., Haddad, P.R., Electrophoresis 30 (2009) 1670.

[24] Zhang, Z-X., Yan, B., Liu, K., Liao, Y.P., Liu, H.W., Electrophoresis 30 (2009) 379.

[25] Blas, M., McCord, B.R., Electrophoresis 29 (2008) 2182.

[26] Barz, D., Ehrhard, P., Lab Chip 5 (2009) 949.

[27] Rathore, A.S., J. Chromatogr. A 1037 (2004) 431.

[28] Knox, J.H., Grant, I.H., Chromatographia 32 (1991) 317.

[29] Dole, M., Mack, L.L., Hines, R.L., Mobley, R.C., Ferguson, L.D., Alice, M.B.J., J. Chem. Phys. 49 (1968) 2240.

[30] Iribarne, J.V., Thomson, B.A., J. Chem. Phys. 64 (1976) 2287.

[31] Cole, R.B., Electrospray and MALDI Mass Spectrometry, $2^{\text {nd }}$ ed.; John Wiley and Sons: Hoboken, NJ, 2010; p. 19.

[32] de Hoffman, E., Stroobant, V., Mass Spectrometry: Principles and Applications, $3^{\text {rd }}$ ed.; John Wiley and Sons: Chichester, Sussex, UK, 2007; p. 91

[33] Ngola, S.M., Fintschenko, Y., Choi, W., Shepodd, T.J., Anal. Chem. 73 (2001) 849.

[34] Delaunay-Bertoncini, N., Demesmay, C., Rocca, J., Electrophoresis 25 (2004) 3204.

[35] Agilent Technologies, Inc., Agilent G3250AA LC/MSD TOF System Concepts Guide, $5^{\text {th }}$ Ed., 2007; Agilent, Santa Clara, CA. 


\section{CHAPTER 3: Detection of the Improvised Explosives Ammonium Nitrate and Urea Nitrate using Nonaqueous Solvents with Electrospray Ionization and MS/MS Detection}

\section{Introduction}

Ammonium nitrate and urea nitrate are salts of nitric acid that are used in improvised explosive devices (IEDs). Ammonium nitrate (AN) is a common fertilizer and was first used as a blasting agent in 1867 . Over the years, AN has become the most widely used mining explosive and is typically mixed with fuel oil prior to application [1]. It is one of the most common industrial chemicals, with a global production of 485,000 tons per year in 2005 .

Ammonium nitrate is also routinely used in improvised explosive devices and has garnered much attention for its use in the Middle East. In the United States, it was most notably used in 1995 to destroy the Alfred P. Murrah Federal Building in Oklahoma City, Oklahoma [2].

Urea nitrate (UN) was mostly unknown to the general public until it was used in the first World Trade Center bombing in 1993. This explosive has become an alternative to ammonium nitrate (AN) in improvised devices. Both urea nitrate and ammonium nitrate are easily obtained or synthesized from largely unregulated materials and have been employed in bombing incidents around the world. AN (with PETN) was also used in the Delhi bombing in 2011 [3], and UN has been used by Palestinian separatists against the Israeli military [4]. The import of urea fertilizer was banned by the Peruvian government in 1992 because UN was a favorite explosive of the Shining Path guerrilla organization [5], and the governments of Pakistan and Afghanistan have banned the use of AN 
because of its diversion from farming to production of ANFO [6]. UN is also a popular topic of discussion on "citizen scientist" $[7,8]$ websites because of its easily - sourced ingredients and ease of manufacture.

\section{Ammonium Nitrate}

Ammonium nitrate (AN) is most commonly used to supply nitrogen to agricultural crops and is also used as a blasting agent used in mining operations. It can be mixed with a wide variety of metallic or organic fuels to produce blasting agents. These mixtures are stable and require a booster and a commercial or homemade blasting cap to be detonated. The velocity of detonation of ammonium nitrate is approximately 17, 290 feet/second. AN occurs naturally as a mineral and was historically mined from deposits in South America, but now almost all ammonium nitrate is produced on an industrial scale by combining nitric acid and gaseous ammonia to produce solid ammonium nitrate. AN is generally supplied as small white spherical pellets called prills. The prills may be either fertilizer grade or blasting grade. Blasting grade AN prills are more porous than fertilizer - grade AN, making it easier for the prills to absorb the fuel oil that is used in commercial AN blasting, increasing the strength and reactivity of the mixture [9]. Other factors that increase the strength of the blast include confinement of the mixture, and the amount and type of fuel used.

\section{Urea Nitrate}

Urea nitrate (UN) is an improvised explosive made from urea fertilizer and nitric acid. The first bombing of the World Trade Center in 1993 was the first time most Americans 
had ever heard of UN. Its destructive power is similar to AN, with a detonation velocity of approximately 15, 420 feet/second. UN is made by combining urea with nitric acid. Even though it is stable, it is not used for commercial or military purposes because it absorbs water from the atmosphere upon standing, limiting its effectiveness and making it so corrosively acidic that it cannot be stored in metal containers or used to fill projectiles.

\section{B. Analytical Techniques}

\section{Ammonium Nitrate}

AN may be identified by positive ionization mode electrospray ionization - mass spectrometry (ESI - MS) [10], ion chromatography [11, 12], capillary electrophoresis [13], ion mobility spectrometry [14], and Fourier transform infrared spectroscopy [15]. Ammonium and nitrate ions may be detected by ion chromatography (IC) [16] or color test reagents such as the diphenylamine test for nitrate ion or the Nessler reagent for ammonium ion, but no color test for the $\mathrm{AN}$ ion pair exists.

Residual amounts of ammonium nitrate [10] and urea nitrate [24] can be quickly and selectively determined by electrospray ionization - mass spectrometry (ESI - MS). Ammonium nitrate is detected in positive electrospray mode and urea nitrate in negative electrospray mode.

A technique for the detection of both $\mathrm{AN}$ and $\mathrm{UN}$ in positive ESI mode using infusion with crown ethers exists [25]. Photodissociation followed by laser-induced fluorescence has also been used to detect both compounds [26]. 


\section{Urea Nitrate}

UN may be presumptively identified using para - dimethylamino cinnamaldehyde ( $p-$ DMAC) [17]. UN may also be detected using $X$ - ray diffraction [18], infrared spectroscopy [19], voltammetry [20], and positive ionization mode ESI - MS when complexed with crown ethers [21], and by LC - UV/fluorescence using xanthydrol as a fluorophore [22]. Almog expanded on the use of xanthydrol for the detection of UN by a further reaction with alcohol to form a compound amenable to GC/MS analysis [23].

\section{Goals of This Project}

The current work was built upon the previous ESI - MS work performed by Tamiri and coworkers to demonstrate the effects of common interferences on the detection of AN and $\mathrm{UN}$, and also to address the observation that under certain conditions, a signal that can be mistaken for urea nitrate is generated as an artifact of ionization conditions in samples that contain a mixture of urea and a nitrate source [21, 24]. While the improvised explosive nature of urea nitrate is of importance to the military and to law enforcement, urea is a legal chemical that by itself has no explosive tendencies. Although it is the precursor to the explosive, an incorrect analytical result taken out of context could sidetrack an important investigation and/or send an innocent person to prison. The ability to differentiate between the use of these compounds as fertilizers vs explosives is an important goal of any analytical procedure.

Following the manufacture or use of an IED, AN and UN may be present as residue on the hands or clothing of individuals, or on surfaces they may have touched. Detection is 
performed on samples collected by swabbing or extracting cuttings from the surfaces. However, an individual suspected of handling AN or UN may have other potentially interfering substances such as soil, food products, grease, or oil on their skin or clothes.

Any technique used for rapid identification must be able to detect AN and UN in the presence of such interferences. Unfortunately both these salts undergo a metathetical reaction in polar protic solvents such as water or methanol and can freely combine with other salts. Furthermore, urea nitrate decomposes to neutral urea and nitric acid in water. Because urea, nitrate and ammonium ions are present in the environment [29] and because urea is a ubiquitous ingredient in consumer health and beauty products, their presence in the sample in any combination cannot be taken to mean that an explosive was originally present, particularly in situations where the explosive was present in trace quantities. In order to positively determine the presence of these salts, a technique such as FTIR is typically used; however, this procedure lacks sensitivity and specificity. New techniques are needed that are capable of specific detection of trace amounts of these explosives. It is the goal of this thesis to develop such a procedure using nonaqueous methods capable of detecting trace levels of these salts in the form of ion pairs.

In this project, a procedure for the rapid detection of ammonium nitrate and urea nitrate was developed with a goal to permit the differentiation of urea nitrate from a mixture of urea with nitrate salts. The procedure involves the use of direct infusion into a triplequadrupole mass spectrometer with an electrospray ionization source (ESI-QqQ-MS). The specificity of the procedure was examined by testing the method against potential interferences that can be seen in environments where samples are commonly taken. 
The procedure increases sample stability and does not require the use of crown ethers. Furthermore, the method can be used to differentiate urea nitrate from the mass spectral signal generated by mixtures of urea and nitrate salts through the use of collision induced dissociation in nonaqueous solvents.

\section{Sample Preparation}

\section{Chemicals and Reagents}

Urea nitrate was synthesized in-house and its structure confirmed by FTIR. Ammonium nitrate, potassium nitrate, and 2-methoxyethanol (methyl cellosolve) were purchased from Sigma-Aldrich. Urea standards were purchased from Beckman Coulter. Formic acid and methanol (both Optima LC/MS grade) were purchased from Fisher. Acetone (reagent grade) was purchased from Pharmco-AAPER. Para-dimethylamino cinnamaldehyde ( $p$-DMAC) was purchased from Acros Organics. Standard solutions were prepared in $95 \%$ acetone/5\% 2-methoxyethanol.

\section{a. Choice of Dissolution Solvent for Urea Nitrate}

In aqueous and polar protic solvents, ammonium nitrate dissociates into its constituent anion and cation; however, the UN molecule undergoes a decomposition reaction to urea and nitric acid. Because these ions are so common in the environment, the presence of nitrate ion with neutral urea or the ammonium ion in a sample cannot be taken to mean that ammonium nitrate or urea nitrate was present originally. To prove the presence of these salts, it is necessary to capture the intact ion pair during the analysis. 
The sample must be solubilized in a non-aqueous solvent and directly sprayed into the mass spectrometer. In order to make this happen, it was first necessary to find a solvent in which UN was soluble but would not dissociate. Approximately $2 \mathrm{mg}$ of UN was added to $1 \mathrm{~mL}$ of the chosen solvent and dissolution of the solid UN was observed. Next, $50 \mu \mathrm{L}$ of $p$-DMAC color test reagent was added. The development of a red color confirmed that the uronium (protonated urea) ion was present and the solvent did not dissociate the UN pair. The results of the UN solvent tests are summarized in Table 1. Some solvents gave an initial positive result that faded as the uronium ion was dissociated by the solvent.

\section{b. Mechanism of $p$-DMAC Color Formation}

$p$-DMAC is a sensitive and specific color test for the detection of urea nitrate. It reacts with the uronium (protonated urea) ion to form a colored complex that has the structure of a protonated Schiff base as one of its resonance structures (Figure 3-1).

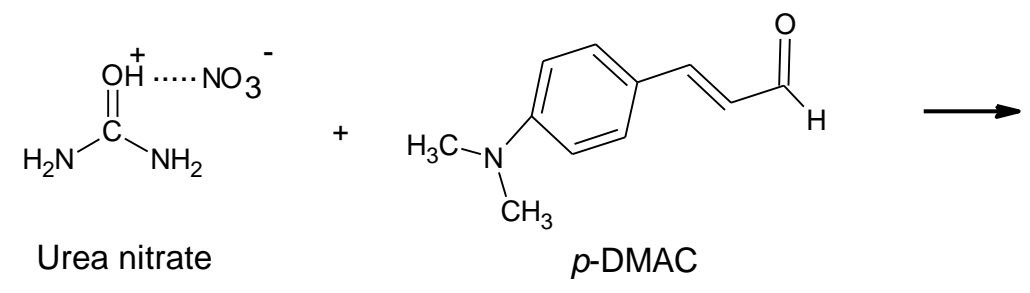<smiles>CN(C)c1ccc(/C=C/C=[N+](\ON(O)O)C(N)=O)cc1</smiles>

Figure 3-1. Resonance structures of the colored complex formed with urea nitrate and $p$-DMAC. 


\section{c. Choice of Dissolution Solvent for Ammonium Nitrate}

The solvents that did not dissociate the UN ion pair were further evaluated for their ability to dissolve AN.

AN was added by $1 \mathrm{mg}$ amounts to $1 \mathrm{~mL}$ of the solvents of interest and visually checked for dissolution. The results for AN are shown in Table 2. The solvent chosen after evaluation was a mixture of 95\% acetone with 5\% 2-methoxyethanol (methyl cellosolve, ethylene glycol monomethyl ether), which enhanced the dissolution of AN [27].

\begin{tabular}{ll}
\hline Solvent & Result \\
\hline n-Propanol & Positive \\
\hline Isopropyl alcohol (IPA) & Strong positive, fades \\
Dimethylformamide & Strong positive \\
Ethyl acetate & Positive \\
n-Butyl chloride & UN did not dissolve \\
Acetonitrile & Positive \\
Deionized water & Negative \\
Methylene chloride & Positive \\
Acetone & Positive \\
1-Decanol & UN did not dissolve \\
Ethanol & Positive, fades \\
95\% Acetone/5\% 2-methoxyethanol & Positive \\
Methanol & Positive, fades quickly \\
\hline
\end{tabular}

Table 3-1. Response of UN on addition to different solvents. A positive result indicates the development of a red color with $p$-DMAC reagent.

\begin{tabular}{ll}
\hline Solvent & Result \\
\hline n-Propanol & Insoluble \\
\hline Isopropyl alcohol (IPA) & Insoluble \\
\hline Dimethylformamide & Soluble \\
Ethyl acetate & Insoluble \\
\hline Acetonitrile & Insoluble \\
\hline Methylene chloride & Insoluble \\
Acetone & Soluble \\
Ethanol & Soluble \\
95\% Acetone/5\% 2-methoxyethanol & Soluble \\
\hline
\end{tabular}

Table 3-2. Response of AN to dissolution in different solvents. 
While both compounds show the greatest solubility in dimethylformamide (DMF), the solvent was rejected because of its potential for adverse health effects, particularly in women [28]. While it is not a carcinogen, it is classed as a reproductive toxin.

\section{Method Development}

\section{a. Instrumentation}

A Quattro Micro triple-quadrupole mass spectrometer (Waters Corporation, Milford, MA) with ESI interface was used. Samples were introduced using a $250 \mu \mathrm{L}$ syringe (Hamilton Co., Reno, Nevada) at a flow rate of $75 \mu \mathrm{L}$ per minute. A mobile phase and ionization promoter of $0.05 \%$ concentrated formic acid in methanol was infused at $25 \mu \mathrm{L}$ per minute. Since ammonium nitrate was one of the compounds of interest in this project it was decided not to use an ammonium ion - containing modifier such as ammonium acetate in favor of formic acid. This avoided having to determine the effects of an ammonium based modifier on the analytical results.

Sample and mobile phase were mixed at a T-fitting and introduced directly into the spray chamber. The optimal conditions in both positive and negative ionization modes were determined experimentally by adjusting capillary and cone voltages, and the source and desolvation temperatures. The temperature and flow rates of the desolvation gas and cone gas were also optimized.

\section{b. Sample Ionization Parameters}

The optimum ionization conditions for ammonium nitrate, urea nitrate, and urea were experimentally determined in the following conditions give the best abundance. In 
negative ESI mode, the conditions were as follows: capillary voltage, $4.0 \mathrm{kV}$; cone voltage $-40 \mathrm{~V}$, source temperature $100^{\circ} \mathrm{C}$ and desolvation temperature $100^{\circ} \mathrm{C}$. The nitrogen desolvation gas flow was 100 L/hour and the cone gas flow was turned off. For positive ESI all conditions were the same except for the cone voltage, which was set at $+40 \mathrm{~V}$. For MS/MS analysis, all conditions were the same with the addition of argon is the collision gas.

\section{MS/MS Analysis and Detection Limits}

\section{a. Ammonium Nitrate}

Ammonium nitrate and synthesized urea nitrate were dissolved in 95\% acetone/5\% 2 methoxy ethanol and run singly in positive and negative ESI modes. Best results for ammonium nitrate were obtained in the positive ion mode, and for urea nitrate, the negative ion mode. Figure 3-2 is the mass spectrum of AN obtained in $\mathrm{ESI}^{+}$mode. Two adducts of $\mathrm{AN}$ are present in the spectrum: $\mathrm{m} / \mathrm{z} 178[2 \mathrm{AN}+\mathrm{NH} 4]^{+}$and $\mathrm{m} / \mathrm{z} 258[3 \mathrm{AN}+$ $\left.\mathrm{NH}_{4}\right]^{+}$. For AN, the $\mathrm{m} / \mathrm{z} 258$ ion was chosen for quantitation. While the linearity of the calibration curve associated with each ion was similar, the uncertainty value for the data points on the $\mathrm{m} / \mathrm{z} 258$ curve was lower overall. The detection limit for $\mathrm{AN}$ was $0.3 \pm 0.09$ $\mathrm{mM}$ with a quantitation limit of three $\pm 0.9 \mathrm{mM}(30 \mathrm{ppm})$. The linear range of the calibration curve was from $60-400 \mathrm{ppm}$ with an $\mathrm{R}^{2}$ value of 0.972 . 


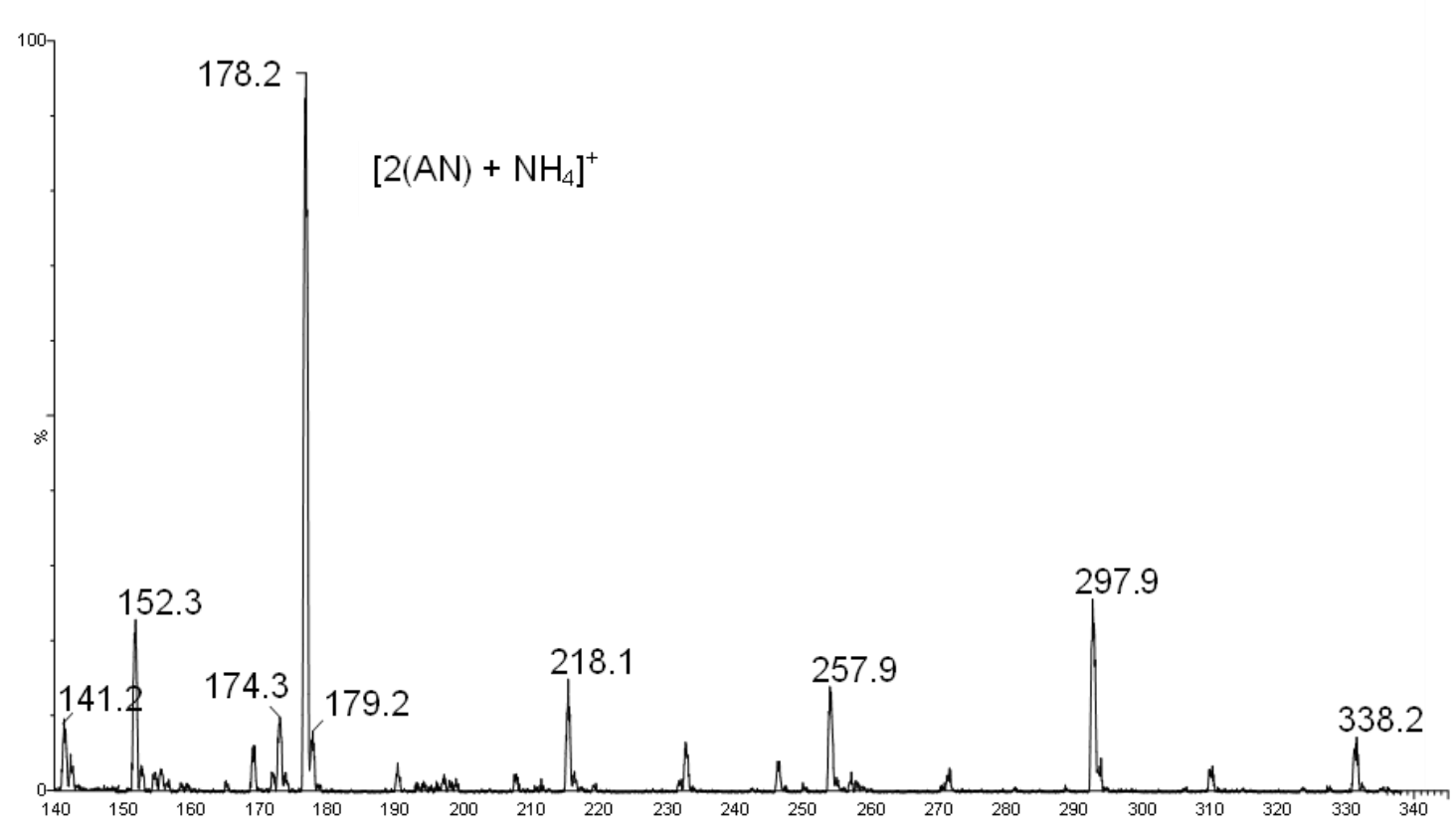

Figure 3-2. Positive ESI mass spectrum of $10 \mathrm{mM}$ ammonium nitrate (AN) standard.

\section{b. Urea Nitrate}

The spectrum of UN obtained in negative ESI mode also contained two adducts, m/z 185 $\left[\mathrm{UN}+\mathrm{HNO}_{3}\right]^{-}$and $\mathrm{m} / \mathrm{z} 248\left[\mathrm{UN}+\mathrm{HNO}_{3}+\mathrm{NO}_{3}\right]^{-}$(Figure 3-3). Quantitation was performed using the m/z 185 and m/z 248 ions. Detection limits for AN and UN were determined using the infusion procedure described above. For UN, m/z 248 was determined to be the better ion for quantitation. The detection limit for the $\mathrm{m} / \mathrm{z} 248$ ion was $0.4 \pm 0.13 \mathrm{mM}$ with a quantitation limit of $4 \pm 1.4 \mathrm{mM}(50 \mathrm{ppm})$. The linear range of the calibration curve was from $75-600 \mathrm{ppm}$ with an $\mathrm{R}^{2}$ value of 0.930 . 


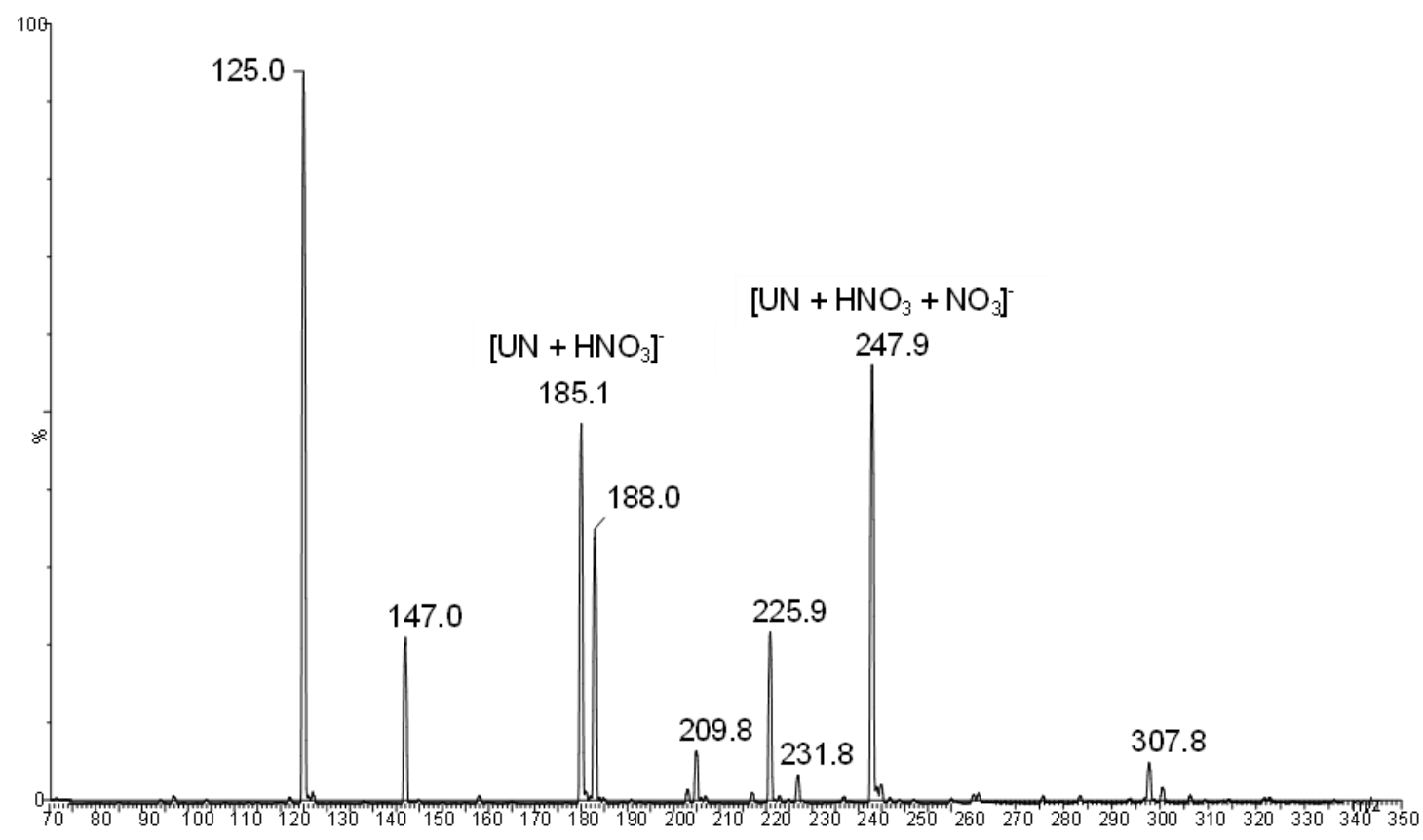

Figure 3-3. Negative ESI mass spectrum of $10 \mathrm{mM}$ urea nitrate (UN) standard.

\section{Differentiation of Urea Nitrate from Mixtures of Urea and Nitrate Salts}

Tamiri $[15,18]$ noted the presence of $\mathrm{m} / \mathrm{z} 185$ and $\mathrm{m} / \mathrm{z} 248$ ions in solutions of urea and ammonium nitrate in methanol under the conditions of the electrospray process and suggested that the mass spectral data had the potential to offer a way to determine the presence of true UN from the spurious formation of the $\mathrm{m} / \mathrm{z} 185$ signal in samples containing mixtures of urea, nitrate ion, and a proton source. As a result, the potential for formation of AN and $\mathrm{UN}$ from certain mixtures of ionic salts under electrospray conditions was examined. While this would remain a possibility for a mixture of nitrate and ammonium salts, we believed that higher specificity would be possible in the determination of urea nitrate due to the necessity of the transfer of a proton to the urea molecule during its formation. For this experiment, negative electrospray ionization coupled to a triple quadrupole mass spectrometer was used in combination with collision 
induced dissociation to simultaneously determine mixtures of urea and ammonium nitrate. The concentration of one compound was held constant $10 \mathrm{mM}$ while the other was added at $1 \mathrm{mM}, 5 \mathrm{mM}$, and $10 \mathrm{mM}$ amounts. In the first experiment, the urea concentration in the samples was held constant at $10 \mathrm{mM}$. Ammonium nitrate was added in concentrations of $1 \mathrm{mM}, 5 \mathrm{mM}$, and $10 \mathrm{mM}$ (Figure 3-4). For all mixtures a peak of varying size at m/z 185 was present, showing the formation of the $\left[\mathrm{UN}+\mathrm{NO}_{3}\right]^{-}$adduct under electrospray conditions. False UN spectra, however, can be distinguished from true urea nitrate by the presence of a peak at $\mathrm{m} / \mathrm{z} 182$ which is not present in the spectrum of urea nitrate. As the concentration of ammonium nitrate increases relative to the urea concentration, the abundance of the m/z 182 peak decreases (Figure 3-5). Additionally, there are height differences in the m/z 248 peak in relation to the $\mathrm{m} / \mathrm{z} 185$ peak.

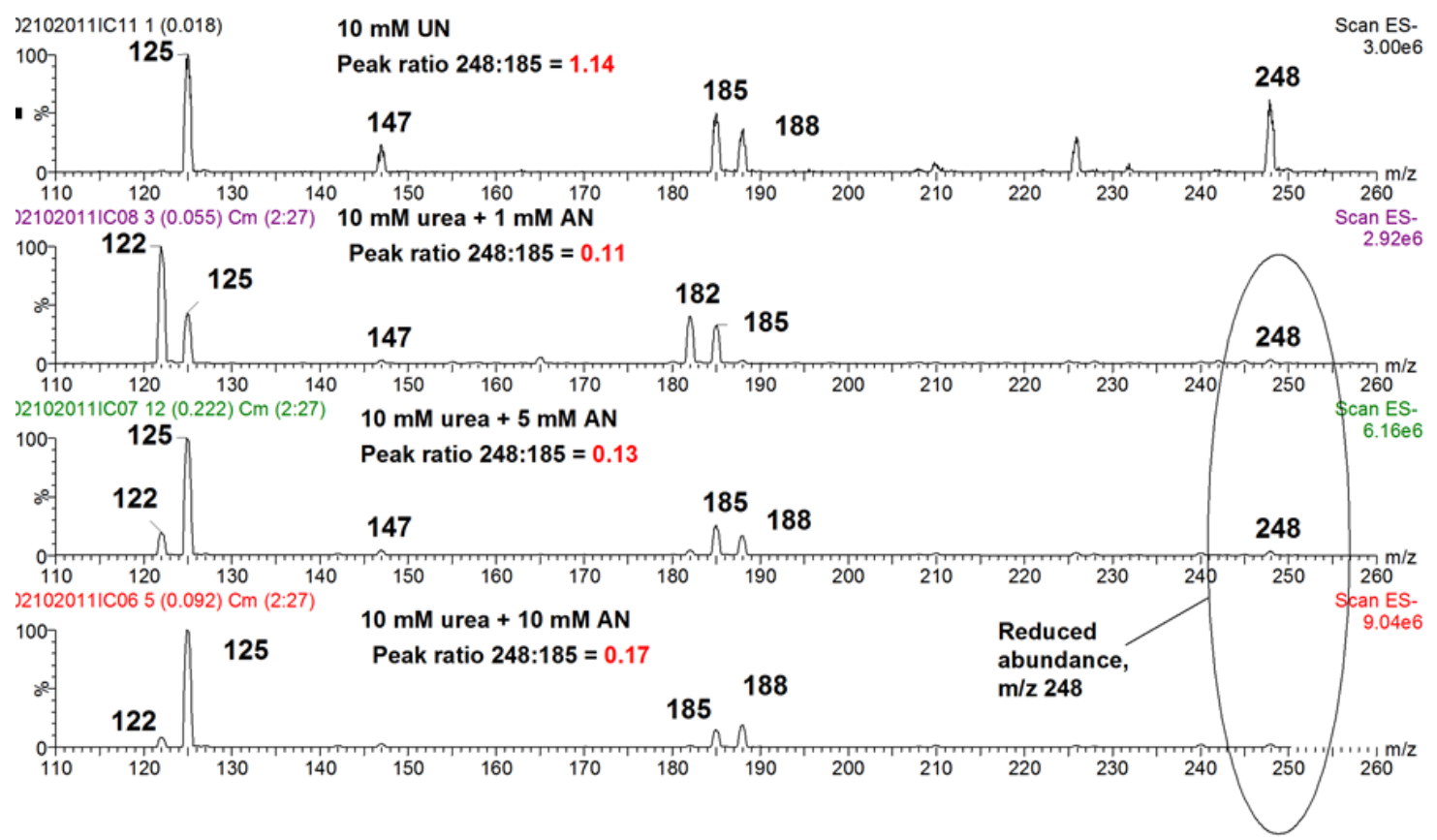

Fig. 3-4. Comparison of negative ESI mass spectrum of $10 \mathrm{mM} \mathrm{UN} \mathrm{(top)} \mathrm{with} \mathrm{negative} \mathrm{ESI} \mathrm{mass} \mathrm{spectra} \mathrm{of}$ $10 \mathrm{mM}$ urea with varying amounts of AN. Note the presence of the $\mathrm{m} / \mathrm{z} 182$ peak in the mixtures; this peak is not present in a true UN sample. Also note that in true UN the ratio of the 248:185 peaks is greater than 1.0. In the mixtures, the $248: 185$ ratio is less than 0.2 . 
All samples syringe infusion, $\mathrm{ESI}-$, mobile phase $=0.05 \% \mathrm{FA}$ in $\mathrm{MeOH}$

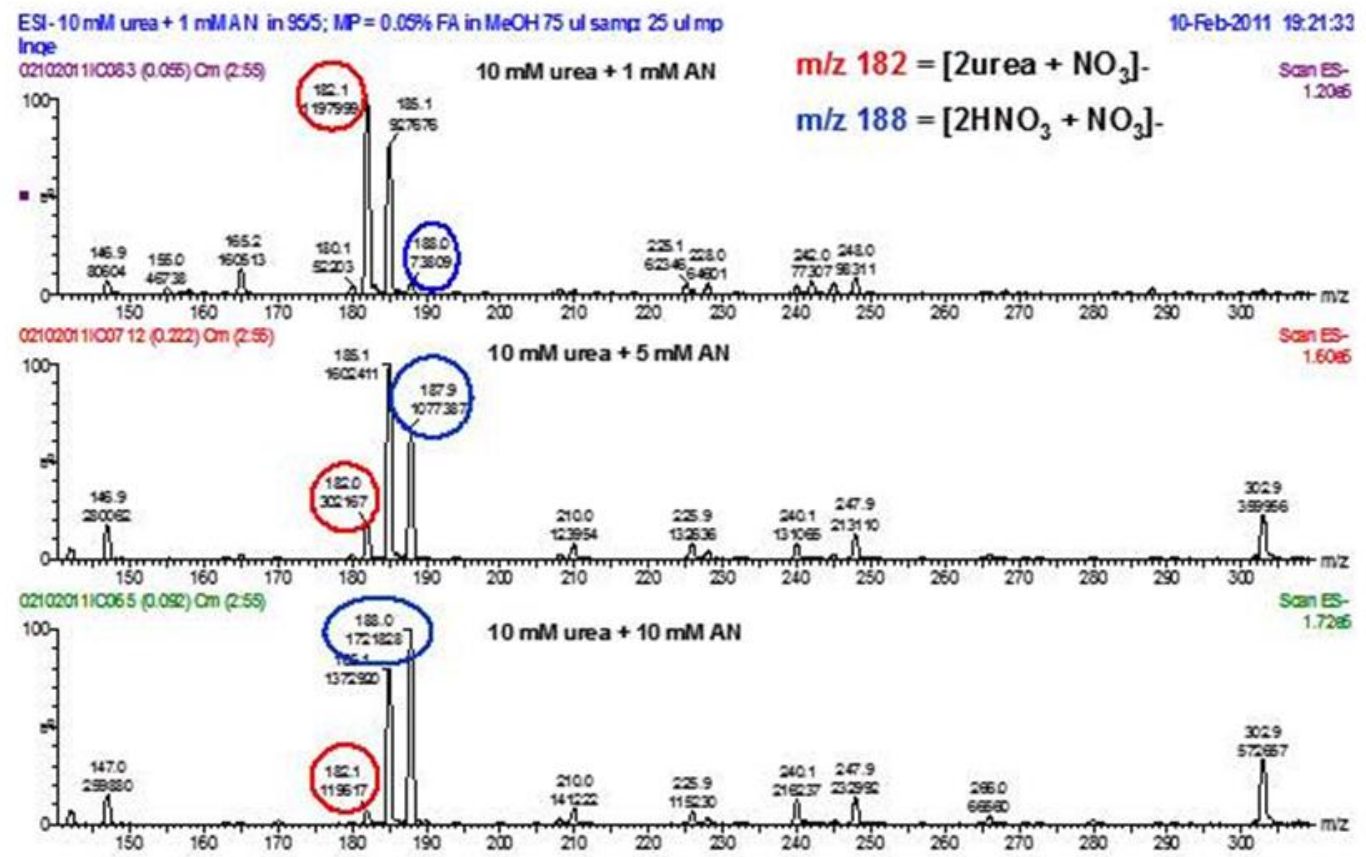

Figure 3-5. Closeup of negative ESI spectrum of urea and AN mixtures with urea held constant at $10 \mathrm{mM}$.

Following these, standards with $10 \mathrm{mM}$ ammonium nitrate and concentrations of urea at 1,5 , and $10 \mathrm{mM}$ were run and compared to the urea nitrate standard. The abundance of the $\mathrm{m} / \mathrm{z} 185$ peak increases with increasing urea concentration and again the ratio of $\mathrm{m} / \mathrm{z}$ 248: $\mathrm{m} / \mathrm{z} 185$ is significantly different (Figure 3-6). 


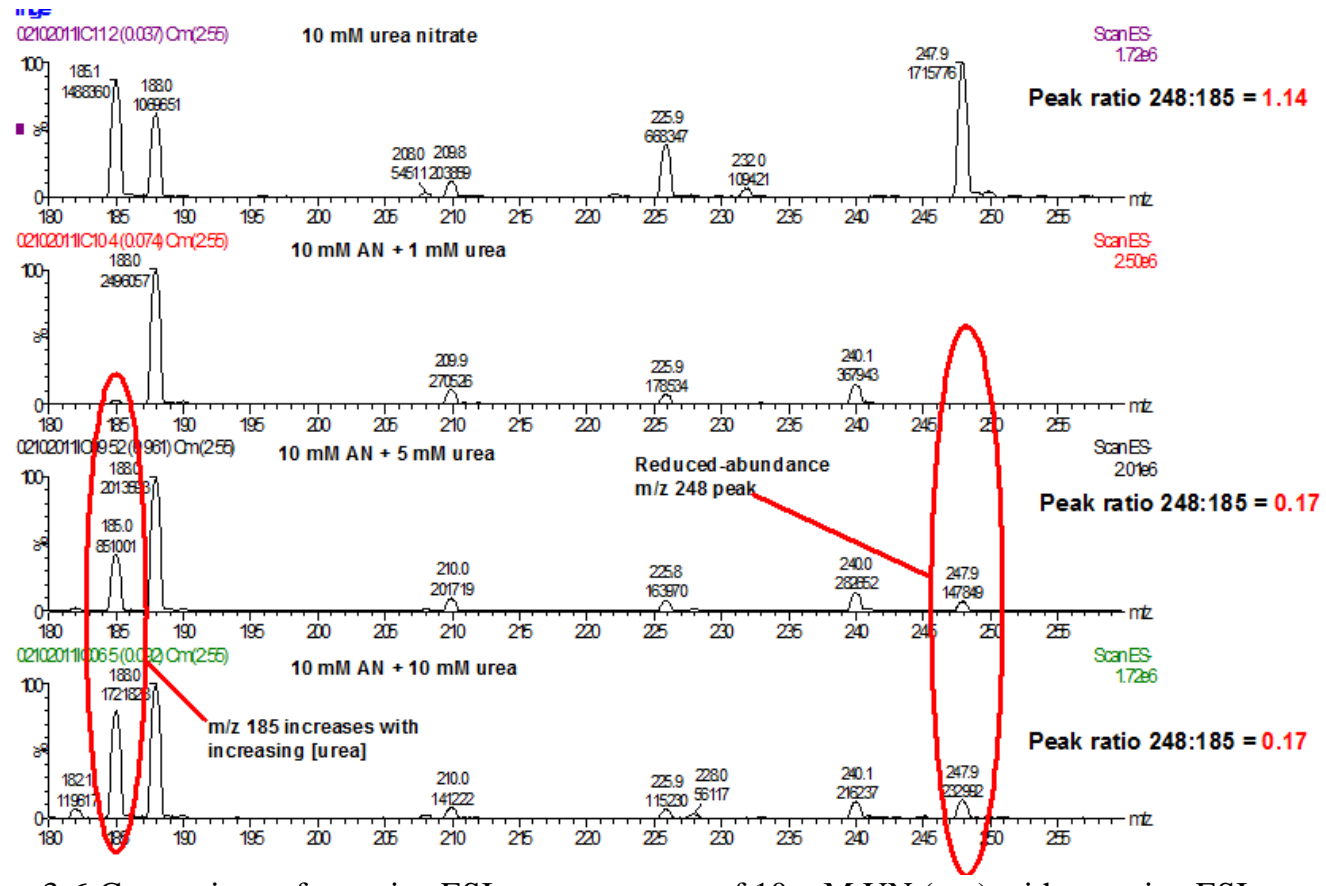

Figure 3-6 Comparison of negative ESI mass spectrum of $10 \mathrm{mM}$ UN (top) with negative ESI mass spectra of $10 \mathrm{mM}$ AN using varying amounts of urea.

Collision-induced dissociation and identification of the peaks at $\mathrm{m} / \mathrm{z} 182,185,188,242$, and 248 and for the mass spectrum of [urea+ $\left.\mathrm{NH}_{4} \mathrm{NO}_{3}\right]^{-}$was performed. When subjected to MS/MS analysis, fragmentation of $\mathrm{m} / \mathrm{z} 182$ peak gives the formula [2 urea $\left.+\mathrm{NO}_{3}\right]^{-}$ with a neutral loss of urea followed by loss of a second urea molecule and a proton (Figure 3-7).

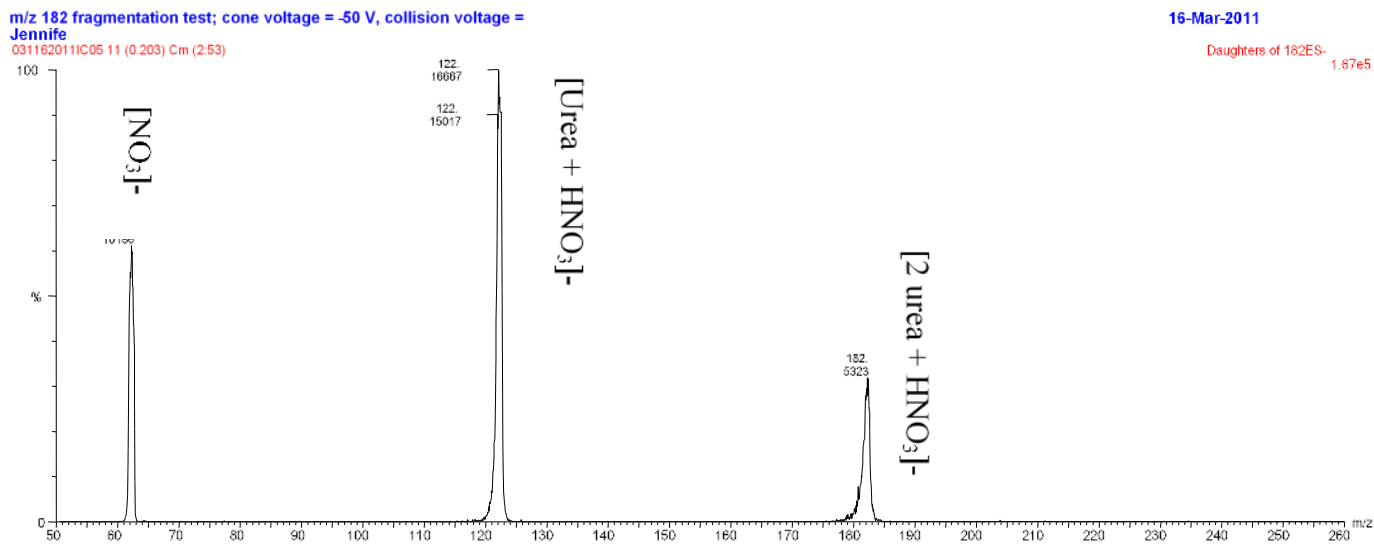

Figure 3-7. Collision-induced dissociation mass spectrometry (CID-MS/MS) was performed to determine the identity of the product ions of the $\mathrm{m} / \mathrm{z} 182$ peak. Identity and masses are labeled. 
The $\mathrm{m} / \mathrm{z} 185$ ion [urea nitrate $\left.+\mathrm{NO}_{3}\right]^{-}$, indicates the production of urea nitrate even though its presence in the samples composed of urea, ammonium nitrate, and a proton source is an artifact of the sample matrix and ionization conditions (Figure 3-8).

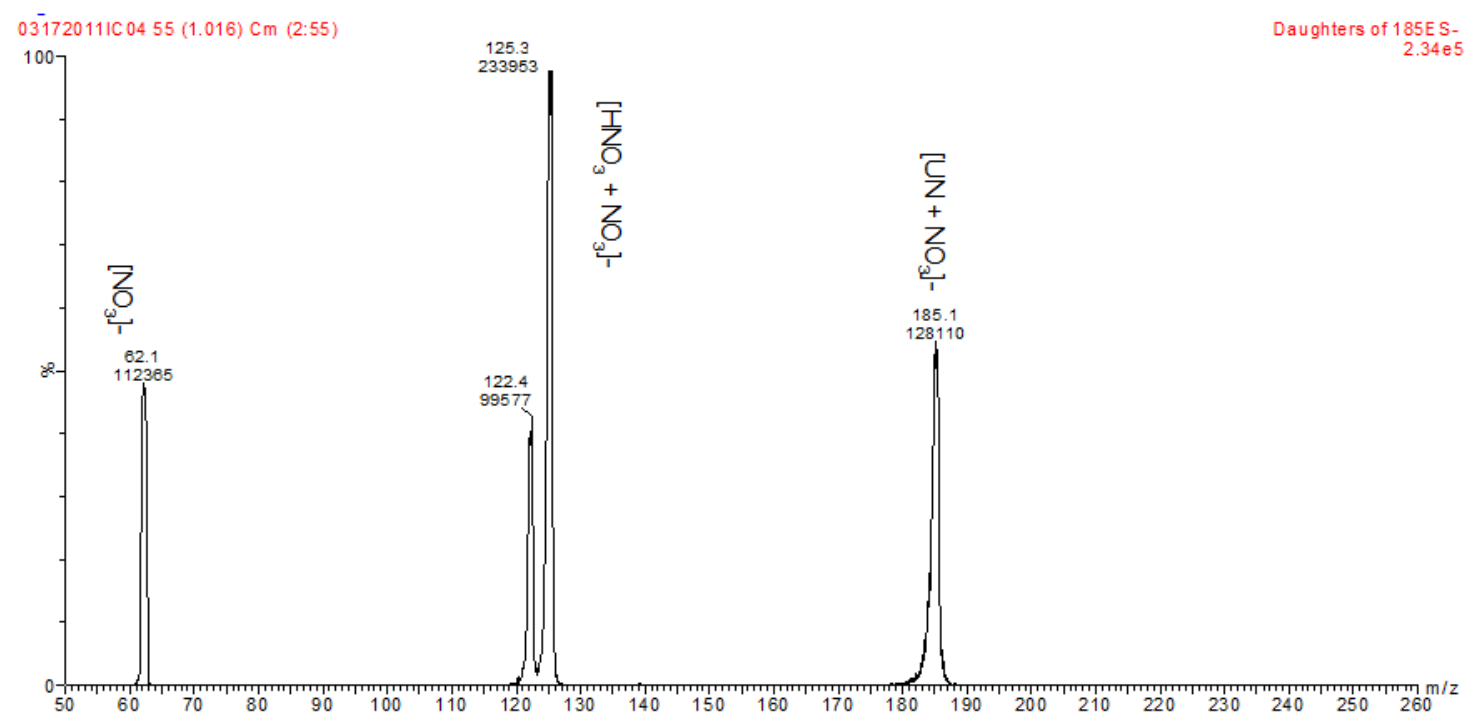

Figure 3-8. Collision-induced dissociation mass spectrometry (CID-MS/MS) was performed to determine the identity of the product ions of the $\mathrm{m} / \mathrm{z} 185$ peak. Identity and masses are labeled.

Fragmentation of the m/z 188 ion showed it to be $\left[2 \mathrm{HNO}_{3}+\mathrm{NO}_{3}\right]^{-}$. This ion was determined to hold no relevant information, as it is present in both true and conditionally generated UN, so it was not used further.

Fragmentation of the $\mathrm{m} / \mathrm{z} 242$ ion shows it to be a cluster ion containing three urea molecules and a nitrate ion $\left[3 \text { urea }+\mathrm{NO}_{3}\right]^{-}$. The $\left[3 \text { urea }+\mathrm{NO}_{3}\right]^{-}$ion is not present in a true UN sample (Figure 3-9). 


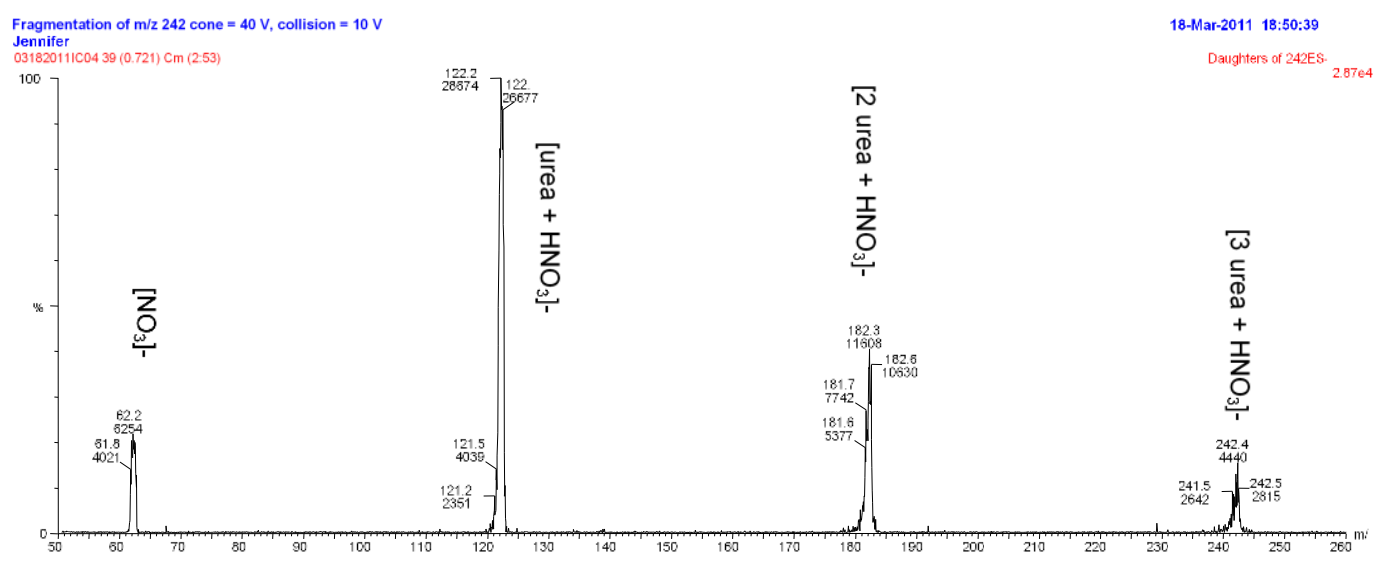

Figure 3-9. Collision-induced dissociation mass spectrometry (CID-MS/MS) was performed to determine the identity of the product ions of the $\mathrm{m} / \mathrm{z} 242$ peak. Identity and masses are labeled.

The $\mathrm{m} / \mathrm{z} 248$ ion corresponds to [urea nitrate $\left.+\mathrm{HNO}_{3}+\mathrm{NO}_{3}\right]^{-}$(Figure 3-10).

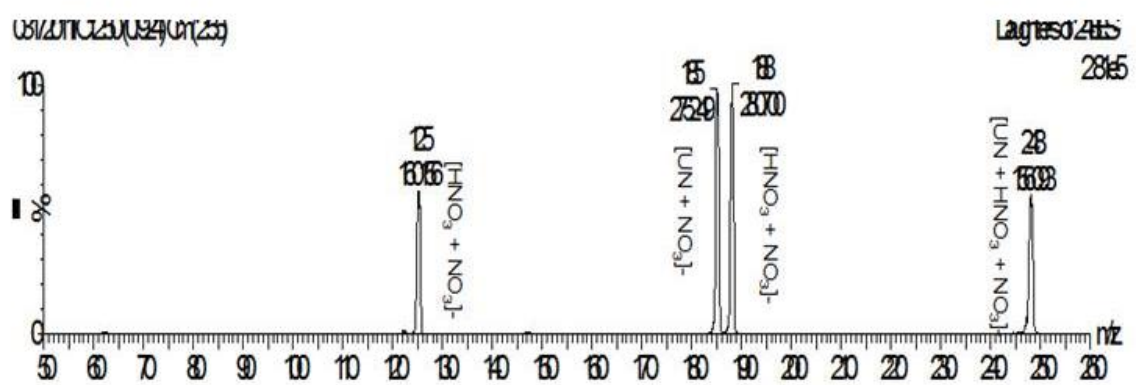

Figure 3-10. Collision-induced dissociation mass spectrometry (CID-MS/MS) was performed to determine the identity of the product ions of the $\mathrm{m} / \mathrm{z} 248$ peak. Identity and masses are labeled.

These experiments demonstrated that mixtures of urea and ammonium nitrate do contain an $\mathrm{m} / \mathrm{z} 185$ peak, suggesting the presence of the $\left[\mathrm{UN}+\mathrm{NO}_{3}\right]^{-}$adduct. However, the presence of a urea interference can be inferred by a strong $\mathrm{m} / \mathrm{z} 182$ peak with a relatively reduced $\mathrm{m} / \mathrm{z} 248$ ion $\left[\mathrm{UN}+\mathrm{HNO}_{3}+\mathrm{NO}_{3}\right]^{-}$, which contrasts with the obvious presence of the $\mathrm{m} / \mathrm{z} 248$ peak in true urea nitrate samples. The simple ratio of peak abundances (248:185) for each sample shows a much smaller value for mixtures of urea and 
ammonium nitrate when compared to true urea nitrate. Furthermore, as the concentration of ammonium nitrate in the sample increases relative to the concentration of urea, the abundance of the $\mathrm{m} / \mathrm{z} 182$ peak decreases.

For the next phase of this study, standards with $10 \mathrm{mM}$ ammonium nitrate and varying concentrations of urea $(1,5$, and $10 \mathrm{mM})$ were run and compared to the urea nitrate standard. A similar result was obtained; the abundance of the $\mathrm{m} / \mathrm{z} 185$ peak increased as urea concentration rose, and again the ratio of $\mathrm{m} / \mathrm{z} 248: \mathrm{m} / \mathrm{z} 185$ varied with the mixture ratio. Collision - induced dissociation permitted the identification of peaks for $\mathrm{m} / \mathrm{z} 182$, $185,188,242$, and 248 in the mass spectrum of urea plus ammonium nitrate. A summary of the CID - MS data of the urea-ammonium nitrate mixture compared to urea nitrate is shown in Table 3-3.

\begin{tabular}{|l|l|l|l|}
\hline Ion $\mathrm{m} / \mathrm{z}$ & Urea $+\mathrm{AN}$ mixture & Urea nitrate & Product ions \\
\hline $182\left[2 \mathrm{urea}+\mathrm{NO}_{3}\right]-$ & Present & Absent & 122,62 \\
\hline $185\left[\mathrm{UN}+\mathrm{NO}_{3}\right]-$ & Present & Present & $125,122,162$ \\
\hline $188\left[2 \mathrm{HNO}_{3}+\mathrm{NO}_{3}\right]-$ & Present & Present & 125,62 \\
\hline $242\left[3\right.$ urea $\left.+\mathrm{NO}_{3}\right]-$ & Present & Minimally present & $182,122,62$ \\
\hline $248\left[\mathrm{UN}+\mathrm{HNO}_{3}+\mathrm{NO}_{3} \cdot\right.$ Present & Present & $188,185,125$ \\
\hline Peak ratio, $248: 185$ & $<1.0$ & $>1.0$ & \\
\hline
\end{tabular}

Table 3-3. CID-MS peak data and product ion attribution.

Note that $\mathrm{m} / \mathrm{z} 182$ is not present and the $\mathrm{m} / \mathrm{z} 242$ ion is present only in vanishingly small amounts in the mass spectrum of the uncontaminated urea nitrate standard (Fig.3-11) when compared to the urea/AN mixture. 


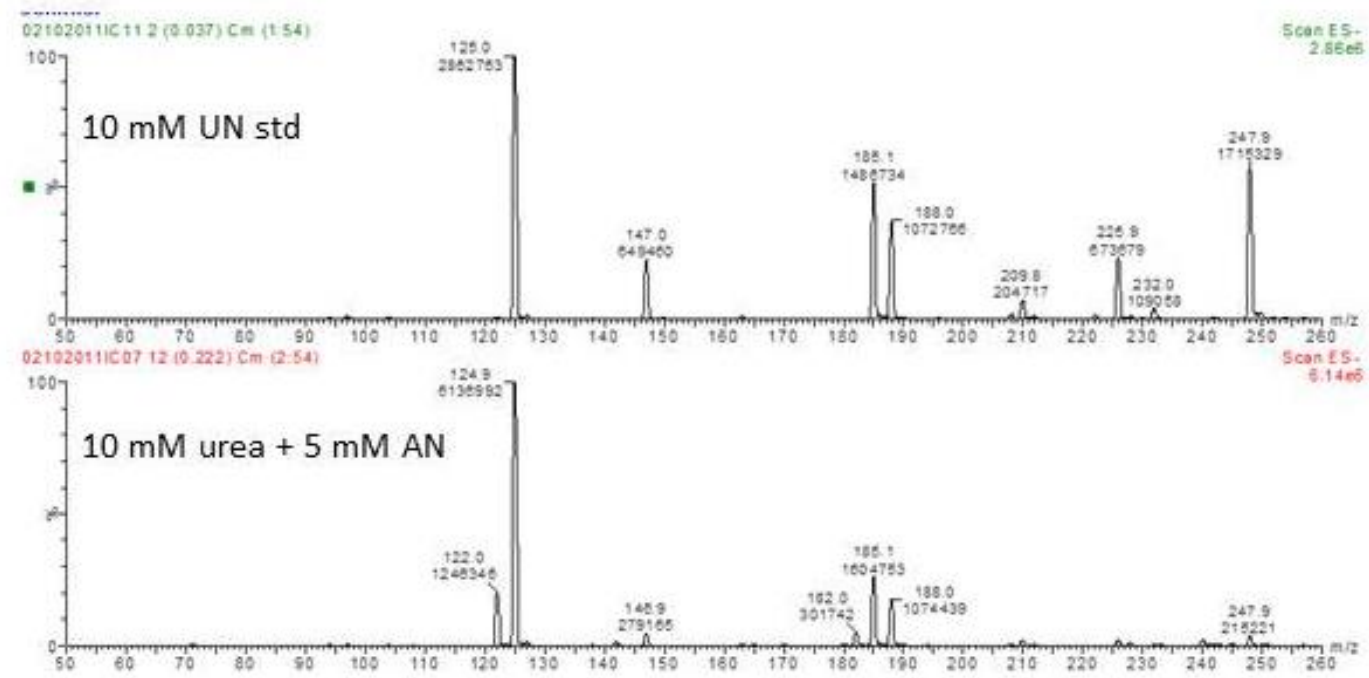

Figure 3-11. Top: $10 \mathrm{mM}$ UN standard. Bottom: $10 \mathrm{mM}$ urea $+5 \mathrm{mM}$ AN. Without a proton source, no UN signal is generated.

To determine whether the same compounds would form in the presence of a nitrate compound that was not a source of acidic protons, a sample with urea and potassium nitrate was prepared and analyzed (Fig. 3-12). In the absence of the source of acidic protons (top), neither the $\mathrm{m} / \mathrm{z} 185$ nor the $\mathrm{m} / \mathrm{z} 248$ peak are present.

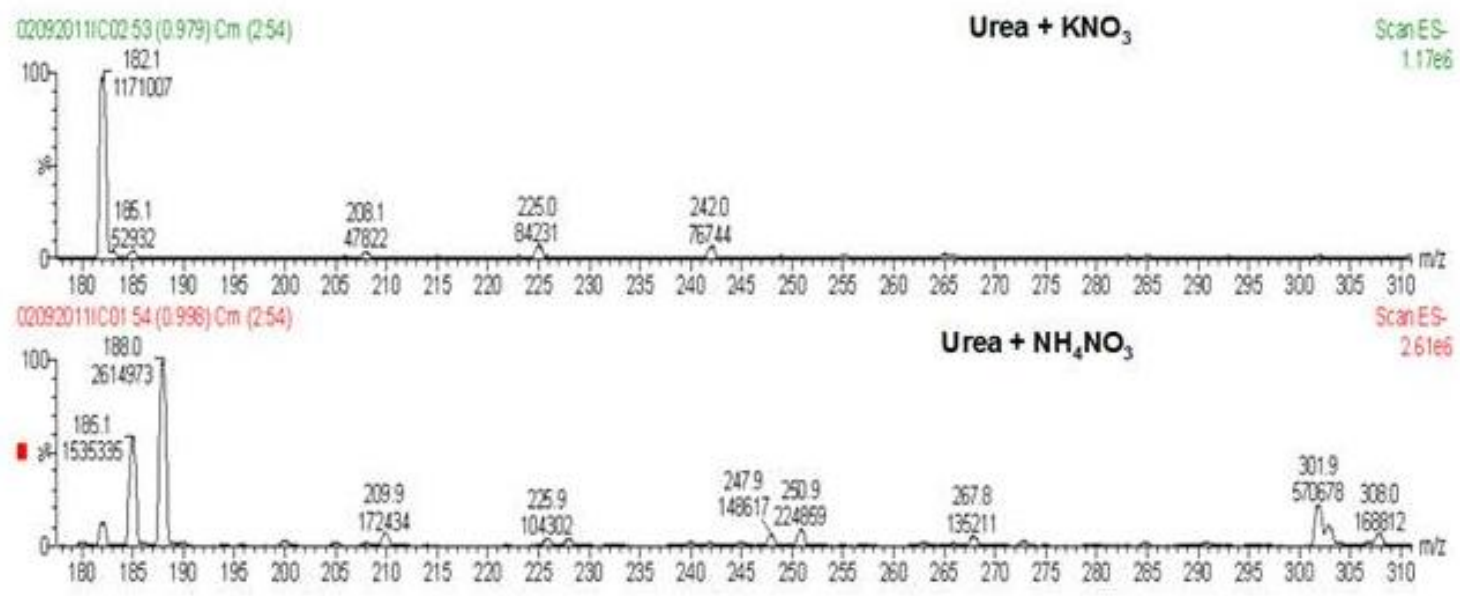

Figure 3-12. Top: $10 \mathrm{mM}$ urea $+10 \mathrm{mM} \mathrm{KNO} 3$. Bottom: $10 \mathrm{mM}$ urea $+10 \mathrm{mM} \mathrm{NH}_{4} \mathrm{NO}_{3}$. Without a proton source, no UN signal is generated. 


\section{Detection of Ammonium Nitrate and Urea Nitrate in the Presence of Various Interfering Compounds}

The ability to detect $\mathrm{AN}$ and $\mathrm{UN}$ on vehicles, clothing, or skin that may be contaminated with other substances such as fuels, oils, solvents, or other compounds is important because the locations in which these compounds may be collected are not always pristine. Therefore compounds were chosen that could reasonably be expected to be found on individuals or vehicles suspected of handling or transporting AN or UN. These compounds were added to AN and UN standard solutions and their effects on the target compounds determined under the same analysis conditions as the standards. The interfering compounds fell into five general categories: aqueous-based liquids, solvents, cleaning supplies, fuels, and lubricants.

$1 \mathrm{~mL}$ aliquots of $5 \mathrm{mM}$ of AN standard solution made as previously described were transferred to a set of sample vials. Each interfering compound was added to one sample vial and the mixture analyzed by the infusion method described previously. The interferent volume was chosen to produce a large enough change to observe its effects without overwhelming the sample signal. The process was then repeated with a $5 \mathrm{mM}$ UN standard. The results for both standards with each interferent are presented in Table 3-4 for AN and Table 3-5 for UN, and some qualitative conclusions are drawn. 


\begin{tabular}{|c|c|c|}
\hline Interferent & $\begin{array}{c}\text { Relative percent of } \\
\text { standard peak } \\
\text { height } \mathrm{m} / \mathrm{z} 178\end{array}$ & $\begin{array}{c}\text { Relative percent of } \\
\text { standard peak } \\
\text { height } \mathrm{m} / \mathrm{z} 258\end{array}$ \\
\hline $\begin{array}{l}5 \mathrm{mM} \text { AN standard } \\
\text { (reference) }\end{array}$ & 100 & 100 \\
\hline $5 \mu \mathrm{L}$ Citgo premium & 118 & 186 \\
\hline $5 \mu \mathrm{L}$ Mazola corn oil & 40 & 63 \\
\hline $5 \mu \mathrm{L}$ WD-40 & 44 & 72 \\
\hline $5 \mu \mathrm{L}$ marine diesel fuel & 50 & 137 \\
\hline $5 \mu \mathrm{L}$ Shell diesel fuel & 58 & 81 \\
\hline $5 \mu \mathrm{L}$ Carbonell olive oil & 45 & 63 \\
\hline $\begin{array}{l}5 \mu \mathrm{L} \text { absolute ethanol } \\
\text { (Pharmco - AAPER, lot } \\
\text { AS4001 }\end{array}$ & 75 & 111 \\
\hline $\begin{array}{l}5 \mu \mathrm{L} \text { Assured (Wal-Mart } \\
\text { brand) hand sanitizer }(62 \% \\
\text { EtOH) }\end{array}$ & 76 & 134 \\
\hline $\begin{array}{l}5 \mu \mathrm{L} \text { methanol (Fisher } \\
\text { Scientific lot } 100970\end{array}$ & 105 & 191 \\
\hline $\begin{array}{l}5 \mu \mathrm{L} \text { isopropanol (Acros } \\
\text { Organics, lot B0511904) }\end{array}$ & 107 & 195 \\
\hline $\begin{array}{l}5 \mu \mathrm{L} \text { BreakFree CLP } \\
\text { weapons lubricant }\end{array}$ & 105 & 195 \\
\hline $\begin{array}{l}5 \mu \mathrm{L} \text { Sweet's } 7.62 \text { solvent } \\
\text { weapons cleaner }\end{array}$ & 28 & -- \\
\hline $\begin{array}{l}5 \mu \mathrm{L} \text { Shooter's Choice FP- } \\
10 \text { lubricant }\end{array}$ & 28 & 28 \\
\hline $\begin{array}{l}5 \mu \mathrm{L} \text { Gatorade PerformO2, } \\
\text { orange flavor }\end{array}$ & 29 & 36 \\
\hline $5 \mu \mathrm{L}$ Diet Pepsi & 108 & 183 \\
\hline $5 \mu \mathrm{L}$ Windex glass cleaner & 28 & 41 \\
\hline $2 \mu \mathrm{L}$ Windex glass cleaner & 73 & 109 \\
\hline $\begin{array}{l}2 \mu \mathrm{L} \text { Great Value (Wal- } \\
\text { Mart brand) sodium } \\
\text { hypochlorite bleach }\end{array}$ & 11 & -- \\
\hline
\end{tabular}

Table 3-4. Analysis of AN with interfering compounds: $1 \mathrm{~mL} 5 \mathrm{mM}$ AN standard, $5 \mu \mathrm{L}$ interferent. Dashes indicate complete suppression of the ion. 


\begin{tabular}{|c|c|c|}
\hline Interferent & $\begin{array}{c}\text { Relative percent of } \\
\text { standard peak } \\
\text { height } \mathrm{m} / \mathrm{z} 185 \\
\end{array}$ & $\begin{array}{c}\text { Relative percent of } \\
\text { standard peak } \\
\text { height } \mathrm{m} / \mathrm{z} 248 \\
\end{array}$ \\
\hline $\begin{array}{l}5 \mathrm{mM} \text { UN standard } \\
\text { (reference) }\end{array}$ & 100 & 100 \\
\hline $5 \mu \mathrm{L}$ Citgo premium & 93 & 65 \\
\hline $5 \mu$ L Mazola corn oil & 93 & 80 \\
\hline $5 \mu \mathrm{L} \mathrm{WD}-40$ & 92 & 87 \\
\hline $5 \mu \mathrm{L}$ marine diesel fuel & 59 & 63 \\
\hline $5 \mu \mathrm{L}$ Shell diesel fuel & 81 & 111 \\
\hline $5 \mu \mathrm{L}$ Carbonell olive oil & 90 & 84 \\
\hline $\begin{array}{l}5 \mu \mathrm{L} \text { absolute ethanol } \\
\text { (Pharmco - AAPER, lot } \\
\text { AS4001 }\end{array}$ & 91 & 79 \\
\hline $\begin{array}{l}2.5 \mu \mathrm{L} \text { Assured (Wal-Mart } \\
\text { brand) hand sanitizer ( } 62 \% \\
\text { EtOH) }\end{array}$ & 119 & 169 \\
\hline $\begin{array}{l}2.5 \mu \mathrm{L} \text { methanol (Fisher } \\
\text { Scientific lot } 100970\end{array}$ & 57 & 83 \\
\hline $\begin{array}{l}5 \mu \mathrm{L} \text { isopropanol (Acros } \\
\text { Organics, lot B0511904) }\end{array}$ & 63 & 51 \\
\hline $\begin{array}{l}5 \mu \mathrm{L} \text { BreakFree CLP } \\
\text { weapons lubricant }\end{array}$ & 34 & 16 \\
\hline $\begin{array}{l}5 \mu \mathrm{L} \text { Sweet's } 7.62 \text { solvent } \\
\text { weapons cleaner }\end{array}$ & 14 & -- \\
\hline $\begin{array}{l}5 \mu \mathrm{L} \text { Shooter's Choice FP- } \\
10 \text { lubricant }\end{array}$ & 81 & 96 \\
\hline $\begin{array}{l}2.5 \mu \mathrm{L} \text { Gatorade } \\
\text { PerformO2, orange flavor }\end{array}$ & 80 & 46 \\
\hline $5 \mu \mathrm{L}$ Diet Pepsi & 41 & 44 \\
\hline $\begin{array}{l}2.5 \mu \mathrm{L} \text { Windex glass } \\
\text { cleaner }\end{array}$ & 88 & 57 \\
\hline $\begin{array}{l}2 \mu \mathrm{L} \text { Great Value (Wal- } \\
\text { Mart brand) sodium } \\
\text { hypochlorite bleach }\end{array}$ & 38 & 15 \\
\hline
\end{tabular}

Table 3-5. Analysis of UN with interfering compounds: $1 \mathrm{~mL} 5 \mathrm{mM} \mathrm{UN}$ standard, interferent concentration as noted. Dashes indicate complete suppression of the ion.

The peak abundances of the samples with interferents were normalized to the abundance

of the $\mathrm{m} / \mathrm{z} 178$ and $\mathrm{m} / \mathrm{z} 258$ peaks of the AN standard. With all interferents tested the $\mathrm{m} / \mathrm{z}$ 178 peak remained detectable although in some cases its abundance was significantly 
suppressed. In two cases the m/z 258 ion was completely suppressed. Both AN and UN decomposed on the addition of aqueous based interferent liquids. The effect was more pronounced for UN. Other ions present in the adulterating compound can also overwhelm any signal attributable to UN. While AN is more stable, aqueous liquids have a deleterious effect on the mass spectrum. Alcohol-based ammonia formulated window cleaners such as Windex show a slight effect on the UN signal at the time of addition and with the passage of time UN is slowly degraded because of the methanol in the glass cleaner. Addition of bleach results in the suppression of the UN signal and an enhancement of the $\mathrm{m} / \mathrm{z} 147$ peak as the $\left[\mathrm{NaNO}_{3}+\mathrm{NO}_{3}\right]^{-}$cluster is formed. Also enhanced is the $\mathrm{m} / \mathrm{z} 168$ peak, which may be the $\left[\mathrm{CaNO}_{3}+\mathrm{NO}_{3}\right]^{-}$cluster. Common motor fuels show minimal interference with the detection of UN and oils such as corn oil or canola oil do not interfere with the detection of UN. Petroleum-based weapons cleaning compounds show an effect on AN similar to fuels, reducing the peak abundance. Both compounds were severely affected by Sweet's 7.62 work bore cleaning solvent, which contains 5\% ammonia and results in the loss of signal for both AN and UN. The individual sample runs for AN and UN with the different interferents are collected in the Appendix.

\section{Conclusions}

In this section a method for the determination of urea and ammonium nitrate by nonaqueous electrospray MS/MS is described. Examination of the mass spectral data allows the differentiation of true urea nitrate from samples containing mixtures of urea, a nitrate salt, and an acidic proton source. Even though both contain a peak at m/z 185 
corresponding to the [urea nitrate $\left.+\mathrm{NO}_{3}\right]^{-}$cluster, the AN/urea mixture also shows peaks at $\mathrm{m} / \mathrm{z} 182$ and $\mathrm{m} / \mathrm{z} 242$ due to the presence of cluster ions containing urea that are not present in a mass spectrum of true urea nitrate. Additionally, the $\mathrm{m} / \mathrm{z} 248$ peak corresponding to [urea nitrate $\left.+\mathrm{HNO}_{3}+\mathrm{NO}_{3}\right]^{-}$will be substantially reduced in a mixed sample. Taking a simple ratio of the $\mathrm{m} / \mathrm{z} 248: 185$ peaks also aids in the determination of whether a sample contains urea nitrate. For true UN samples the ratio is greater than 1.0 and for mixtures the ratio is approximately 0.2 . If the mixture does not have an acidic proton source present, the $\mathrm{m} / \mathrm{z} 185$ ion is not produced. Interfering compounds show a wide variety of influences on urea nitrate. Oils and fuels have minimal effect while aqueous liquids and cleaning products have detrimental and complicating effects on the mass spectrum. Overall this procedure provides a rapid method for quickly screening for the presence of these improvised explosives on a variety of surfaces.

\section{References}

[1] Blasters' Handbook, Explosive Products Division, E. I. Du Pont de Nemours \& Co., Inc., Wilmington, Delaware, $16^{\text {th }}$ edition, 1980, p.59.

[2] Marshall, M., Oxley, J.C., (Eds.), Aspects of Explosives Detection, Elsevier B.V., 2009.

[3] NDTV, Delhi court blast: 11 dead, 74 injured; sketches of suspect released. Available from: http://www.ndtv.com/article/india/delhi-court-blast-11-dead-74-injured-sketchesof-suspect-released-132067 (accessed 18 August 2012).

[4] Almog, J., Burda, G., Shloosh, Y., Abramovich-Bar, S., Wolf, E., Tamiri, T., J. Forensic Sci. 52(2007), 128.

[5] Woodfin, R.L., Trace Chemical Sensing of Explosives, John Wiley \& Sons Inc., Hoboken, NJ, 2007.

[6] Strategypage, Afghanistan bans explosive fertilizer. Available from: http://www.strategypage.com/htmw/htweap/20100125 (accessed 03 March.2011). 
[7] aware eZine Gamma, Improvised Urea Nitrate. Available from: http://www.awarenetwork.org/etc/gamma/?x=5 (accessed 15 June 2012).

[8] ShadowRx Forums, Urea Nitrate. Available from:

http://www.shadowrx.com/forums/showthread.php?t=976 (accessed 15 June 2012).

[10] Zhao, X., Yinon, J., Rapid Commun. Mass Spectrom. 15 (2001) 1514.

[9] Buczkowski, D., Zygmunt, B., Propellants, Explos., Pyrotech. 32 (2007) 411.

[11] Henderson, I.K., Saari-Nordhaus, R., J. Chromatogr. 602 (1992), 149

[12] Johns, C., Shellie, R.A., Potter, O.G., O'Reilly, J.W., Hutchinson, J.P., Guijt, R.M., Breadmore, M.C., Hilder, E.F., Dicinoski, G.W., Haddad, P.R., J. Chromatogr. A 1182 (2008) 205

[13] Hutchinson, J. P., Evenhuis, C. J., Johns, C., Kazarian, A.A., Breadmore, M.C., Macka, M., Hilder, E.F., Guijt, R.M., Dicinoski, G.W., Haddad, P.R., Anal. Chem. 79 (2007), 7005.

[14] Cheng, S., Dou, J., Wang, W., Chen, C., Hua, L., Zhou, Q.H., Hou, K.Y., Li, J.H., Li, H.Y., Anal. Chem. 85 (2013) 319.

[15] Deb, M. K., Verma, D., Verma, S.K., Bodhankar, N., Sircar, J.K., J. Indian Chem. Soc. 87 (2010) 1317.

[16] Hall, K., McCord, B.R., J. Forensic Sci. 38 (1993), 928.

[17] Almog, J., Klein, A., Tamiri, T., Shloosh, Y., Abramovich-Bar, S., J. Forensic Sci. 50 (2005) 582.

[18] Harkema, S., Feil, D., Acta Cryst B 25 (1969), 589.

[19] Beveridge, A., (Ed.), Forensic Investigation of Explosions, $2^{\text {nd }}$ ed., CRC Press, Boca Raton FL, 2012.

[20] Cagan, A., Lu, D., Cizek, K., LaBelle, J., Wang, J., Analyst 133 (2008) 585.

[21] Tamiri, T., Rozin, R., Lemberger, N., Almog, J., Anal. Bioanal. Chem. 395 (2009) 421.

[22] dePerre, C., McCord, B.R., Forensic Sci. Int. 211 (2011) 76.

[23] Almog, J., Espino, D., Tamiri, T., Sonenfeld, D., Forensic Sci. Int. 224 (2013) 80.

[24] Tamiri, T., Rapid Commun. Mass Spectrom. 19 (2005) 2094. 
[25] dePerre, C., Prado, A., McCord, B.R., Rapid Commun. Mass Spectrom. 26 (2012) 154.

[26] Wynn, C.M., Palmacci, S., Kunz, R.R., Rothschild, M., Opt. Express 18 (2010) 5399.

[27] Guha, C., Chakraborty, J.M., Karanjai, S., Das, B., J. Phys. Chem. B 107 (2003) 12814.

[28] 2-Methoxyethanol, SDS number 284467, Sigma-Aldrich. Available from: http://www.sigmaaldrich.com/catalog/product/sial/284467?lang=en \&region=US, December12, 2012 (accessed 12 May 2012).

[29] Lahoda, K.G., Collin, O.L., Mathis, J.A., LeClair, H.E., Wise, S.H., McCord, B.R., J. Forensic Sci, 53:4 (2008), 802. 


\section{CHAPTER 4: Development of a Paper Microfluidic Device for the Detection of Improvised Explosives}

\section{A. Introduction}

Paper microfluidics is a transformational technology that permits the development of very inexpensive analytical devices based on designs printed in wax-based ink on chromatography paper. The wax channels in the paper direct liquid samples toward individual sections of the paper containing colorimetric test reagents. With proper design, a single device can perform five or more simultaneous analyses while costing only pennies, since the basic design components—chromatography paper, wax, and small quantities of reagents - are all very inexpensive. The paper devices are easily stored for long-term performance and because of their low cost could revolutionize on-site forensic testing.

Overall, the development of this paper based sensor will provide police and forensic evidence collection teams an easily-stored and reliable tool for presumptive testing of unknown evidence. Sheets of these paper devices could be easily and cheaply manufactured and take up no more space than a package of cigarettes. Their low cost and easy portability would mean that every analyst and crime scene response team member could carry a kit capable of instant identification of unknown or hazardous compounds.

Improvised or homemade explosives (HMEs), which were once limited to war zones, have now become a concern for first responders and law enforcement in the United States who may encounter organized groups or curious individuals synthesizing HMEs. In 
these situations, fast and accurate identification of the explosive compound used is of the utmost importance. Common constituents of HMEs include organic and inorganic chemicals in the molecular form and as ions, sugars, and elemental metals. Although many different analytical technologies exist for detecting and quantifying HMEs, the different unregulated and easily obtained organic and inorganic compounds used in the devices vary greatly in mass, structure, and physicochemical properties with no single analytical instrument having the capability to identify them all.

A number of different analytical techniques are available for the identification of HMEs. GC/MS, LC/MS, or FT-IR may be used for organic compounds and sugars while IC and $\mathrm{CE}$ are used for inorganic ions $[1,2,3]$. Metals may be detected by scanning electron microscopy/ elemental diffraction spectroscopy (SEM/EDS) or by X-ray diffraction [7.8]. Some compounds require infusion ESI-MS to be identified in a sample $[4,5,6]$. These detection techniques have similar limitations: they are large and costly pieces of instrumentation that, with the exception of FTIR, are not portable. Since power, vacuum, and gas requirements mean these instruments are necessarily centrally located it follows that the sample must be collected following chain of custody requirements and brought to the laboratory, thereby increasing the amount of time before any analytical information on the identity of the explosive can be provided to first responders.

Additionally, samples may need to go through preparative techniques such as filtration or extraction before instrumental analysis can be performed. This adds to the total analysis time and has the potential for the analytes to be lost through such processes as adsorption onto the filtration medium or degradation of the sample by interaction with the extraction 
solvent or the surrounding atmosphere. Samples must also be prepared in sufficient volume (generally about $200 \mu \mathrm{l}$ per instrument) to be handled by an autosampler.

Organic and inorganic ions and compounds, sugars, and metals are all seen in HME analysis but no single instrument can detect all these groups. Instead, an unknown sample must be taken through a series of extractions for analysis by different types of instrumentation. The goal of this project is to develop a paper microfluidic device that can be used as an immediate-response field screening tool for improvised explosives and their constituent ions using minimal sample $(<50 \mu \mathrm{l})$ and can be used by personnel in the field with minimal training.

Current literature presents examples of paper microfluidic devices developed as healthcare diagnostic tools to be used in developing nations that do not possess the infrastructure to maintain typical diagnostic equipment [7]. These principles were adapted for the practical application of paper microfluidic devices to the screening of improvised and homemade explosives.

\section{B. Background}

Microfluidics involves the manipulation of fluids on a very small (nanoliter) scale. Traditional microfluidic techniques use glass or polydimethyl siloxane (PDMS) chips hooked to micro-scale pumps, valves, and power systems to transport the sample and effect the separation before reaching a detector such as a laser. By miniaturizing the system and exploiting the properties of fluids at the micro level, the sample volume is reduced, the attendant reagent volume is similarly reduced, and the overall efficiency of 
the system is increased [8]. DNA analysis and "lab-on-a-chip" technologies used in microbiology and proteomics are two of the biggest beneficiaries from fundamental microfluidics research.

PDMS chips must be painstakingly fabricated, resulting in a high attendant cost per chip, and they must be handled carefully to avoid breakage. The need for a power system to drive the separation and detect the sample components means a traditional microfluidic system can only be used where electricity and a controlled environment are available.

Microfluidic principles have been applied to paper substrates to create paper microfluidic devices (PMDs). A wax pattern is printed onto the surface of filter paper or chromatography paper and the paper is heated so the liquid wax will penetrate the paper, creating hydrophobic barriers that function as lanes for the liquid sample to move along as it is routed to multiplexed detection zones. The natural wicking action of the paper as it draws up the liquid sample takes the place of a pump, and test reagents placed precisely within the pattern give an immediate, visible color change showing the presence of an analyte. The same principle is used in such diagnostic products as home pregnancy test kits and urine glucose monitoring strips.

PMDs have been produced a number of ways, but they all have in common the creation of hydrophobic channels directing the sample to the test reagent. Substrates have included filter paper, chromatography paper, and nitrocellulose membrane $[9,10,11]$, all of which have worked well. Fabrication methods have included plasma etching [12], photolithography [13, 14,], polydimethylsiloxane (PDMS) printing [15], and wax printing $[9,11]$. While PMDs made by the wax printing process are not as finely resolved as 
those made using photolithography, they do not require the specialized equipment and personnel trained in the maintenance and use of that equipment that the other processes do [13].

Recently PMDs have found a use as healthcare diagnostic devices in developing regions of the world that do not have the infrastructure needed to support traditional diagnostic equipment The PMD with its colorimetric tests is administered and the results photographed with the camera of a mobile phone. The image, along with images of standards run in the field, is transmitted to an expert in a laboratory who evaluates the results and responds with a course of treatment if it is required [13].

Colorimetric spot tests are a well-established, fundamental technique that has been used for about a hundred years in various forms. Using physical and chemical properties of the chosen analyte, spot tests react to give a visible color change when the analyte of interest is present. Color spot tests may be presumptive or specific to a functional group, and are used in the analysis of explosives, bulk narcotics, and bodily fluids. They may test for intact compounds, ions or functional groups, metals, or enzymes $[16,17]$. They may be based on classical wet chemistry methods [16], nanoparticle aggregation [18], or antibody/aptamer detection. Color test kits for the analysis of nitroaromatics, nitrate esters, and nitramines using spray reagents are commercially available [19]. Spot tests found in the literature for such compounds as urea nitrate [20] and hydrogen peroxide $[21,22]$ may be adapted to the PMD format.

By using multiple indicators for the same compound, the user's ability to discriminate between different shades and intensities of color that indicate concentration is increased 
and data is more accurately interpreted [23]. For ions and small molecules, multiple indicators for the same compound may also help avoid a false positive result since the interferences for tests that use different reagents or mechanisms for the same analyte are unlikely to be the same for both systems.

These devices could be used in an emergency management or military context. An aggregation of the literature surrounding these two technologies, combined with preliminary results, shows the feasibility of using them to detect improvised and homemade explosives. The combination of these two well-established technologies, coupled with the concerns of first responders and forensic professionals that improvised and homemade explosives may be encountered in routine work, led to the belief that it is worthwhile to explore the development of a paper microfluidic device for the analysis of improvised and homemade explosives. The challenge of detecting a wide variety of compounds with very dissimilar properties is a suitable application to test this technique. The use of PMDs in the field with all their attendant benefits - speed, low cost, portability, specificity, sensitivity, ease of use and ability to generate results without electricity or specialized equipment — is limited only by the choice of compounds one wishes to detect.

\section{Goals of This Project}

The goal of this project is to develop and validate a paper microfluidic device (PMD) capable of providing fast and sensitive colorimetric detection of the constituents of improvised or homemade explosives in pre- or post-blast settings. The PMD is to be

used as a first-line response and preliminary field identification tool for the presence of 
improvised or homemade explosives before samples are sent to a central laboratory for analysis. This project has three objectives: first, using colorimetric detection techniques, determine the most sensitive and visually striking indicators for each analyte. Built-in redundancy using different tests for the same analyte may be employed to reduce the risk of false positives.

The second part of the study will be to design the PMD configuration that yields the greatest amount of sample information using the smallest amount of sample in a minimal extraction volume. The final part of this study will be to show proof of concept using the test reagents embedded in the PMD to give a fast, visible response showing the presence of compounds and ions present in HMEs.

\section{Development of the Paper Microfluidic Chip}

A simple single-loop PMD test device (Figure 4-1) was designed using Microsoft Paint and printed using a wax ink printer (Xerox Color Qube 8570; Xerox Corporation, Norwalk CT, USA). A sheet of the devices was printed on Whatman \#1, grade 1 (0.18 $\mathrm{mm}$ thickness) chromatography paper. One sheet of this paper measures 46 x $57 \mathrm{~cm}$ which, when cut carefully, yields 4 standard-size $81 / 2$ x 11 sheets on which the PMD can be printed. A piece of aluminum foil was folded over the printed sheet and used as a carrier when the sheet was run through a temperature-controlled document laminator three times at $160^{\circ} \mathrm{C}$ using the slowest speed. The foil carrier is used to ensure that the wax ink soaks into the paper to form the channels that direct the liquid sample to the test areas and that the wax ink is not transferred to the rollers of the laminator. The paper in its foil carrier is allowed to cool. The foil is then peeled away and the finished sheet is 
cut into individual devices. Total sample migration distance is $32 \mathrm{~mm}$ and the width of the sample lane is $3.5 \mathrm{~mm}$.

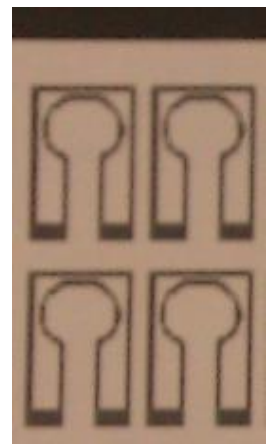

Figure 4-1. Closeup of single test loops.

The test devices were originally printed using black ink. During testing it was observed that organic solvents caused the color in the wax ink to bleed and sometimes obscure test results. The printer manufacturer was contacted about the possibility of purchasing white or colorless ink, but this was unavailable so the ink colors were tested to determine which migrated or interfered with color tests the least. It was ultimately determined that cyan ink was the best color for the PMDs, because the color migrated the least and was still able to provide a solid barrier to solvent flow.

A five-test PMD chip (Figure 4-2) was also designed using MS Paint. The dimensions are approximately $30 \mathrm{~mm}$ wide by $25 \mathrm{~mm}$ high. The device is shown below. Red food coloring in water was used to mark the sample migration area.

Figure 4-2. Five-test paper microfluidic device.

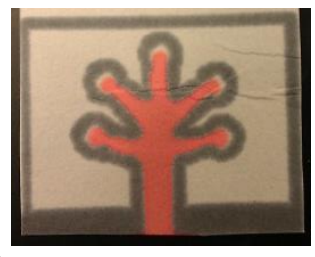




\section{E. Choice of Color Tests}

Five analytes were initially chosen for the first PMD. Urea nitrate, peroxide compounds, ammonium ion, nitrate ion, and nitrite ion were chosen as target analytes. The tests chosen are outlined below.

\section{Urea Nitrate}

para-dimethylaminocinnamaldehyde ( $p$-DMAC; Acros Organics, NJ, USA) has previously been shown to detect the uronium (protonated urea) ion [20] and has been used as a color indicator in other analyses for urea nitrate [6]. $20 \mu \mathrm{L}$ of a solution of pDMAC in ethanol was carefully spotted into the loop of the test device and allowed to dry. The open end of the test device was carefully lowered into a solution of urea nitrate in methanol and the liquid sample was drawn into the test device by capillary action. The red color characteristic of the interaction of $p$-DMAC with the uronium ion was formed (Figure 4-3).

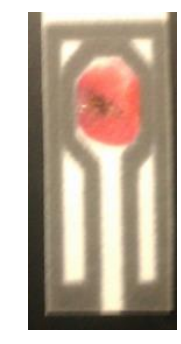

Figure 4-3. Positive result for p-DMAC reagent with urea nitrate dissolved in methanol and run immediately after being put into solution.

The mechanism of color formation was previously outlined in chapter 3 (p. 68). While acetone is a better solvent for urea nitrate, it was found that even though the appropriate color was formed, acetone causes the dye in the black ink to be solubilized and migrate to 
the top of the test strip, potentially obscuring faint positive results. When methanol is used, the test must be performed as soon as the sample is put into solution as urea nitrate dissociates in methanol in a relatively short time.

\section{Peroxide-containing Compounds}

For peroxide-containing compounds including TATP, HMTD, and hydrogen peroxide, ammonium titanyl oxalate, ATO (Acros Organics, NJ, USA) was used. A saturated solution in DI water was spotted into the loop of the test strip and allowed to dry before testing. The reagent was tested with a solution of $37 \%$ hydrogen peroxide (Figure 4-4) and yielded a bright yellow color. When TATP was dissolved in 1:1 acetone and methanol for the test, a less-intense yellow color (Figure 4-5, methanol blank shown for comparison) was observed. The mechanism for the reaction is outlined in Figure 4-6.
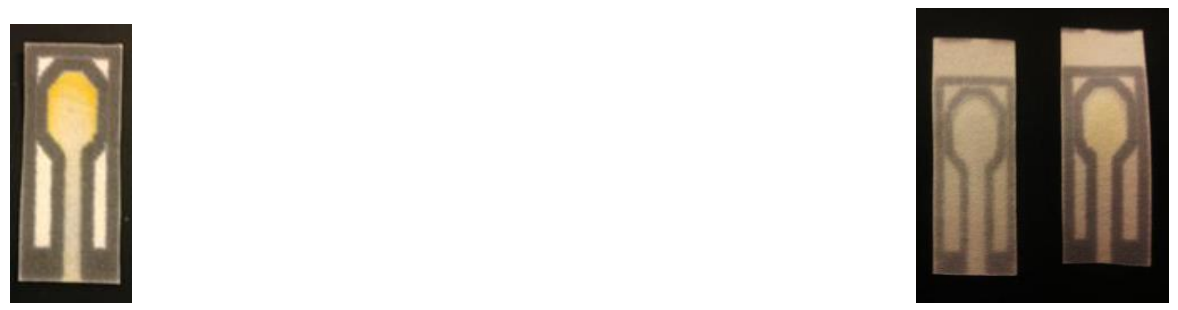

Figure 4-4. ATO test, 37\% hydrogen peroxide.

Figure 4-5. ATO test, TATP (blank left, TATP right)
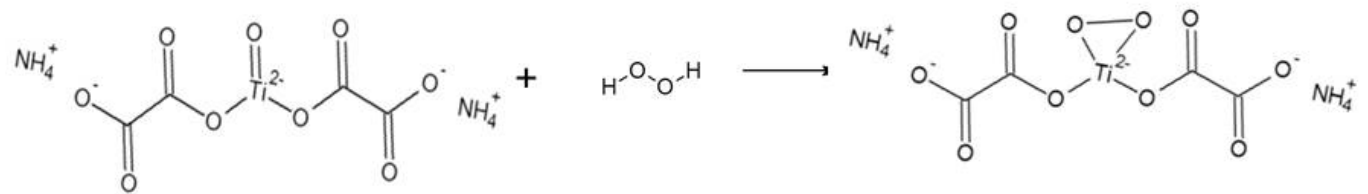

Figure 4-6. Color formation mechanism of ATO with hydrogen peroxide. 


\section{Nitrate Ion}

For nitrate analysis, the method outlined by Niki et al. [26] was chosen as the basis of this test. This is a spot test for the detection of nitrates and nitrites in wastewater. There are three reagents used in this method. The first is a solid reducing mixture of sulfanilic acid, sodium acetate, and powdered zinc that is added to the sample. The second reagent is concentrated sulfuric acid, and the third is $2.5 \%$ 1-naphthol in ethanol. An optional final step using $20 \% \mathrm{NaOH}$ to change the final positive color result from orange to bright pink was deemed unnecessary and omitted because the color change on the PMD when $\mathrm{NaOH}$ was applied was minimal.

To build the test loop for this analyte, $2.5 \mu 1$ of saturated trehalose solution in ethanol was applied to the bottom of the sample lane. The purpose of the trehalose is to hold the powdered reducing agent and to slow the movement of the sample up the channel [27], allowing it to react with the reducing mixture. Midway up the lane, $5.0 \mu \mathrm{l}$ of $20 \% \mathrm{H}_{2} \mathrm{SO}_{4}$ was pipetted and allowed to dry. Finally, $5.0 \mu \mathrm{l}$ of 1 -naphthol in ethanol was pipetted in the loop of the sample test strip and allowed to dry. In this test the nitrate is reduced to nitrite and a positive result is an orange color (Figure 4-7). The mechanism of the reaction is shown in Figure 4-8

Figure 4-7. Nitrate test result. Modified from Niki et al. [26] 
The $2.5 \mu$ l of saturated trehalose solution pipetted into the sample lane (width approximately $3.5 \mathrm{~mm}$ ) slowed the total migration time by approximately 100 seconds. It should be noted that no degradation of the chromatography paper was noted over the course of 36 hours where the $5.0 \mu \mathrm{l}$ of $20 \% \mathrm{H}_{2} \mathrm{SO}_{4}$ solution was applied.

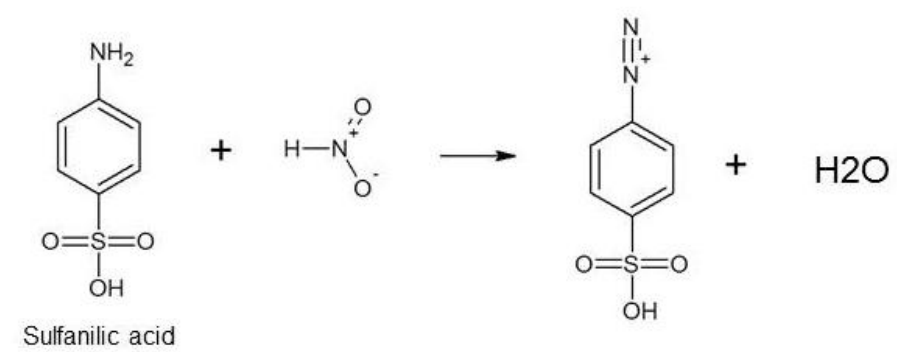

Nitrous acid
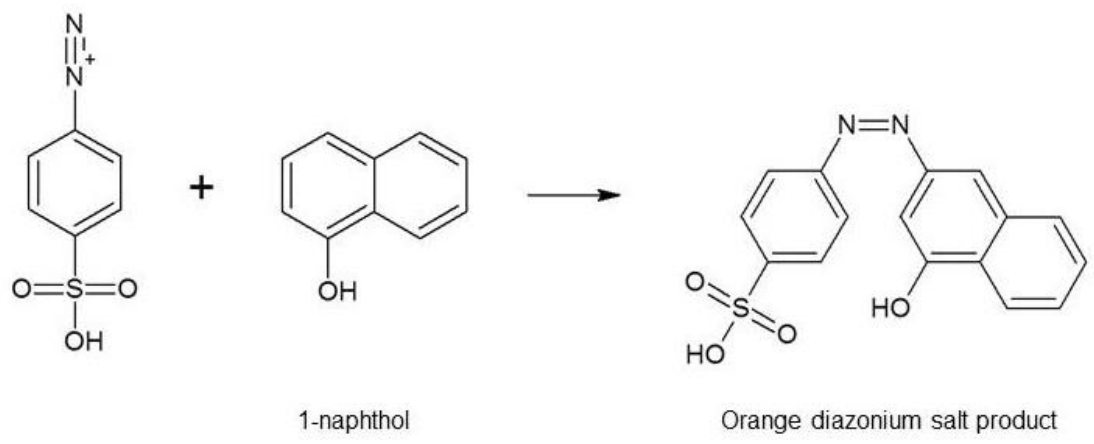

Orange diazonium salt product

Figure 4-8. Color formation mechanism for the nitrate ion test.

\section{Nitrite Ion}

For the nitrite ion test, Griess reagent was prepared by spotting $0.5 \%$ aqueous 1 naphthylamine (Sigma-Aldrich, St. Louis, MO, USA) midway up the sample lane and $0.1 \%$ aqueous sulfanilic acid at the top of the sample lane. An orange line revealed a positive result for this test (Figure 4-9). 


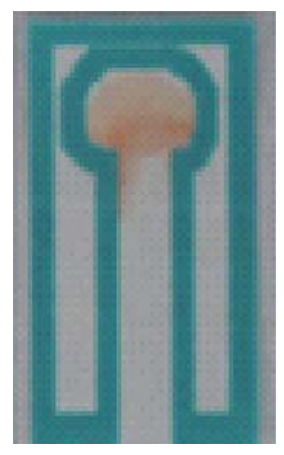

Figure 4-9. Nitrite test result using 1-naphthylamine.

The mechanism of the reaction is outlined in Figure 4-10.

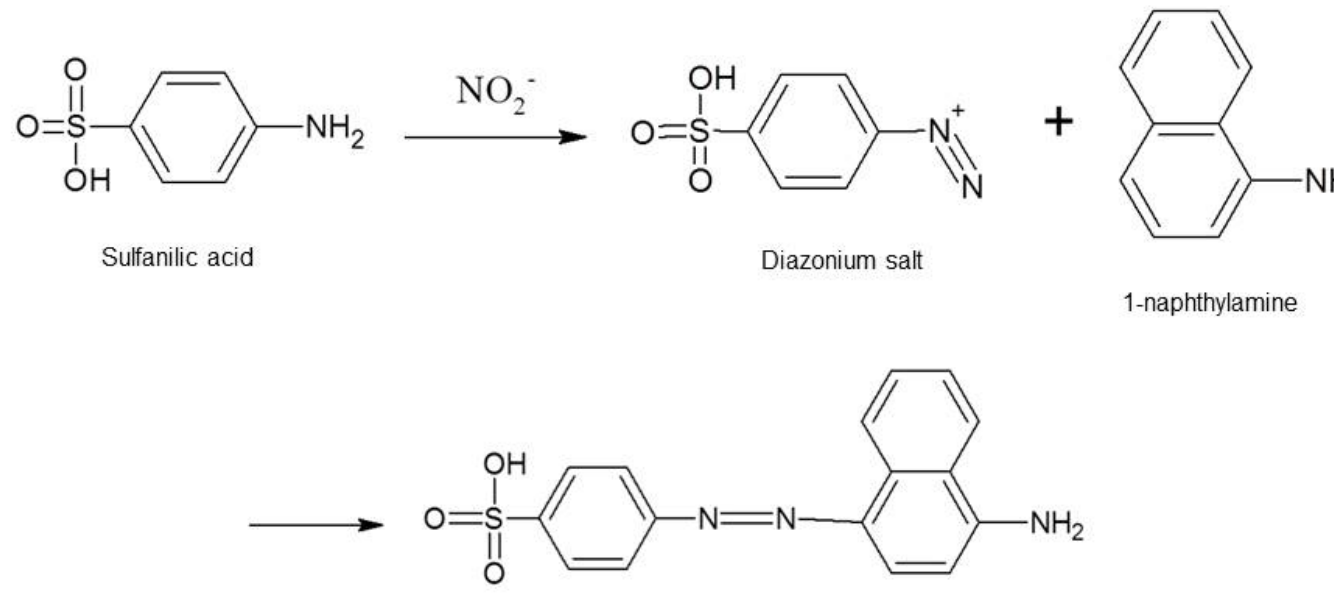

Orange-colored azo dye product

Figure 4-10. Color formation mechanism for the nitrite ion test.

After the development of tests for nitrate and nitrite ions, a study was performed to determine whether nitrate and nitrite ions could be differentiated using this PMD. When only nitrite was present, the nitrite lane appeared orange/brown and the nitrate lane was purple, presumably because of the reduction of nitrite. When only nitrate was present, an orange color appeared in the nitrate lane and the nitrite lane gave no result. When both 
nitrate and nitrite were present both channels are colored, with an orange/brown color in the nitrite lane and orange and purple colors in the nitrate lane. The device successfully differentiated between nitrate and nitrite based explosives and detected the presence of nitrite in post-blast samples from the reduction of nitrate.

\section{Ammonium ion}

For ammonium ion, two tests were evaluated. The first was based on a wastewater analysis method for ammonium ion used by Hori et al [25]. It uses a diazo coupling with a phenol followed by color development catalyzed using powdered boric acid. In this procedure, $0.1 \mathrm{~g}$ powdered boric acid and $0.1 \mathrm{~g}$ 2-phenylphenol are added to $10 \mathrm{ml}$ of sample. $0.1 \mathrm{~g}$ of dichloroisocyanuric acid (DCI) is then added and the sample is shaken. 5 $\mathrm{ml}$ of $1 \mathrm{~N} \mathrm{NaOH}$ is added and the sample shaken again to change the $\mathrm{pH}$ and generate the final color change to green. In this method the solution is drawn up a strip of nonwoven fabric coated with stripes of benzylcetyldimethylammonium chloride (BCDMA), which holds the colored reaction product. The number of colored stripes present after drawing the sample through indicates the concentration of ammonium ion present in the sample.

In the attempt to modify this test to the paper substrate, 1-naphthol was used in place of 2-phenylphenol because it was readily available. The test was first run using solutions of ammonium nitrate in deionized water, acetone, methanol, and the $95 \%$ acetone $/ 5 \%$ methyl cellosolve reagent used for ammonium nitrate and urea nitrate ESI-MS infusion experiments (Chapter 3). The best color development occurred in DI water, but a light blue color also developed in acetone and the 95/5 reagent. 
A small amount of BCDMA was added to the positive sample in DI water and shaken. Upon standing, the blue-green color was concentrated in a thin layer on the surface of the liquid and the remaining solution was decolorized (Figure 4-11).

An alternate procedure [24] was also evaluated. This procedure used DCI with a solution of $2.41 \%$ manganese sulfate in DI water and 20\% 1-naphthol in EtOH solution. This procedure was tested in solution and generated a positive result. When BCDMA was added, the color was concentrated in a layer on the bottom of the test tube (Figure 4-12). The sequestration of the diazo complex was not as complete, even when an excess of BCDMA was added. The first process was chosen to be adapted to the PMD.
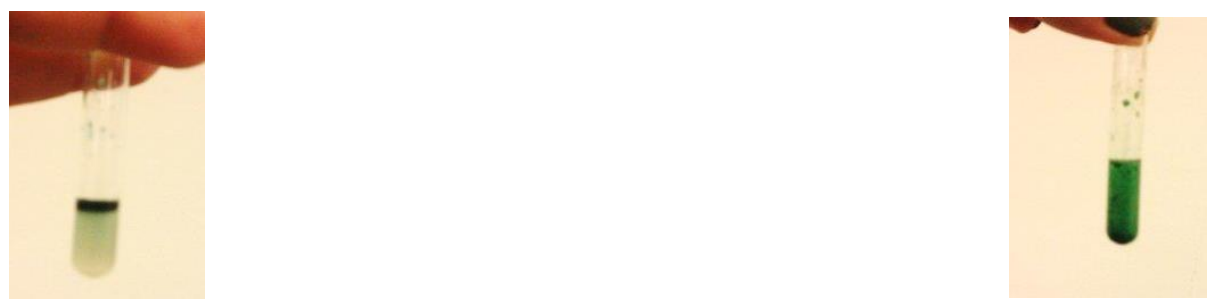

Figure 4-11. Addition of BCDMA, method [25]. Figure 4-12. Addition of BCDMA, method [24].

When transferring the test from [25] to the device, the solid reagents were rubbed into the chromatography paper. In the sample lane, the DCI and $\mathrm{NaOH}$ solution were layered between bands of trehalose to try to confine them on the test strip, slow their migration, and allow longer interaction times. Approximately 25 microliters of BCDMA solution was spotted in the top of the test loop to concentrate the color. Because of the number of reagents used and the small size of the test loop, the reagents bled together and the test chip did not function as designed. This test was sidelined in favor of Nessler's reagent, potassium tetraiodomercurate (La-Mar-Ka, Inc.; Baton Rouge, LA, USA) because a 
completed test chip was needed. The reaction product is a deep reddish-brown and the mechanism for the reaction between Nessler's reagent and the ammonium ion is outlined in Figure 4-13.

\section{$2 \mathrm{~K}_{2} \mathrm{HgI}_{4}+2 \mathrm{NH}_{3} \rightarrow \mathrm{NH}_{2} \mathrm{Hg}_{2} \mathrm{I}_{3}+\mathrm{NH}_{4} \mathrm{I}+4 \mathrm{KI}$}

Figure 4-13. Reaction mechanism for the formation of the reddish-brown color associated with Nessler's test for the ammonium ion.

The boric acid/DCI/1-naphthol test should be pursued because it provides a strong color change and could work with better reagent control, such as the use of an inkjet-type printer to "print" the reagents in finer patterns, and trehalose bands similarly printed in tight bands that do not overlap.

After further work a PMD for high/organic explosives was developed and the urea nitrate and peroxide tests were moved to this second PMD so all tests on this chip use the same solvent, 1:1 acetone and DI water. In the final iteration, chlorate and perchlorate tests were added to the three remaining tests, making this PMD a test for inorganic improvised or homemade explosive components. The solvent for all five ions is deionized water.

\section{Chlorate ion}

To detect chlorate, a saturated solution of aniline sulfate (Fisher Scientific; Fair Lawn, NJ, USA) in DI water was spotted at the midpoint of the sample lane and 50\% sulfuric acid (Fisher Scientific; Fair Lawn, NJ, USA) was spotted at the top of the sample lane (Figure 4-14).. 


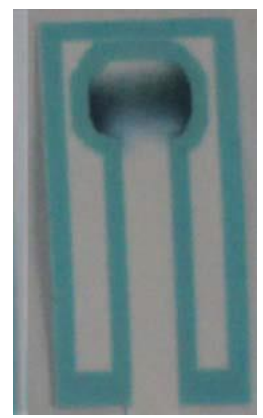

Figure 4-14. Positive test result for chlorate ion using aniline sulfate.

The reaction mechanism is outlined in Figure 4-15 below.

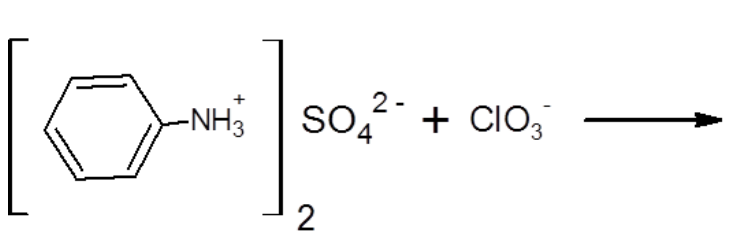

Aniline sulfate

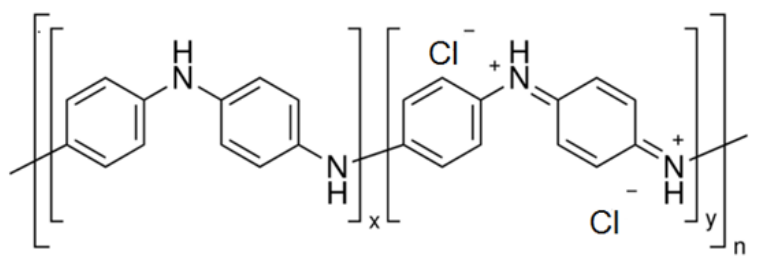

Polyaniline (emeraldine salt)

Figure 4-15. Color formation mechanism for the chlorate ion test.

\section{Perchlorate ion}

For perchlorate ion, a $0.05 \%$ aqueous methylene blue solution (Fisher Scientific; Fair Lawn, NJ, USA) was spotted at the top of the sample lane (Figure 4-16).

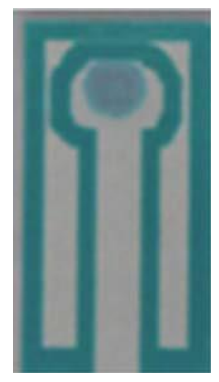

Figure 4-16. Positive test result for perchlorate ion using methylene blue.

The reaction mechanism is outlined in Figure 4-17 below. 


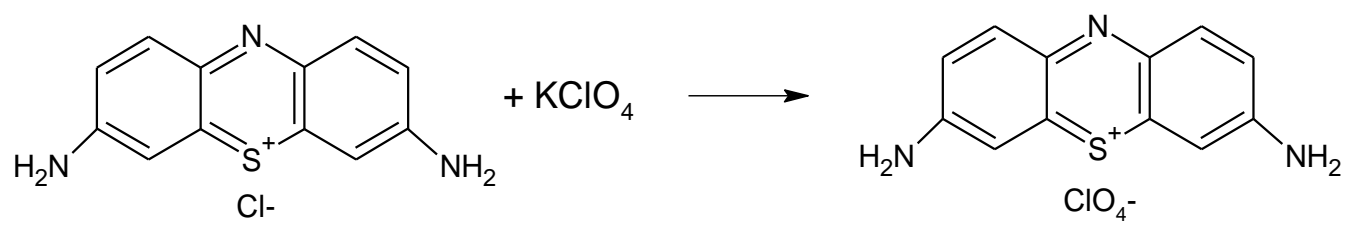

Figure 4-17. Color formation mechanism for the perchlorate ion test.

\section{F. Performance Test of Inorganic Paper Microfluidic Device: Proof of Concept}

\section{Background}

For the first performance test of the PMD, a set of 20 inorganic PMDs with nitrate ion, perchlorate ion, urea nitrate, chlorate ion, and nitrite ion tests were produced and used to test soil samples for the presence of inorganic explosive components. When present, the inorganic explosives in the soil samples were confirmed using FT-IR, X-ray powder diffraction, and microcrystalline tests.

This iteration of the device was used with the following test placements:

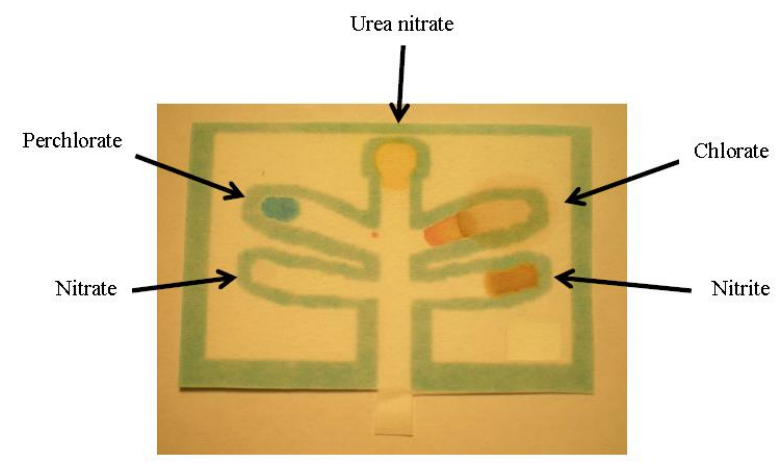

Figure 4-18. PMD developed for initial performance test.

After instrumental analysis, a portion of each soil sample was extracted and the extract tested using the paper microfluidic device. 


\section{Sample Preparation}

2 grams of soil were put in a test tube with approximately $2 \mathrm{ml}$ of the appropriate solvent. Each tube was vortexed for 30 seconds and spun down in a centrifuge for 60 seconds.

The extract was transferred to a $2 \mathrm{ml}$ vial, concentrated by evaporation to roughly $1 \mathrm{ml}$ as measured using graduations on the side of the $2 \mathrm{ml}$ vial, and the test run immediately. Representative samples of each positive result are shown below. All positive results are catalogued in the Appendix.

The devices were grouped into five sets of four. For each set, four different soil samples were extracted and the liquid extract tested using a PMD. Photo results for set \#1-1 through 1-4 were lost because of an SD card failure.

\begin{tabular}{|c|c|c|}
\hline Test ID & PMD results & $\begin{array}{l}\text { Instrumental } \\
\text { results }\end{array}$ \\
\hline 2-1 (negative control) & Negative & Negative \\
\hline $2-2$ & $\mathrm{ClO}_{3^{-}}$ & $\mathrm{KClO}_{3}$ \\
\hline $2-3$ & $\mathrm{ClO}_{3-}$ & $\mathrm{KClO}_{3}$ \\
\hline $2-4$ & Negative & $\mathrm{KClO}_{3}$ \\
\hline 3-1 (negative control) & Negative & Negative \\
\hline $3-2$ & $\begin{array}{l}\text { Initially negative, } \\
\text { slow } \mathrm{ClO}_{3^{-}}\end{array}$ & Negative \\
\hline $3-3$ & $\mathrm{ClO}_{3^{-}}$ & $\mathrm{KClO}_{3}$ \\
\hline $3-4$ & Negative & Negative \\
\hline 4-1 (negative control) & Negative & Negative \\
\hline $4-2$ & Urea nitrate & Urea nitrate \\
\hline $4-3$ & Negative & $\mathrm{NH}_{4} \mathrm{NO}_{3}$ \\
\hline $4-4$ & $\mathrm{NO}_{3^{-}}$ & $\mathrm{NH}_{4} \mathrm{NO}_{3}$ \\
\hline 5-1 (negative control) & Negative & Negative \\
\hline $5-2$ & Negative & $\mathrm{NH}_{4} \mathrm{NO}_{3}$ \\
\hline $5-3$ & Negative & Negative \\
\hline $5-4$ & $\mathrm{NO}_{3^{-}}$ & $\mathrm{NH}_{4} \mathrm{NO}_{3}$ \\
\hline
\end{tabular}

Table 4-1. Results of proof of concept tests from extracted soil samples. 


\section{G. Results}

All samples were first extracted and run by traditional analytical techniques: FTIR, X-ray powder diffraction (XRD), and microcrystalline tests. With the PMD, four samples were positive for chlorate ion, two were positive for nitrate ion, and one was positive for urea nitrate. For the remaining 8 out of 15 samples, the PMD gave a negative result. In three instances inorganic oxidizers were identified by laboratory instrumentation but the PMD did not give a corresponding positive result. This could be because of sample inhomogeneity (uneven distribution of the analyte) or the amount of analyte was below the threshold of the device. At this point, no detection limits were determined for each test.

It was also determined the dimensions of the device were too large to generate a "fast" result; the average time for the sample solution to be drawn to the top of the test lanes by capillary action and full color development to ensue was over 18 minutes. For all samples but 4-2, the samples were extracted with deionized water. Sample 4-2 (urea nitrate) was extracted with 1:1 methanol and acetone.

\section{Chlorate Ion Test}

The chlorate ion test reacted well and gave an unambiguous deep green positive result. However, the $50 \% \mathrm{H}_{2} \mathrm{SO}_{4}$ digested the paper substrate and the sample solution moved beyond the boundaries of the test lane, as observed in Figure 4-19. 


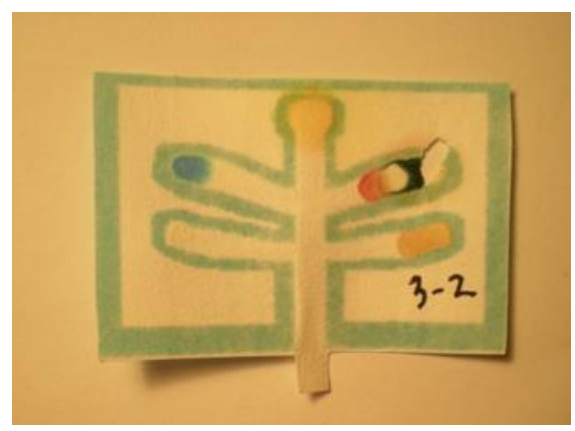

Figure 4-19. Representative positive chlorate test.

\section{Nitrate Ion Test}

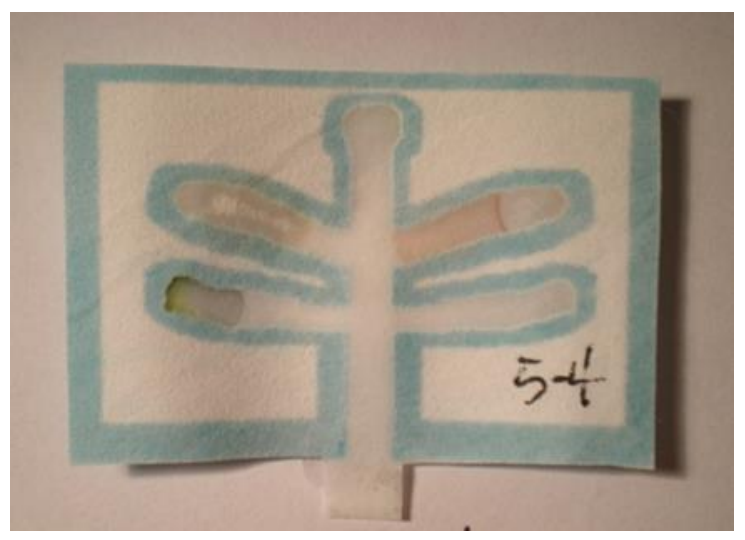

Figure 4-20. Representative positive nitrate test (weak).

This sample (Figure 4-20) contained a very low concentration of nitrate ion. Ammonium nitrate was detected in the sample by other analytical methods. No quantitation was performed.

\section{Urea Nitrate Test}

One sample gave a positive result for urea nitrate (Figure 4-21). There was some breakthrough of the sample solution (1:1 methanol and acetone) as shown by the red feathering outside the sample lane. The decision to use 1:1 methanol and acetone was 
made taking into account the results of the AN and UN solubility tests in Chapter 3 and the previous attempts to keep the color in the wax ink on the PMDs from mobilizing in pure acetone.

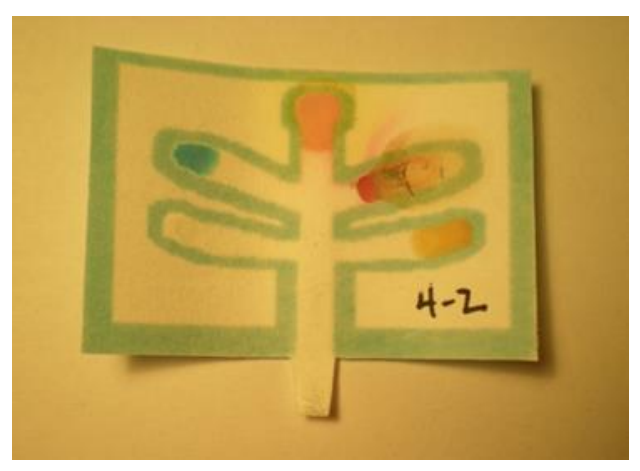

Figure 4-21. Positive urea nitrate test.

\section{H. Conclusions}

A five lane paper microfluidic device was developed for the analysis of unknown suspected explosive materials. The device is able to identify multiple components of inorganic explosives such as chlorate, perchlorate, nitrate, and nitrite, using deionized water or methanol as the solvent. Urea nitrate was detected using 1:1 acetone and methanol. In later iterations of the device, limits of detection ranged from $0.39-19.8 \mathrm{mg}$ of explosive compound [31], making the devices well suited for the identification of unknown powders recovered from improvised explosive manufacturing sites. Total analysis time of the revamped PMD was 5 minutes with very few steps needed to process the devices.

Compared to on-site detection techniques for explosive compound identification used today, these newly designed PMDs are simpler, smaller, and easily portable. They 
facilitate the identification of combinations of explosive compounds by permitting simultaneous multiplex testing. Therefore, these PMDs will provide law enforcement and military personnel with inexpensive and portable chemical tests for rapid determination of suspected explosive samples.

This project outlines the construction of a paper microfluidic device for the detection of explosive compounds and ions associated with explosive compounds. A successful initial test of that device was performed on soil samples containing some of the compounds of interest. While certain problems, such as the stability of the paper medium when concentrated acid is applied, must be addressed and overcome if this device is to be commercially viable this project demonstrated that these compounds can be detected by a paper microfluidic device. The tests were limited by the compounds available, which did not encompass all the tests on the test device, but the results obtained were correct and show proof of concept.

\section{References}

[1]. Hargadon, K., et al. J. Chrom. 1992; 602 (1-2), 241.

[2]. Dicinoski, G., et al. Forensic identification of inorganic explosives by ion chromatography. Anal. Lett. 2006, 39 (4); 639-657

[3]. McCord B et al. Forensic analysis of explosives using ion chromatographic methods. J. Chrom. 1994; $288(1-2), 43$.

[4]. Zhao, X., Yinon, J., Rapid Commun. Mass Spectrom. 15 (2001) 1514.

[5]. Tamiri, T., Rapid Commun. Mass Spectrom. 2005, 2094.

[6]. De Perre $\mathrm{C}$ et al. Rapid and specific detection of urea nitrate and ammonium nitrate by electrospray ionization time-of-flight mass spectrometry using infusion with crown ethers. Rapid Commun. Mass Spectrom. 2012; 26, 154-162 
[7]. Carrilho E et al. Understanding wax printing: A simple micropatterning process for paper-based microfluidics. Anal. Chem. 2009, (81) 16; 7091 - 95.

[8]. Whitesides, G.M., Nature 442 (2006) 368.

[9] Lu, Y., et al. Electrophoresis 30 (2009) 1497.

[10] Lu, Y., et al. Anal. Chem. 82 (2010) 329.

[11] Dungchai, W., et al. Analyst 136 (2011) 77.

[12] Li, X., et al. Chem. Commun. 46 (2010) 988.

[13] Martinez, A.W., et al. Anal. Chem. 80 (2008) 3699.

[14] Martinez, A.W., et al. Angew. Chem. Int. Ed. 46 (2007) 1318.

[15] Bruzewicz, D., Reches, M., Whitesides, G.M., Anal. Chem. 80 (2008) 3387.

[16] Feigl, F., Spot tests: Inorganic and organic applications, Nordemann, New York, 1937.

[17] United Nations Drug Control Programme, Technical Services Branch. Rapid

Testing Methods of Drugs of Abuse: Manual for Use by Law Enforcement and Narcotics Laboratory Personnel; New York, 1994.

[18] Palazzo G., et al. Sensors and Actuators B 161 (2012) 366.

[19]Mistral Group, Explosives detection field test kits. Accessed via http://www.mistralgroup.com/sec_explosives.asp on 16 April 2012.

[20] Almog J., Klein A., Tamiri, T., Shloosh,Y., Abramovich-Bar, S., J Forensic Sci 50 (2005) 582.

[21] Xu, M., et al. ACS Appl. Mater. Interfaces 3 (2011) 642.

[22] Makarov, S.Z., Ladelnova, L.V., Otdelenie Khimicheskikh Nauk (translation) 6 (1961) 958 .

[23] Dungchai, W., et al., Analytica Chimica Acta 674 (2010) 227.

[24] Asaoka, S., et al., Talanta 72 (2007), 1100.

[25] Hori, T., et al., Talanta 81 (2010) 1467. 
[26] Niki, K., et al., Anal. Methods, 2 (2010) 678.

[27] Fu, E., et al., Microfluid. Nanofluid. 10 (2011) 29.

[28] Liu, H., Crooks, R.M., J. Am. Chem. Soc. 133 (2011) 17564.

[29] Lim, S.H., et al. Org. Lett. 10 (2008) 4405.

[30] Kadarmandalgi, S.G., J. Chem. Educ. $41(1964,41) 437$.

[31] Peters, K.L., Corbin, I., Kaufman, L.M., Zreibe, K., Blanes,L., McCord, B., Anal. Methods, 2015, 7, 63. 


\section{CHAPTER 5: Conclusions and Future Work}

\section{A. Separation and Identification of Organic Components of Smokeless Gunpowder by Capillary Electrochromatography}

\section{Conclusions}

While the organic and inorganic components of smokeless powders have been analyzed using many different techniques, this is the first report of a CEC-ESI-TOF-MS procedure used to identify the organic components of smokeless powder. A hexyl acrylate-based monolith was synthesized to effect the CEC separation, which was coupled first to UV detection. UV data showed the fourteen components in the standard mixture were all resolved satisfactorily. The components of the standard mixture were infused directly into the TOF-MS to optimize conditions, observe the adducts formed and determine the $\mathrm{m} / \mathrm{z}$ error, which ranged from -14.5 to $3.7 \mathrm{ppm}$. Figures of merit including linearity, detection limits, and repeatability for both UV and TOF-MS were determined by running the 14part standard mix.

Five commercial smokeless powders were prepared and analyzed using this method. The UV and MS results were complementary and allowed all five powders to be differentiated. The small injection volumes and minimal amount of sample required make this a promising forensic technique for small sample sizes.

\section{Future work}

CEC-ESI-TOF-MS has shown itself to be a sensitive and selective technique for the analysis of organic additives of smokeless powder. A logical next step would be to continue to analyze more intact commercial smokeless powders to create a database. Since smokeless powders are manufactured all over the world, a database cataloguing the 
organic components present in smokeless powders from other countries could be of use to law enforcement and, potentially, the US military's forensic intelligence efforts. Another avenue for further work is the effect of ambient humidity on the synthesis of the hexyl acrylate-based monolith. It was noted that on days of especially high ambient humidity the monolith mixture took on a cloudy aspect and the finished monolith either had gaps in the polymer after conditioning or did not adhere to the walls of the capillary and was pushed out by the nitrogen gas when the vials were pressurized. Pretreating the walls of the capillary with an acetone rinse and then conditioning with ammonium hydroxide solution prior to introduction of the monolith mixture helped with adhesion to the capillary wall and made the monolith less likely to be pushed out under pressure.

\section{B. Detection of the Improvised Explosives Ammonium Nitrate and Urea Nitrate using Nonaqueous Solvents with Electrospray Ionization and MS/MS Detection}

\section{Conclusions}

In this paper a method for the determination of urea and ammonium nitrate by nonaqueous electrospray MS/MS is described. Examination of the mass spectral data permits the differentiation of true urea nitrate from samples containing mixtures of urea, a nitrate salt, and an acidic proton source. Even though both contain a peak at m/z 185 corresponding to the [urea nitrate $\left.+\mathrm{NO}_{3}\right]^{-}$cluster, the AN/urea mixture also shows peaks at $\mathrm{m} / \mathrm{z} 182$ and $\mathrm{m} / \mathrm{z} 242$ because of the presence of cluster ions containing urea that are not present in a mass spectrum of true urea nitrate. Additionally, the m/z 248 peak corresponding to [urea nitrate $\left.+\mathrm{HNO}_{3}+\mathrm{NO}_{3}\right]^{-}$will be substantially reduced in a mixed sample. 
Taking a simple ratio of the $\mathrm{m} / \mathrm{z} 248: 185$ peaks also aids in the determination of whether a sample contains urea nitrate. For true UN samples the ratio is greater than 1 and for mixtures, the ratio is approximately 0.2 . If the mixture does not have an acidic proton source present, the $\mathrm{m} / \mathrm{z} 185$ ion is not produced. Interfering compounds show a wide variety of influences on urea nitrate. Oils and fuels have minimal effects, while aqueous liquids and cleaning products have detrimental and complicating effects on the mass spectrum. Overall this procedure provides a rapid method for quickly screening for the presence of these improvised explosives on a variety of surfaces.

\section{Future Work}

One of the most challenging problems in the analysis of urea nitrate and ammonium nitrate is to find a nonaqueous solvent that will put both compounds into solution for analysis without dissociating urea nitrate into its constituents, urea and nitrate ion. With both compounds so common in the environment, it cannot be concluded that detection of the components proves the urea nitrate molecule was once present. Ionic liquids may offer a way to stabilize the compounds of interest

\section{Development of a Paper Microfluidic Device for the Detection of Improvised} Explosives

\section{Conclusions}

A five-lane PMD was developed for the analysis of unknown suspected explosive materials. It was designed to detect multiple components of inorganic explosives such as chlorate, perchlorate, nitrate, nitrite, and urea nitrate, using deionized water as the solvent for all analytes but urea nitrate, which required a 1:1 mixture of methanol and acetone. 
Total analysis time for these PMDs was 18 minutes, which made a redesign necessary for succeeding versions of the PMD. Compared to on-site detection techniques for explosive compound identification used today, these newly designed PMDs are simple, small, and easily portable. They have the potential to facilitate the identification of combinations of explosive compounds by permitting simultaneous multiplex testing. These PMDs could provide law enforcement and military personnel with inexpensive and portable chemical tests for rapid determination of suspected explosive samples.

\section{Future Work}

There is ample opportunity for future work on the selection and optimization of color tests for the PMD.

The effect of BCDMA on the fixing and concentration of color in the results area of the PMD sample loop has the potential to be exploited. This compound, if placed in a small enough area, could be useful to hold and concentrate the colored complex that forms as a result of these tests. It could be examined for use in every test chosen so far; p-DMAC, ATO, and the tests for nitrate and ammonium ions.

\section{a. Pending Color Tests}

Promising color tests left to evaluate include:

Phenylanthranilic acid/sulfuric acid for chlorates: this test was found on a pyrotechnics website [1] and states that nitrate, chloride, and most importantly perchlorate do not interfere with the test. 
Aluminum $\left(\mathrm{Al}^{3+}\right)$ : aluminon reagent (triammonium salt of aurintricarboxylic acid). When the ammonium salt is combined with $\mathrm{Al}^{3+}$ ions it forms the white precipitate $\mathrm{Al}(\mathrm{OH})_{3}$ which binds the aluminon, forming a red-orange precipitate.

Zinc: since zinc is used in the reduction of nitrate to nitrite in the Griess test, perhaps a reverse Griess test could be performed.

Sugar: most available tests are for reducing sugars, and sucrose (table sugar) is a nonreducing sugar. Musto [2] engineered an array of boronic acids to identify monosaccharides, disaccharides, and artificial sweeteners. Sixteen compounds were used for this array but since sucrose is the target of interest, the array could be narrowed down to be specific for sucrose.

\section{b. Construction of the Final PMD Chip}

To minimize sample volume needed, a better drawing program such as CorelDraw is needed to optimize the width of the channels and arrange the multiple tests leading from the central channel in a way that maximizes the number of tests than can be arrayed on a chip. Alternately, a three-dimensional design could be produced by printing a series of hydrophobic circles and lines which, when folded correctly, form a device with a threedimensional network of channels leading to multiple test reagent sites from one sample application point [4]. 


\section{References}

[1] Utah Pyrotechnics Association, Analysis of Perchlorates. Accessed via http://utahpyro.org/compositions/AnalysisofPerchlorates.pdf on 26 November 2013

[2] Musto, C.J., Suslick, K.S., Curr. Opin. Chem. Biol (2010) 14, 1.

[3] Liu H., Crooks, R.M., J. Am. Chem. Soc. (2011) 133, 17564. 
VI. Appendices, ESI-MS Data for UN Interferent Tests 

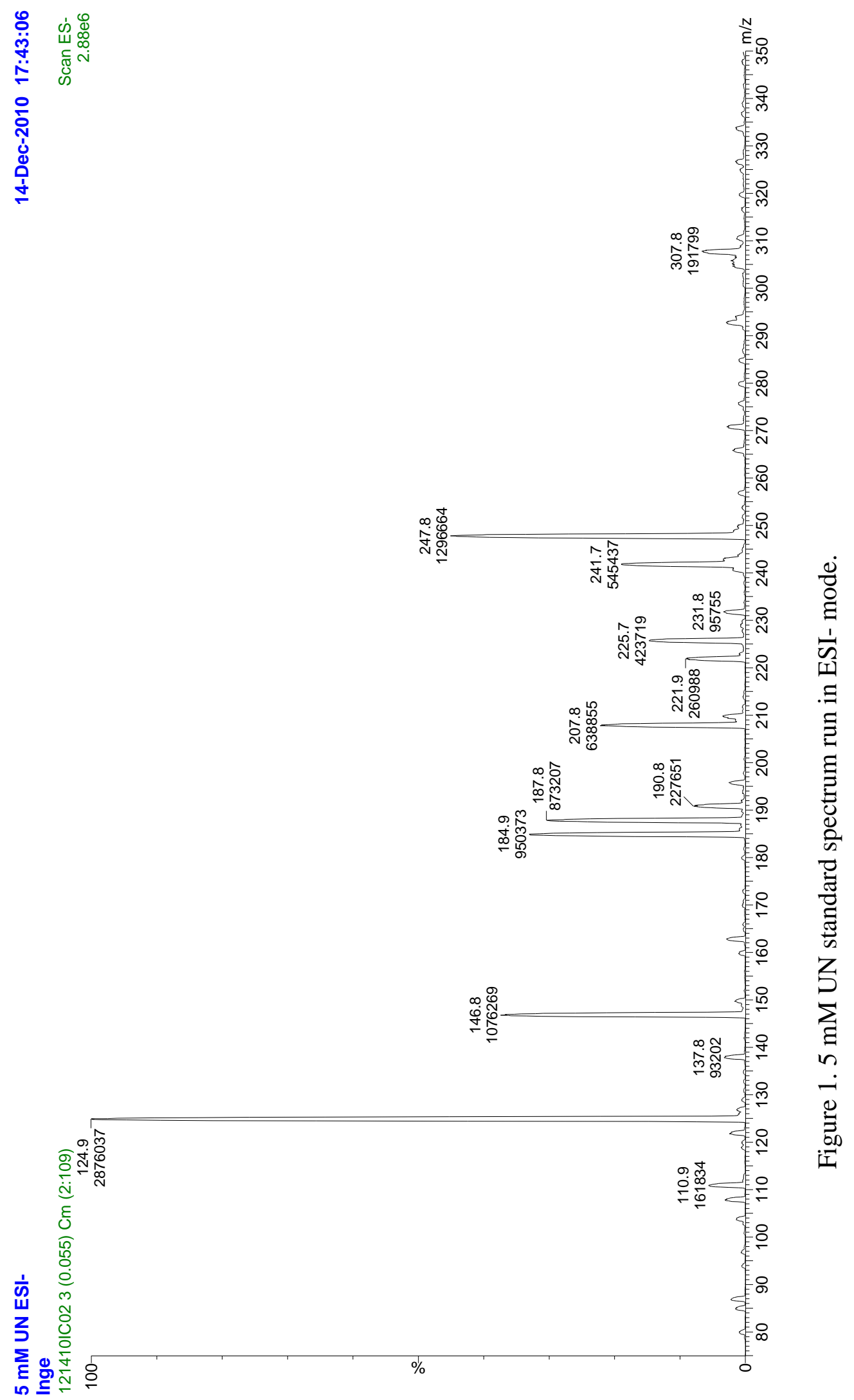

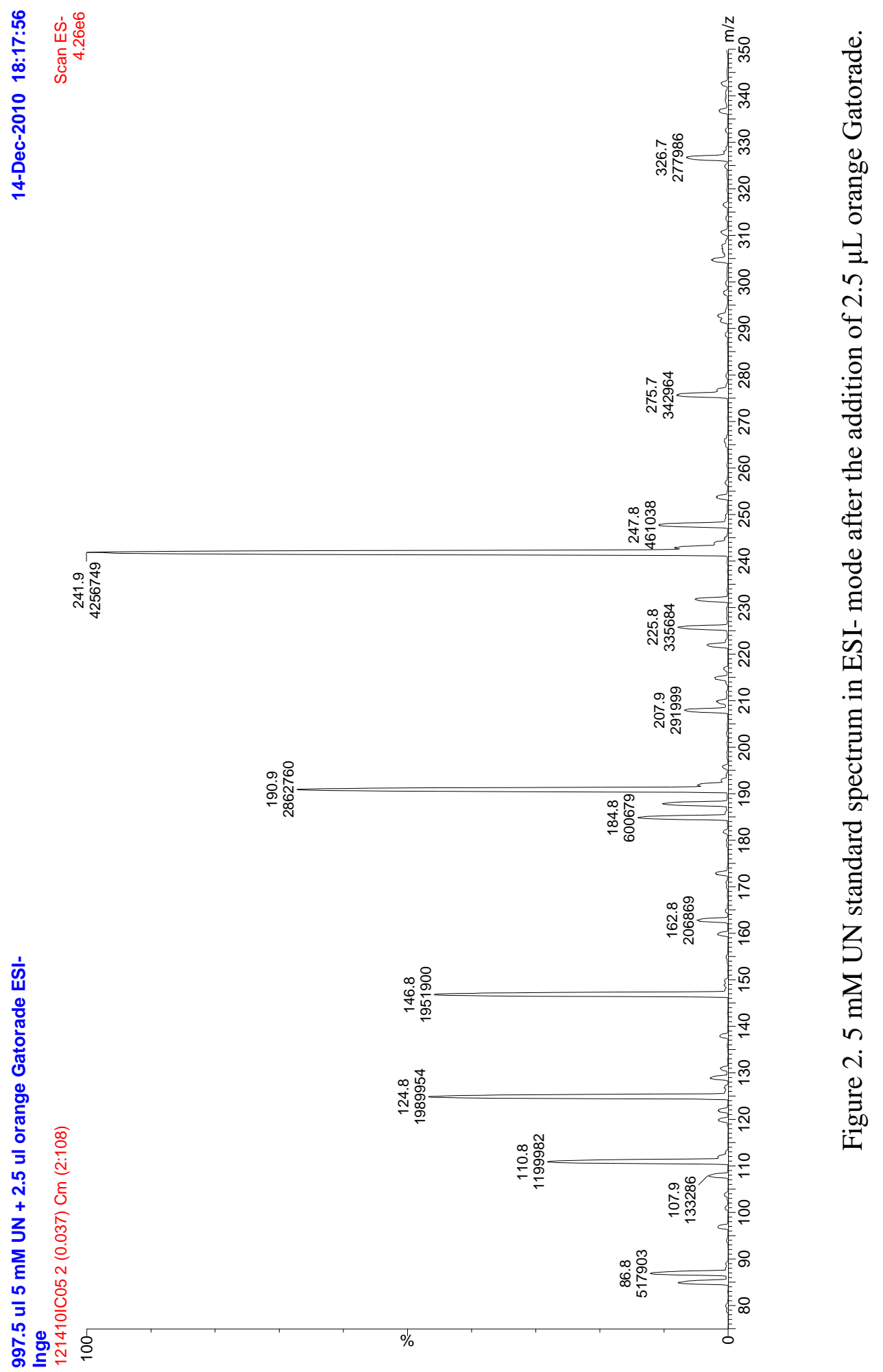

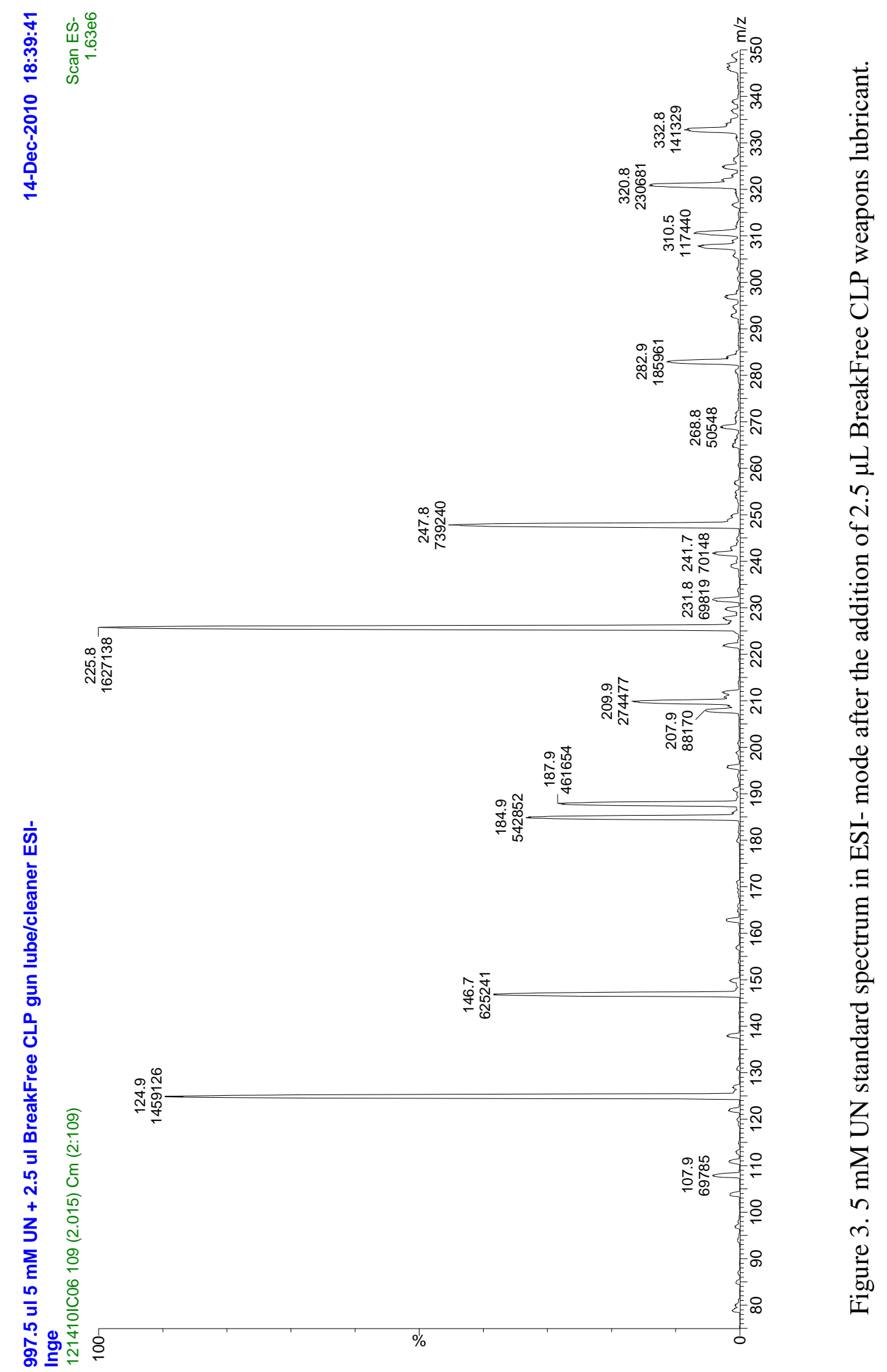

Z 

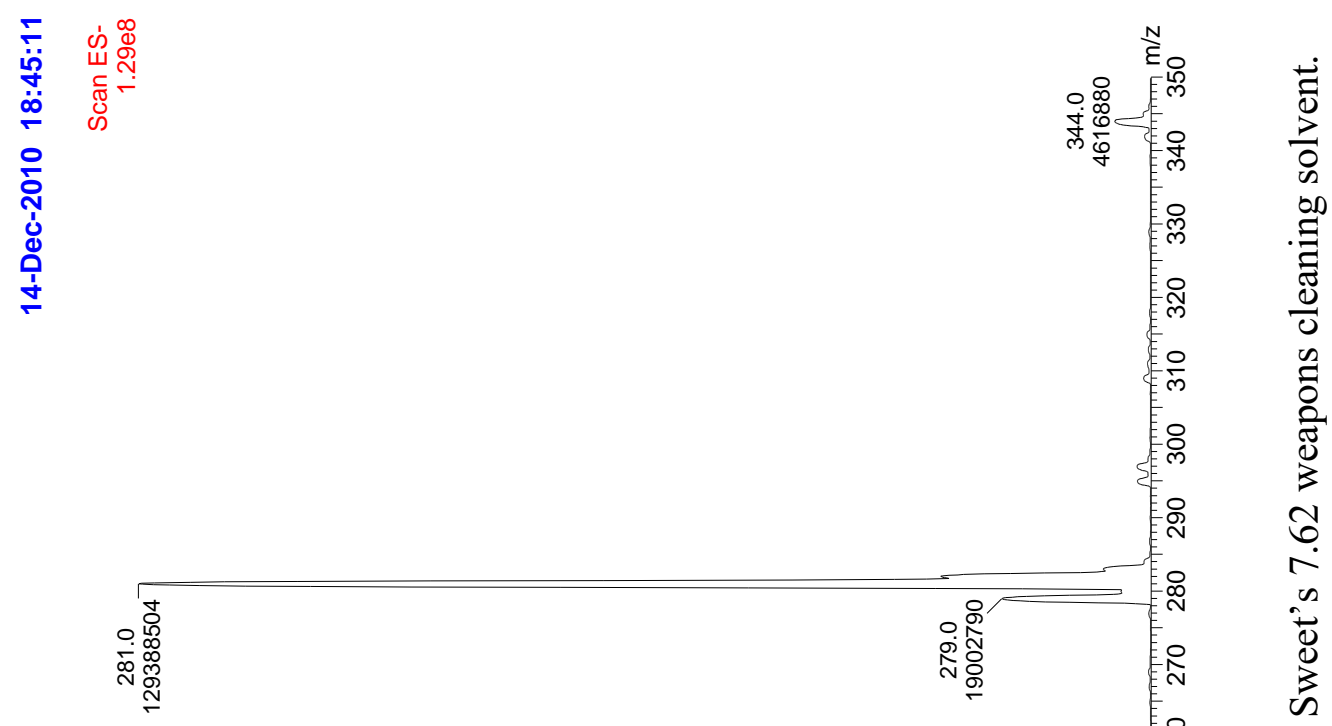

으

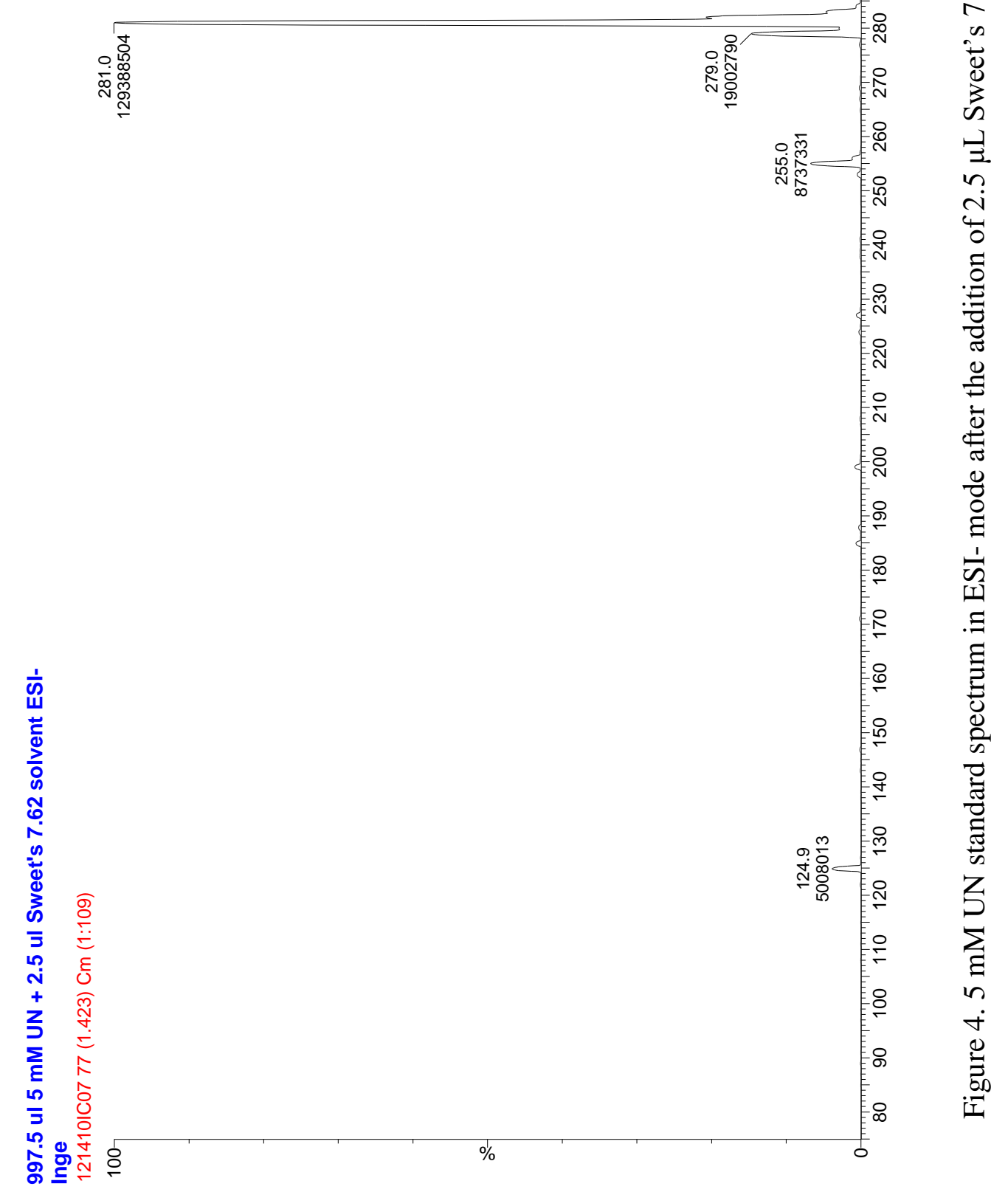



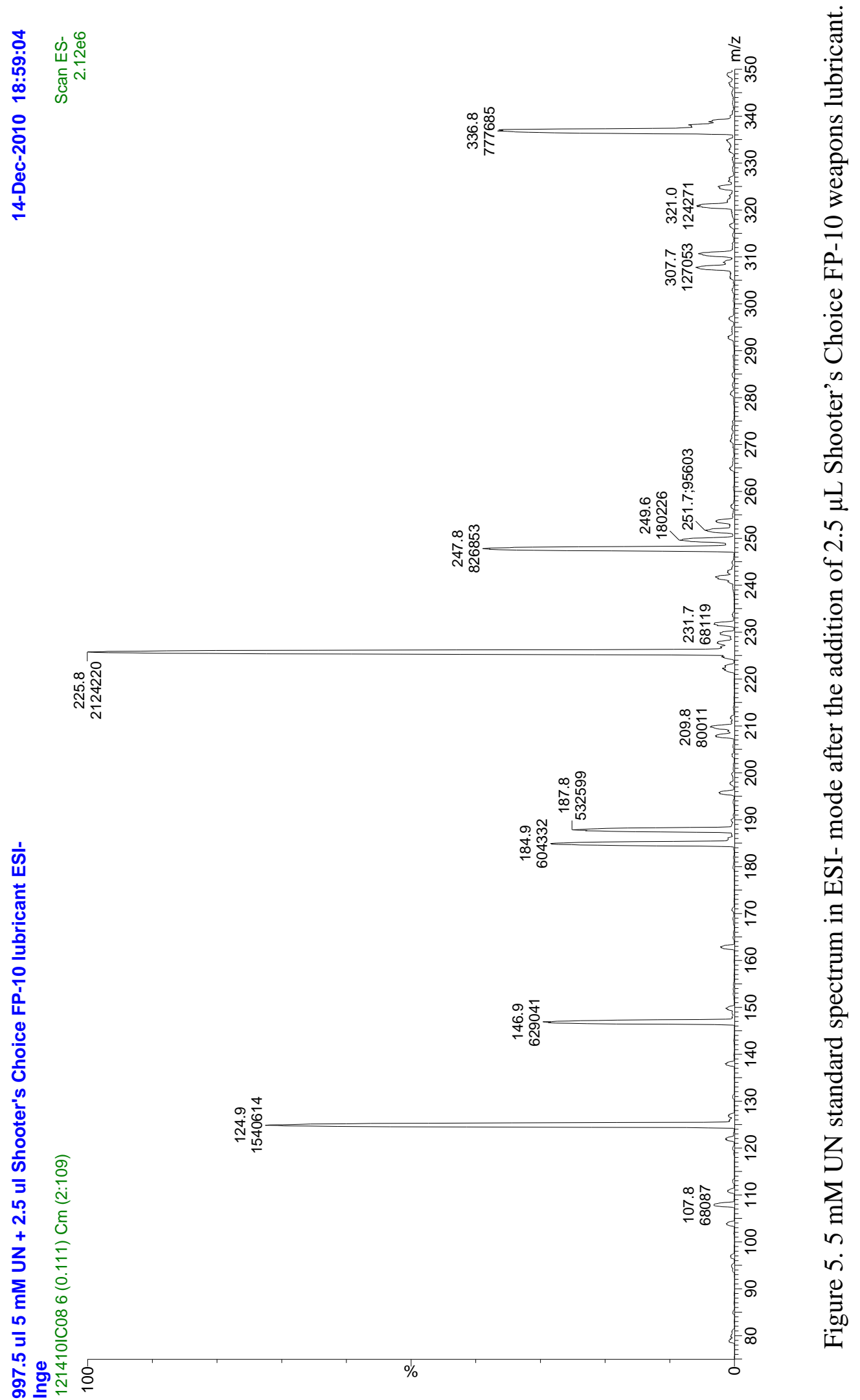

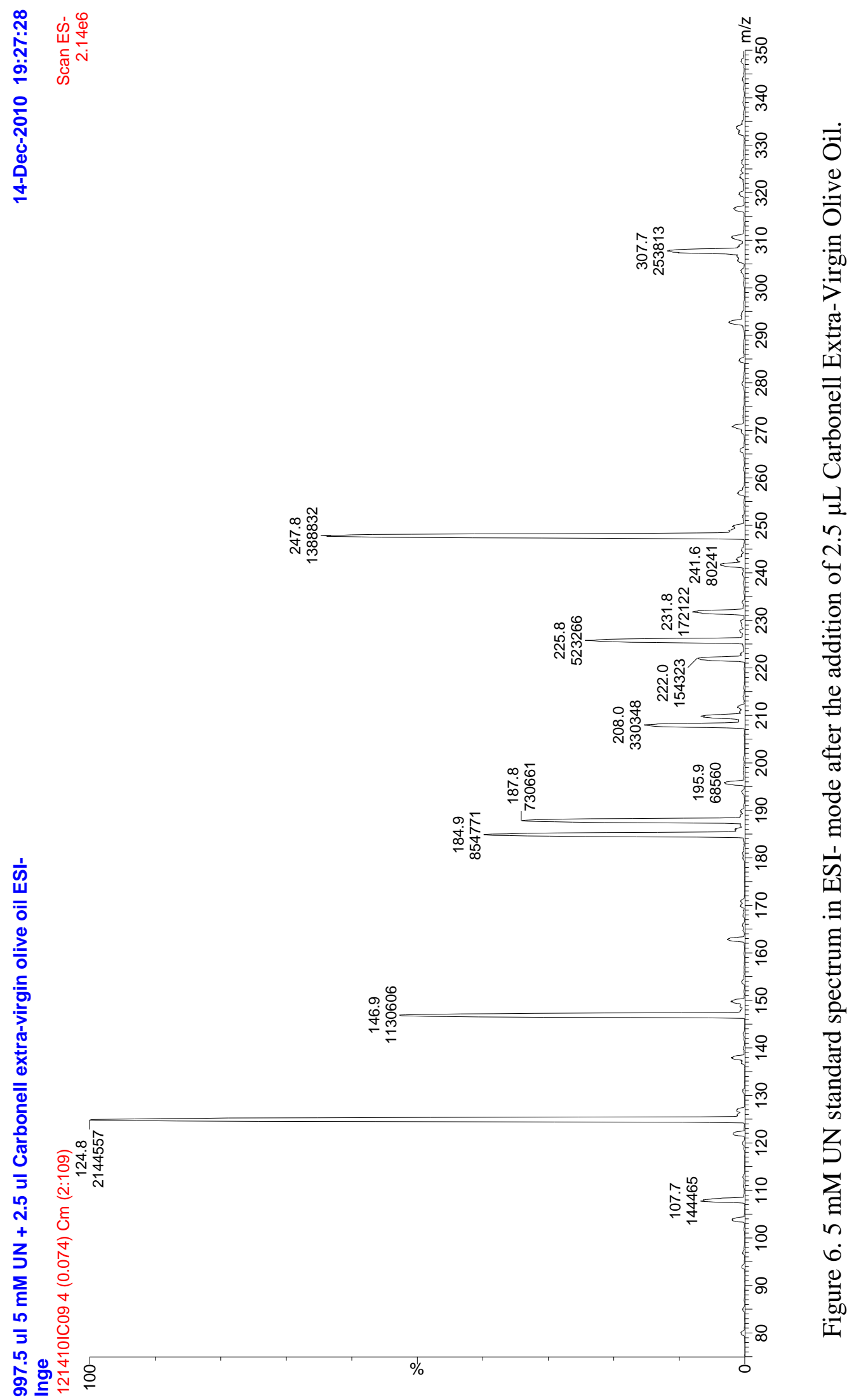

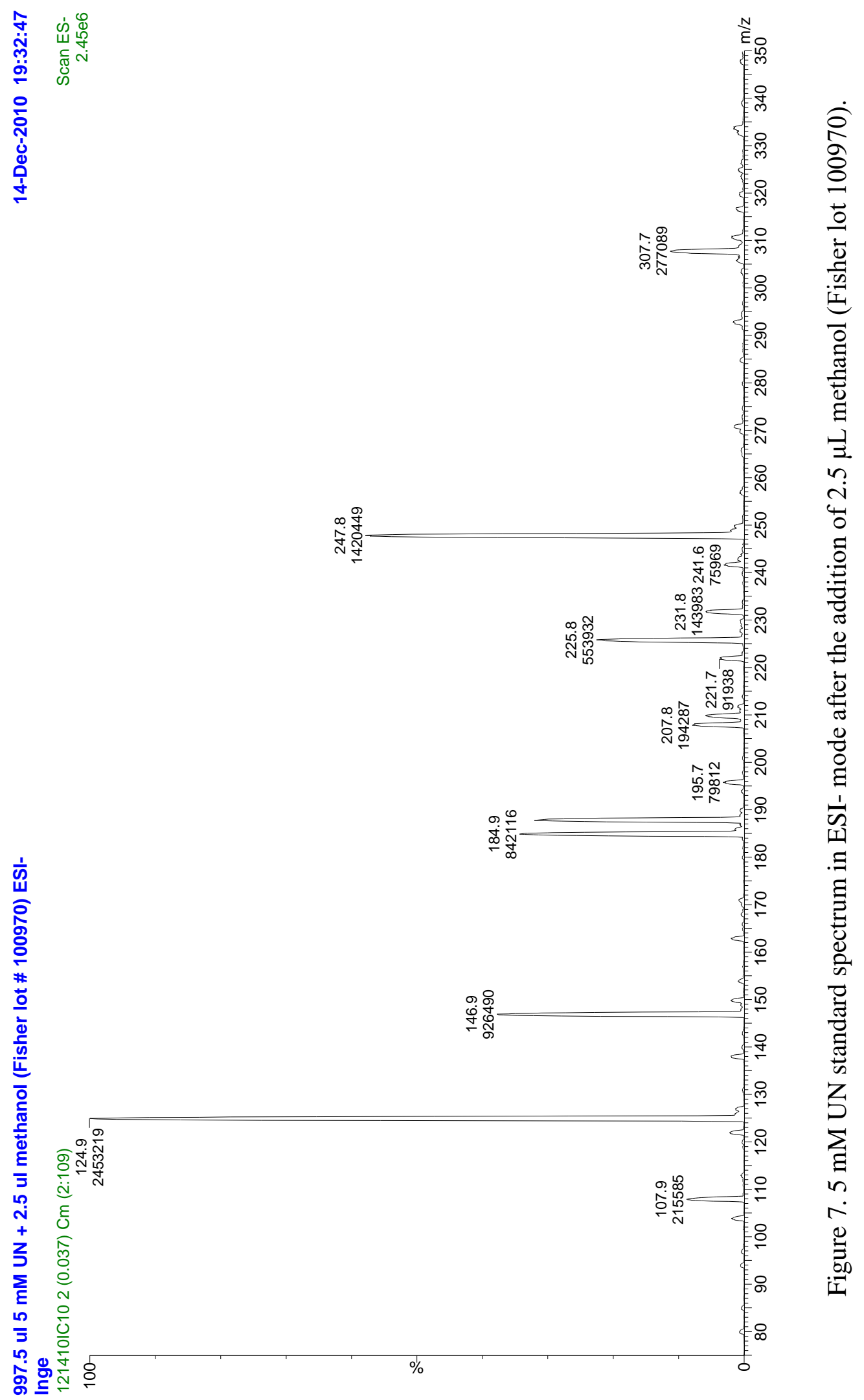

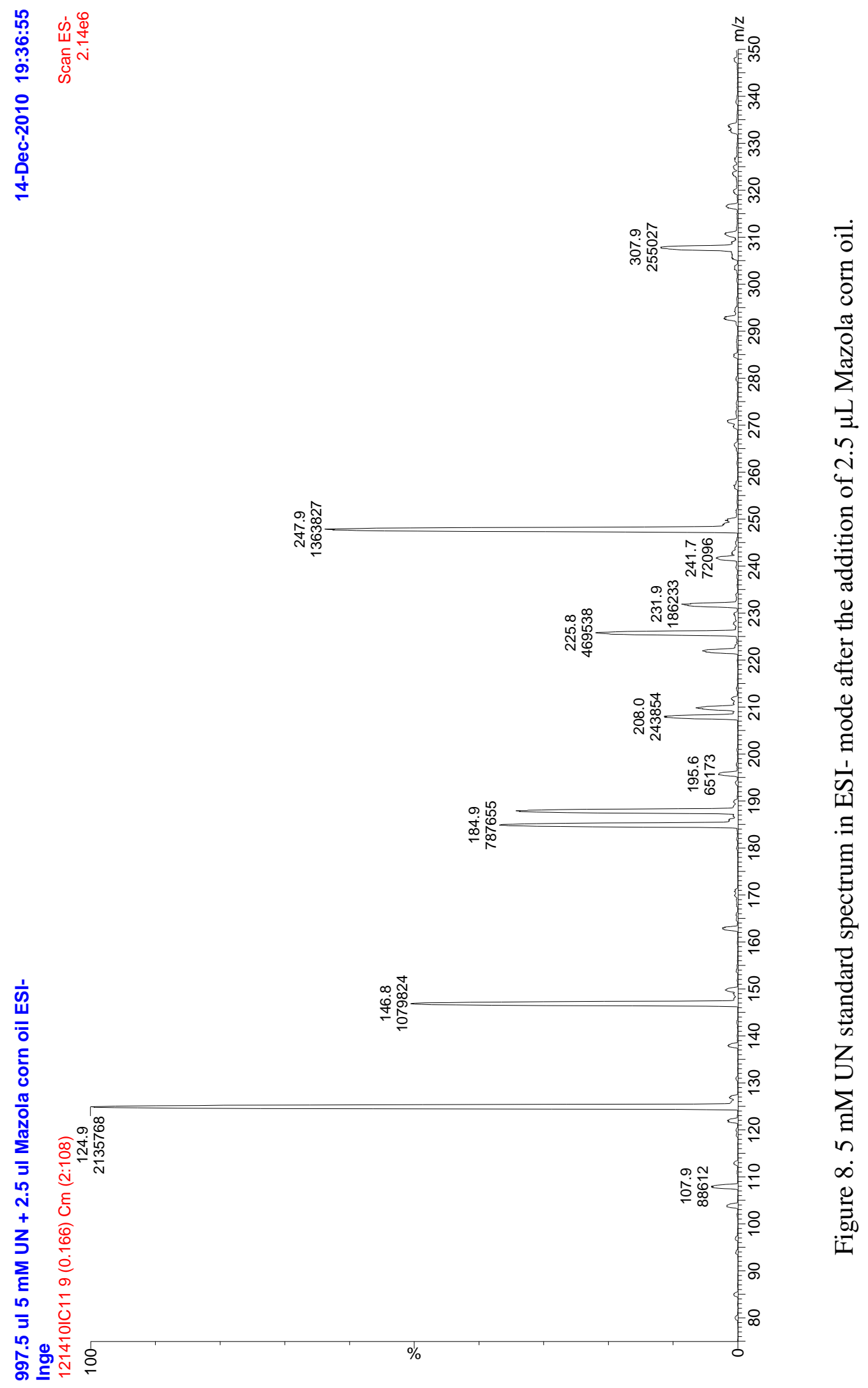

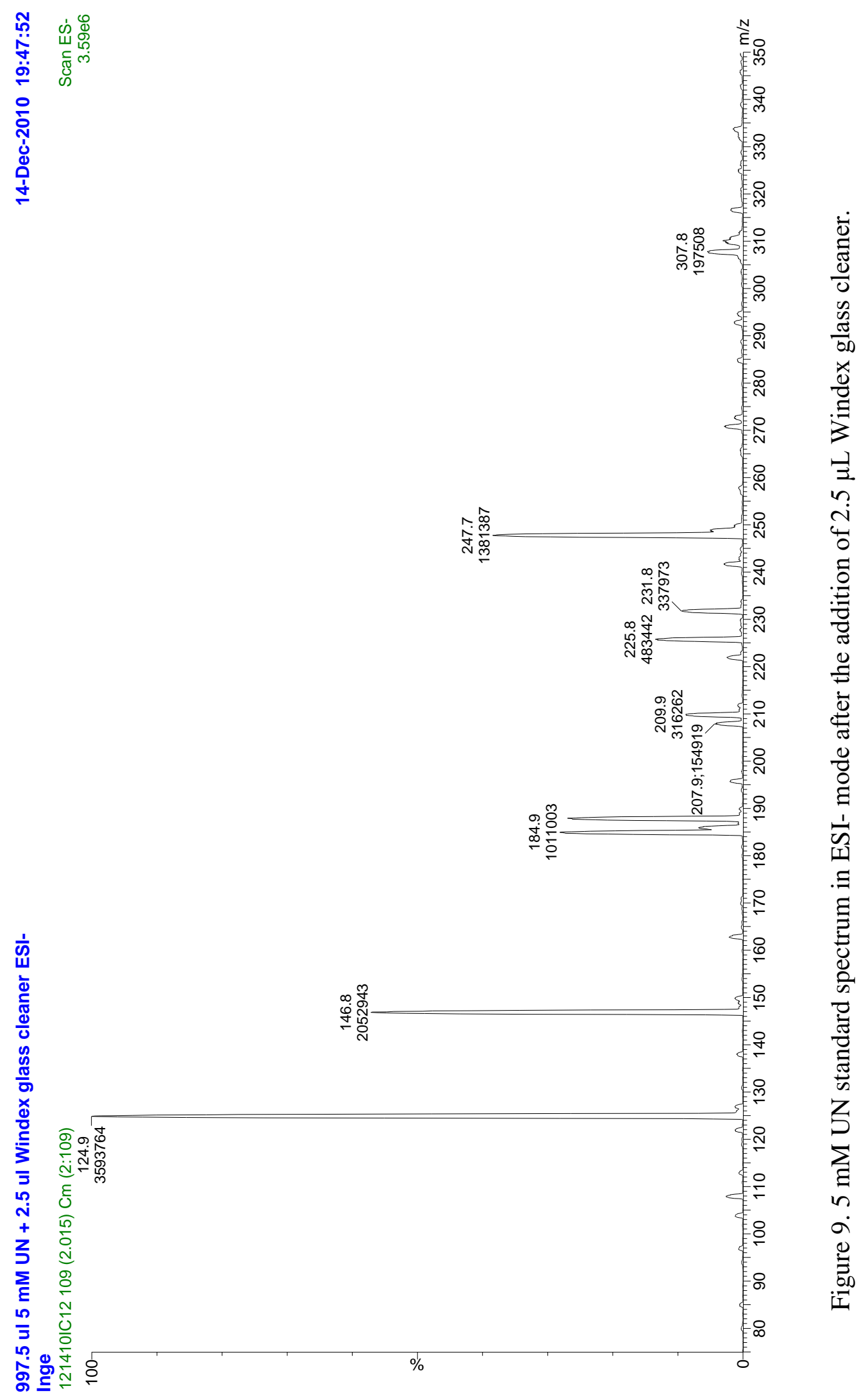

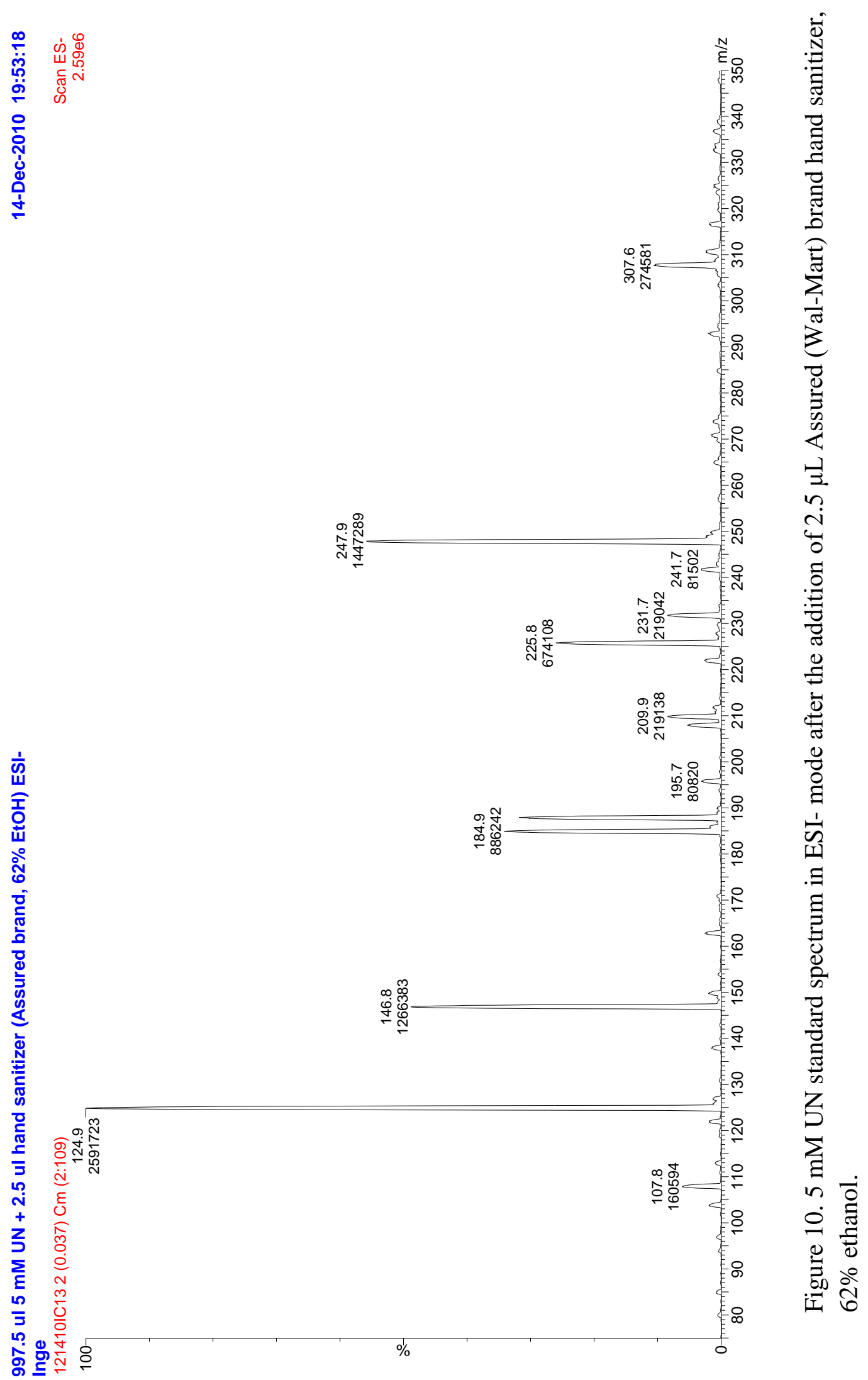

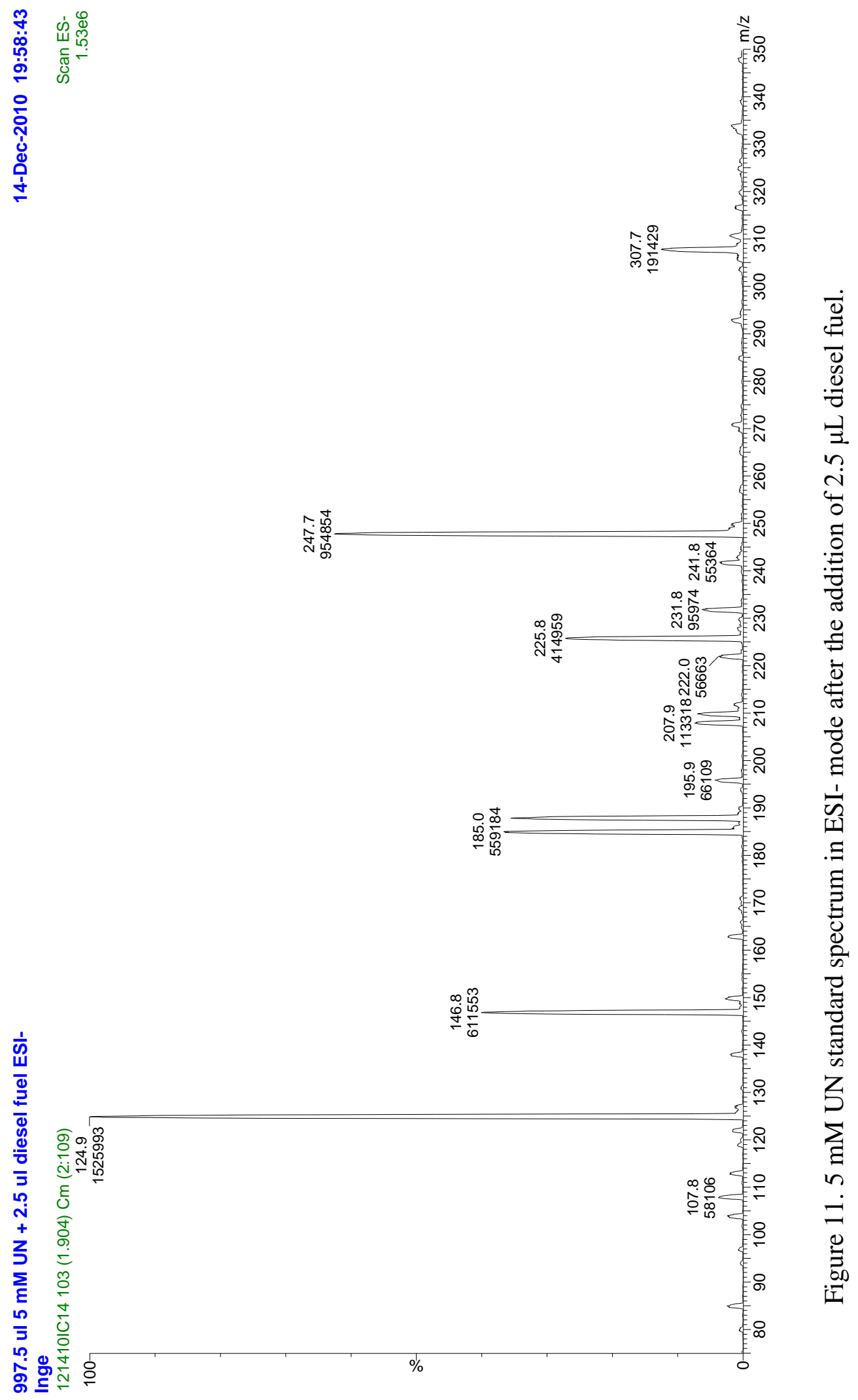

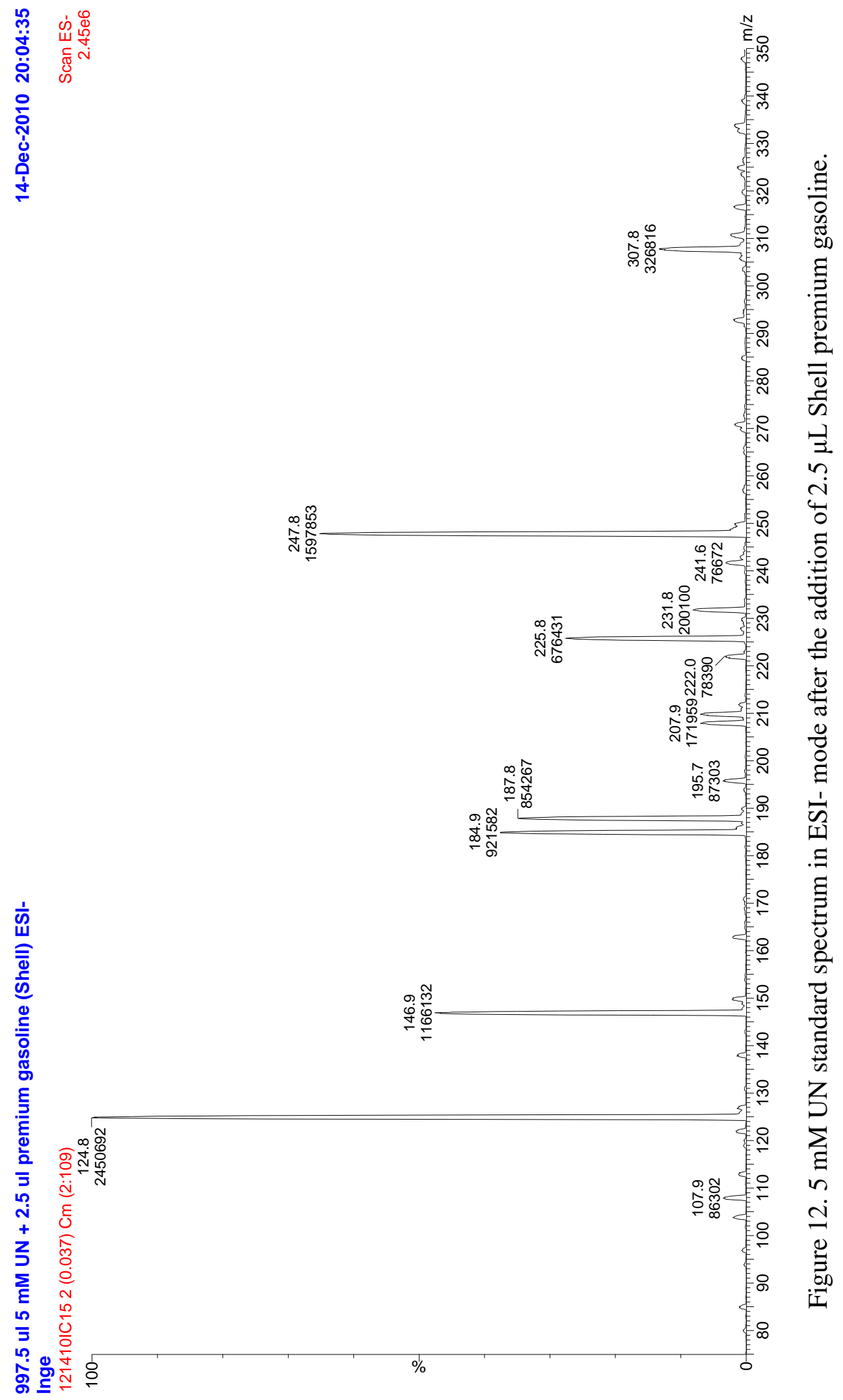

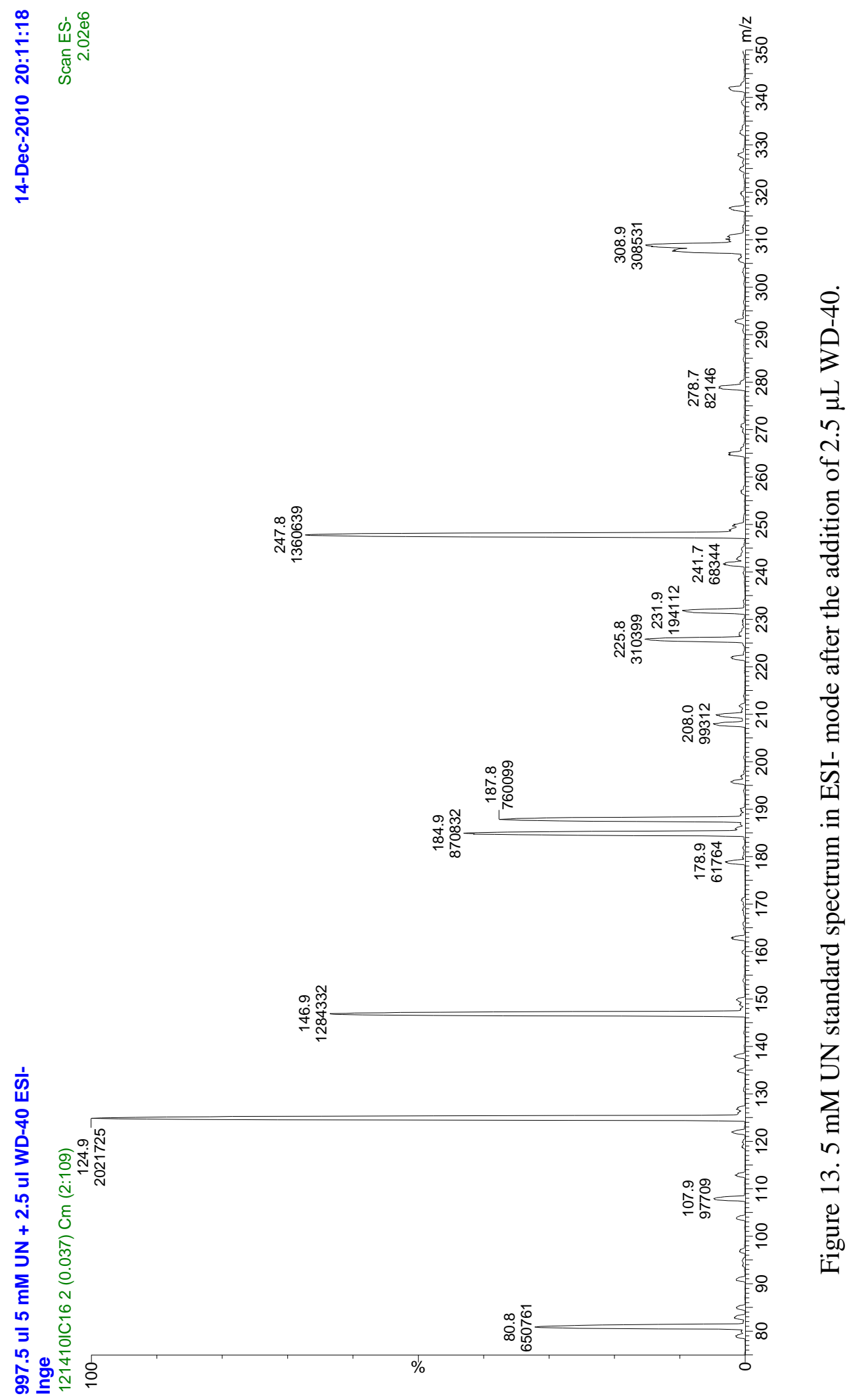
VII. Appendix B, Development of a Paper Microfluidic Device for the Detection of Improvised Explosives 

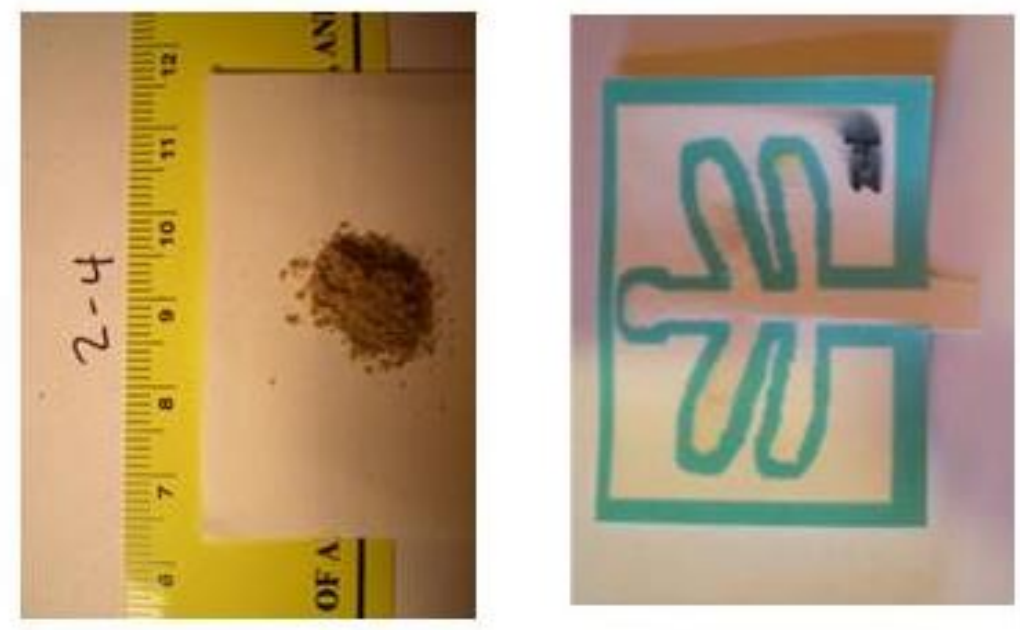

空

营
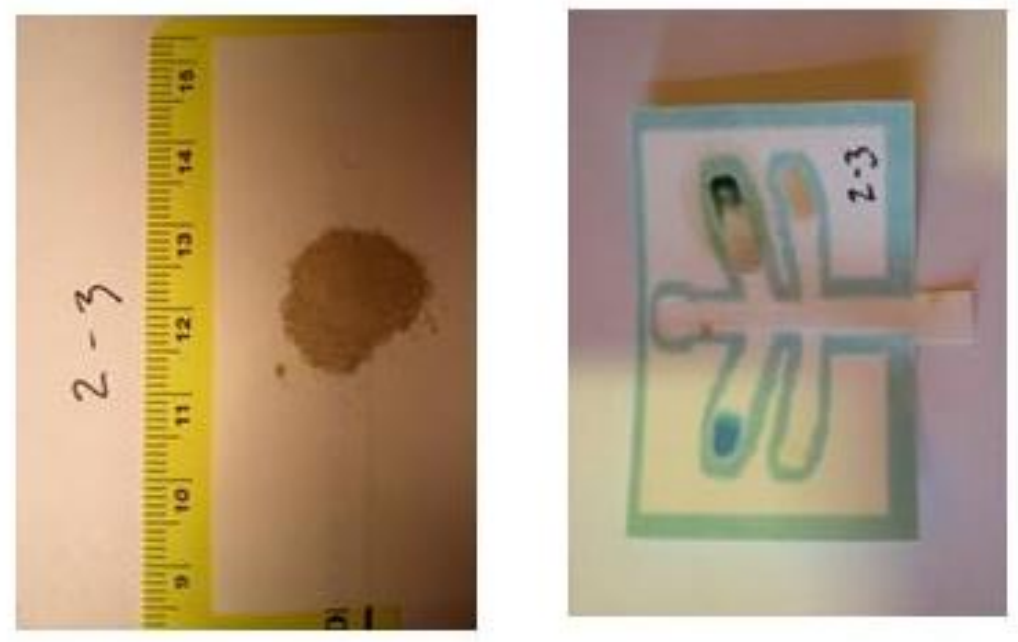

눔

葛

葛

迕

वे
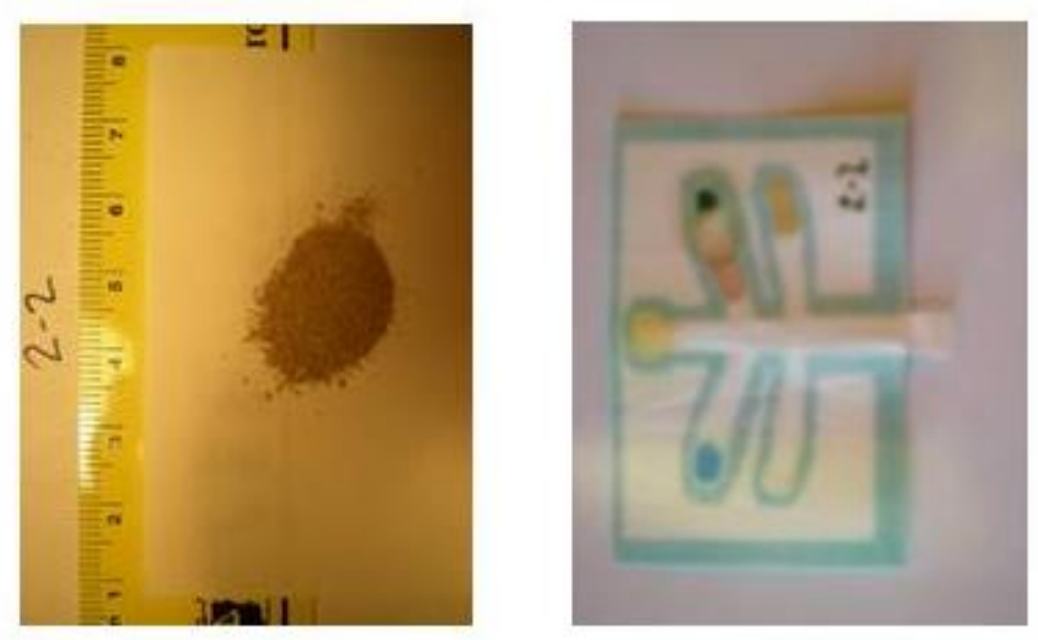

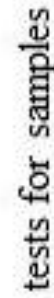

总 

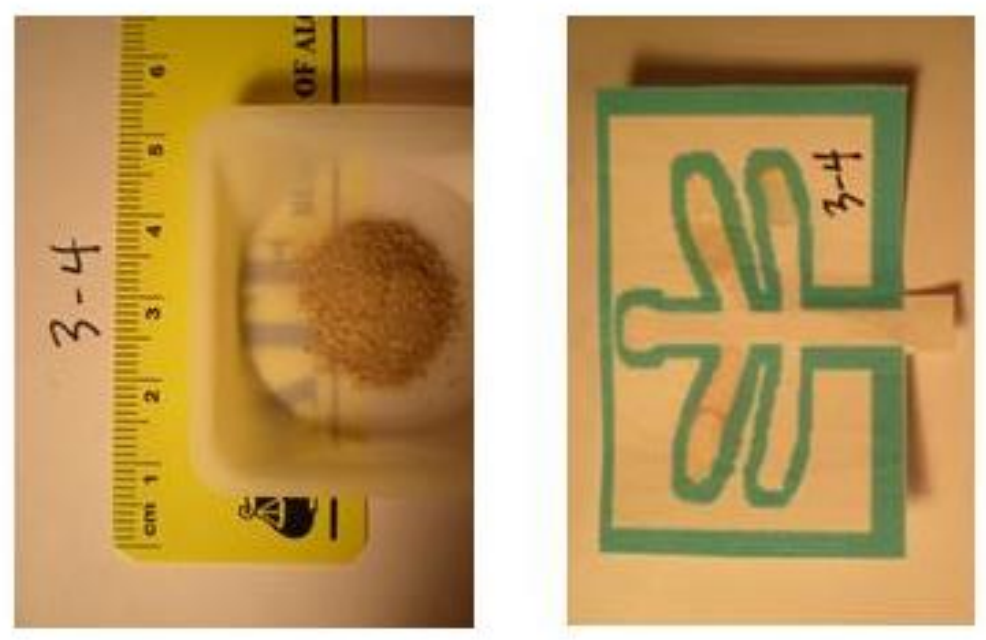

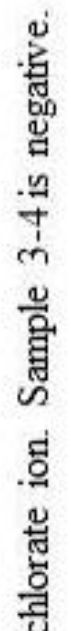
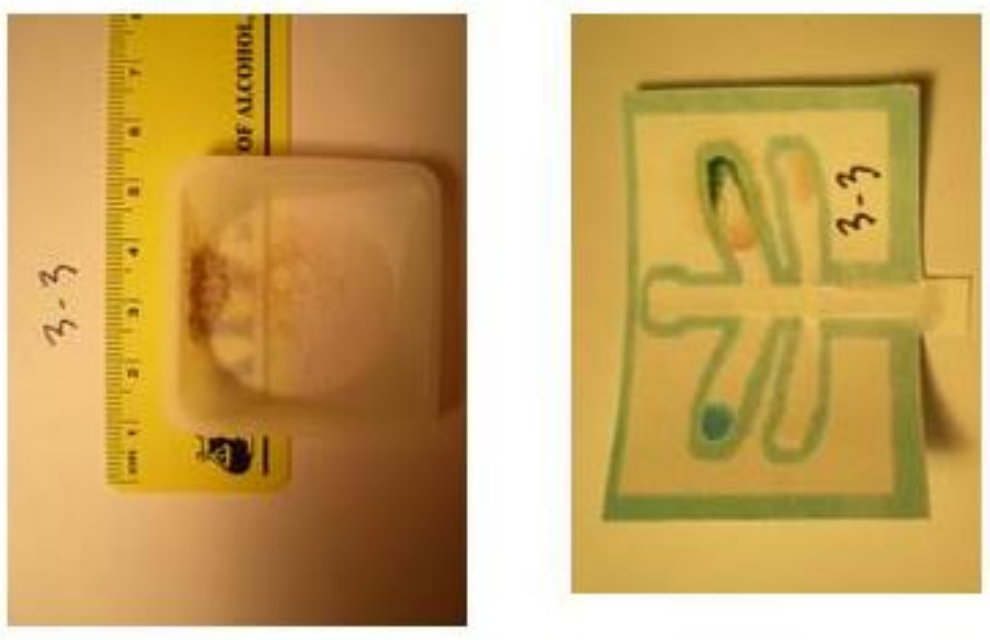

点
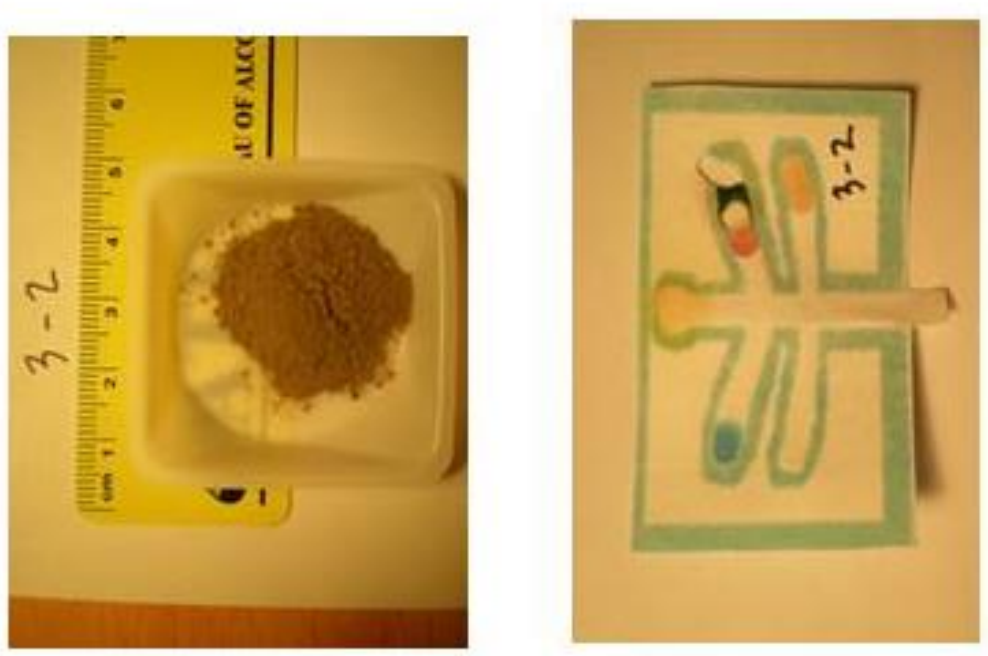

泀

ले

获

t5

离

旁 


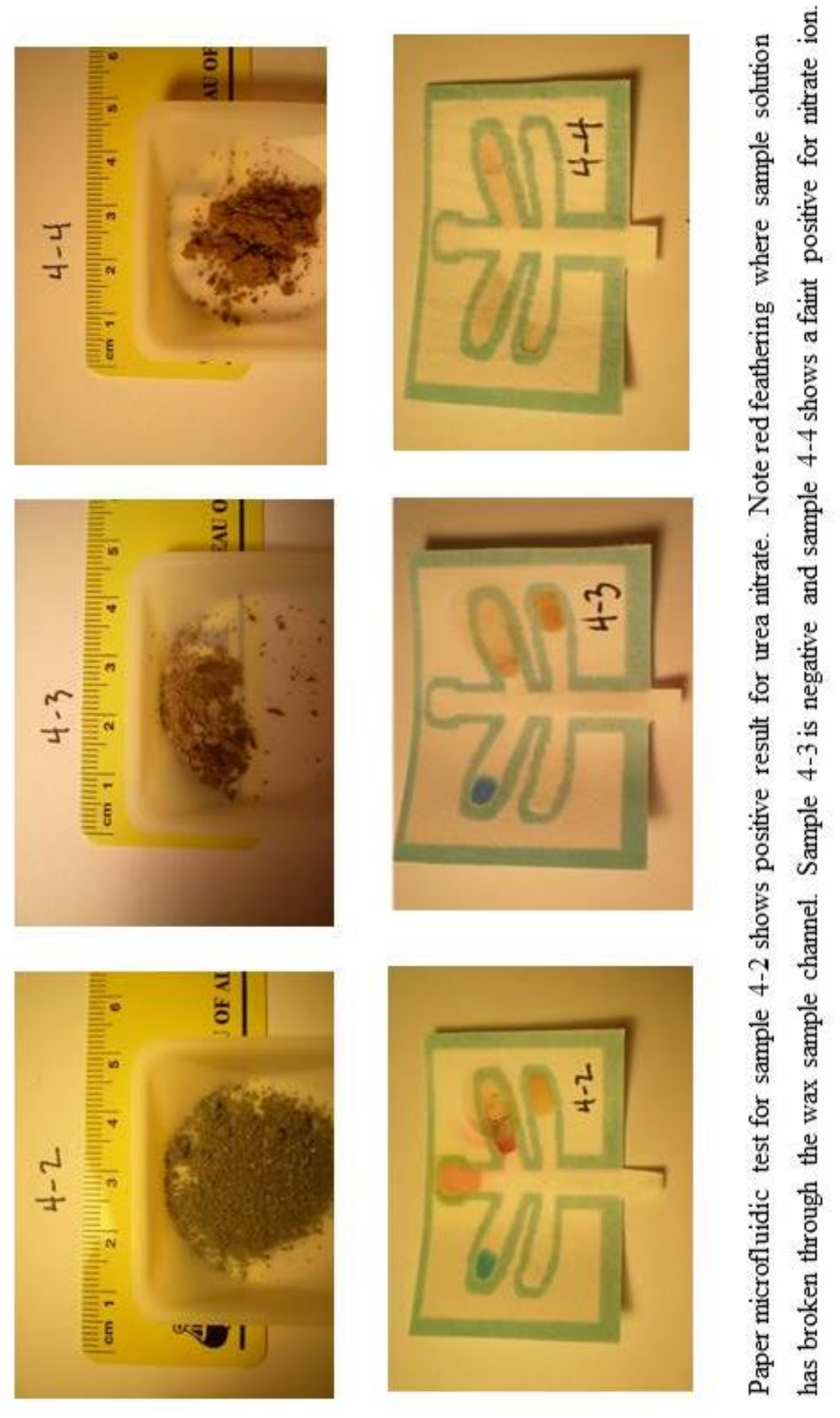



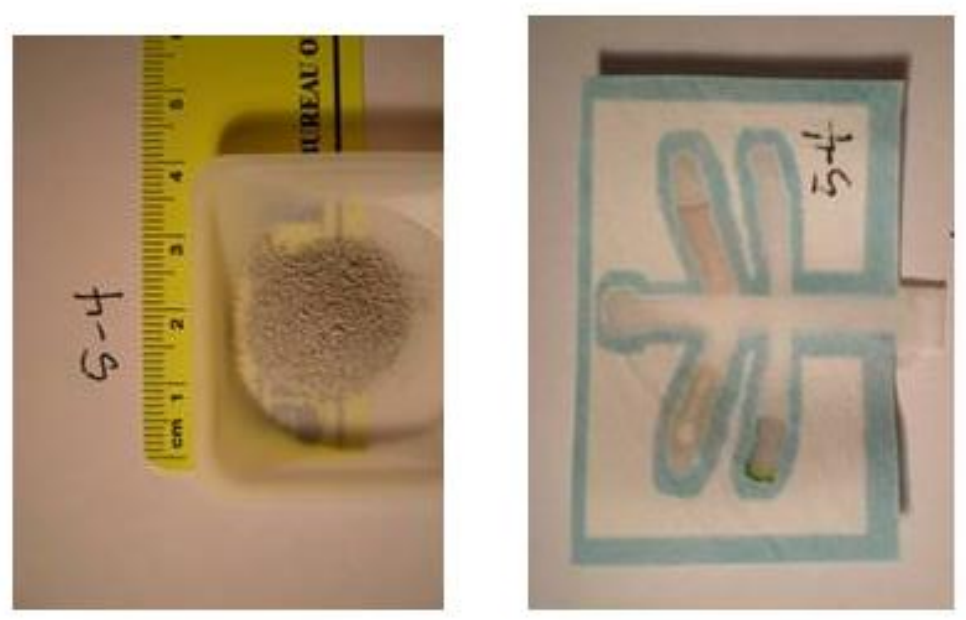

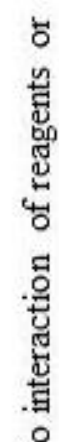
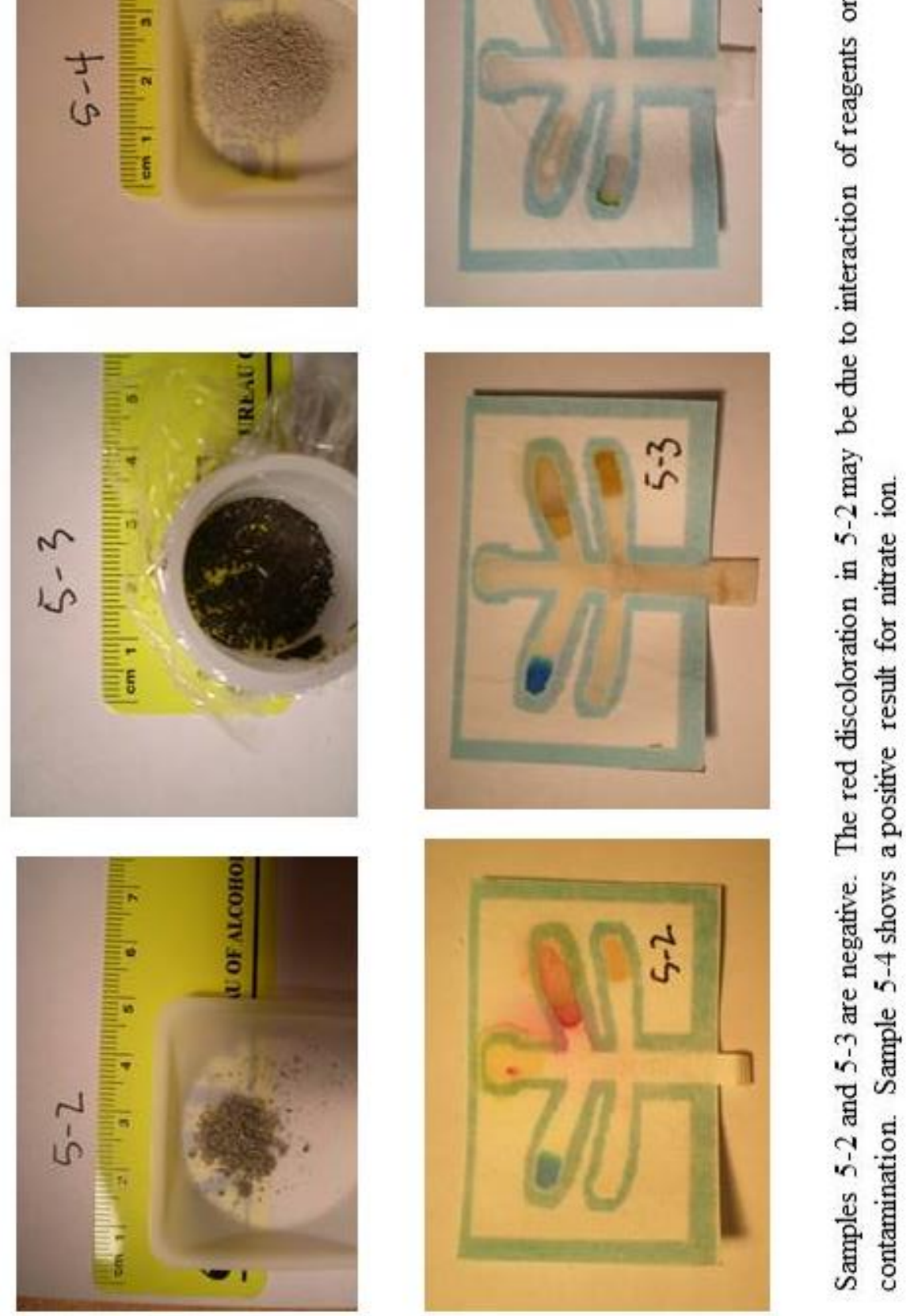
VITA

\title{
INGE CORBIN
}

\author{
$1996-2000$ \\ B.S., Chemistry \\ Metropolitan State College of Denver \\ Denver, CO \\ $2001-2012$ \\ Criminalist \\ Miami-Dade Police Department Crime Laboratory \\ Miami, FL \\ 2012 - present \\ Chemist \\ Defense Forensic Science Center \\ Atlanta, GA

\section{PUBLICATIONS AND PATENTS}

Corbin, I., McCord, B. Detection of the improvised explosives ammonium nitrate (AN) and urea nitrate (UN) using non-aqueous solvents with electrospray ionization and MS/MS detection. Talanta 115 (2013): 533 - 539.

De Perre, C., Corbin, I., Blas, M., McCord, B. Separation and identification of smokeless gunpowder additives by capillary electrochromatography. Journal of Chromatography A 1267 (2012) $259-265$.

Lai H., Corbin I., Almirall J. R. 2008. Headspace sampling and detection of cocaine, MDMA, and marijuana via volatile markers in the presence of potential interferences by solid phase microextraction-ion mobility spectrometry (SPME-IMS). Analytical and Bioanalytical Chemistry 392(1-2): 105 - 113.

McCord B., Corbin I., Bender E. Chromatography of Explosives. In Forensic Investigation of Explosions, Second Edition; Beveridge A., Ed.; International Forensic Science and Investigation Series; CRC Press: Boca Raton, FL, 2011; 585-620.

McCord, Bruce; Corbin, Inge; Blanes, Lucas. 2016. Paper microfluidic devices for the detection of improvised explosives. US 2016/0139102, filed March 15, 2013 and issued May 19, 2016.

Peters, K., Corbin, I., Kaufman, L.M., Zreibe, K.,Blanes, L., McCord, B.R. 2015. Simultaneous colorimetric detection of improvised explosive compounds using microfluidic paper-based analytical devices ( $\mu$ PADs). Analytical Methods 7 (1): 63 - 70. 